The copyright of this thesis vests in the author. No quotation from it or information derived from it is to be published without full acknowledgement of the source. The thesis is to be used for private study or noncommercial research purposes only.

Published by the University of Cape Town (UCT) in terms of the non-exclusive license granted to UCT by the author. 
Department of Mathematics and Applied Mathematics

\title{
DW COMPLEXES AND THEIR UNDERLYING TOPOLOGICAL SPACES
}

\author{
by
}

Patrice Pungu Ntumba

A thesis prepared under the supervision of Prof. P.F. Cherenack for the degree of Doctor of Philosophy in Mathematics

February 2001 
Dédié à ma femme Tshianda et à mon fils Nabii. 


\section{ACKNOWLEDGEMENTS}

I thank my supervisor, Prof. P.F. Cherenack, for his help and encouragement during the course of the thesis. He took an interest in my work, read and criticized with great care various drafts of different parts of the thesis. I gratefully acknowledge his conduct, in the sense that he allowed me to freely decide on the tc pics to study and to shape the thesis to my taste.

I thank Dr J.J. Vermeulen for a great deal of very stimulating exchange of ideas.

I thank Prof. K. Hardie for very encouraging comments and suggestions for future work.

Finally, I thank Prof. B. Hahn, head of our Department of Mathematics and Applied Mathematics for an appointment as a part-time assistant lecturer for the first half of year 2001 . 


\section{Contents}

1 DIFFERENTIAL CW COMPLEXES 11

1.1 Preliminaries . . . . . . . . . . . . . . . . 11

1.2 DW complexes . . . . . . . . . . . . . 31

2 ALTERNATIVE DESCRIPTION OF A DW COMPLEX 78

2.1 Building up a DW complex . . . . . . . . . . . 79

2.2 FW complexes. . . . . . . . . . . . . . . 100

$\begin{array}{ll}\text { BIBLIOGRAPHY } & 125\end{array}$ 


\section{INTRODUCTION}

The naive concept of a DW complex is that of a differential space that can be built up from cells and whose differential structure is defined in terms of differential structures on euclidean unit closed balls. This concept stems from an analogue in the category of topological spaces: the so-called CW complex (introduced by J.H.C. Whitehead in 1949). See [24]. Roughly speaking, a CW complex, as defined in [13], is built up by the successive adjunction of cells of dimensions $1,2,3, \ldots$ The book by Lundell and Weingram [11] is a good reference for an in-depth exposition of $\mathrm{CW}$ complexes. Other references are Bredon [1], Cooke and Finney [5], Maunder [14], Munkres [17], Rotman $[18],,, \ldots$ The "C" in "CW" stands for "closure finite" and the "W" stands for "weak topology".

We adopted the terminology "DW" because of similarities between DW complexes and CW complexes. The "D" in "DW" refers to the category of differential spaces, but "W" means the same type of thing it means in "CW". In fact, we will show in the course of our work that the topology of a DW complex is a weak topology, determined by the closures of cells. For the same 
reason, analogues of $\mathrm{CW}$ complexes in the category of Frölicher spaces are called FW complexes. See [7] or [10] for Frölicher spaces. The category of Frölicher spaces is a full subcategory of the category of differential spaces [3]. For categories, see [12].

This dissertation goes as far as describing DW complexes and their topological properties. Our original aim was to set up cellular homology theory for DW complexes, but we got caught up along the way by our curiousity about the topological properties of DW complexes. This investigation has revealed many nice properties that one would expect DW complexes to have as analogues of $\mathrm{CW}$ complexes. We expanded this inquiry to $\mathrm{FW}$ complexes as well.

It is known that $\mathrm{CW}$ complexes can be defined in two different equivalent ways. But this is not always the case with DW complexes. DW complexes can be described in two different equivalent ways provided they are made up of finitely many cells. The intrinsic (or axiomatic) definition of DW complexes reads like this. Assume that a differential space $(X, \mathcal{F})$ has a DW complex structure. Then the underlying set $X$ is a disjoint union of cells $e$, and if $\bar{e}$ is the closure of $e$ with respect to the topology induced by the functions $\mathcal{F}$, then $\bar{e}$ is the image of some unit closed ball $D^{k}$ under a map $\Phi_{e}$ and is contained in a union of finitely many cells of lesser dimension. The closed ball $D^{k}$ is viewed as a differential subspace of the euclidean differential space $\left(\mathbb{R}^{k}, \mathcal{F}\left(\mathbb{R}^{k}\right)\right)$, and the map $\Phi_{e}$, attached to the cell $e$, is a quotient map. So a function $f: \bar{e} \rightarrow \mathbb{R}$ is smooth on $\bar{e}$ if and only if $f \circ \Phi_{e}: D^{k} \rightarrow \mathbb{R}$ is smooth on $D^{k}$. In this definition, we also want that the differential structure that 
$e$ inherits as a subset of $\bar{e}$ be diffeomorphic to the differential structure on the interior of $D^{k}$. Finally, a function $f: X \rightarrow \mathbb{R}$ is in $\mathcal{F}$ if and only if $\left.f\right|_{\bar{e}}: \bar{e} \rightarrow \mathbb{R}$ is smooth for every $e \in E$.

FW complexes are defined pretty much like DW complexes. The only difference is that balls and cells are regarded as Frölicher spaces. But the fact that on one hand balls have, as Frölicher spaces, the same functions that they have as differential spaces and on the other hand quotients in Frölicher spaces are the same as quotients in differential spaces leads to the result that underlying differential spaces of FW complexes are DW complexes and every Frölicher space whose associated differential space is a DW complex is a FW complex.

From now onward, by underlying space of a differential space we mean the under lying topological space. Likewise, underlying space of a Frölicher space means the actual underlying topological space of the Frölicher space at hand.

As for the alternative description of DW complexes, we introduce the notion of handiness of a differential space. More precisely, a handy differential space is a differential space having the property that given any open covering there is a smooth partition of unity subordinate to that covering. Finite DW complexes can be described as adjunctions of differential spaces. Given an adjunction $X \sqcup_{f} Y$ of differential spaces, for which $f: A \rightarrow Y$ is smooth, we will assume that $X$ is a handy differential space and $A$ is a non-empty closed subspace of $X$. In some instances, we will need $X$ to be a handy Hausdorff differential space, $Y$ a Hausdorff space and $A$ a compact and closed subset 
of $X$. In such instances, the underlying space of $X \sqcup_{f} Y$ is an adjunction of $X$ and $Y$ in topological spaces. This result is pivotal when showing in an alternative way that the underlying space of a finite DW complex is a CW complex.

A survey of the contents now follows.

Chapter 1 deals with the intrinsic definition of DW complexes.

Section 1.1 recalls the notion of CW complexes. We prove that every CW complex in the sense of Rotman is a CW complex in the sense of Fomenko, and every Hausdorff CW complex in the sense of Fomenko is a CW complex in the sense of Rotman. Because of this result, we feel that it is not necessary to define on one side DW complexes à la Rotman and on another side DW complexes à la fomenko.

We then expound on coproducts of differential spaces, showing at one stage that the underlying topology of a coproduct of differential spaces is a coproduct of underlying topological spaces.

Section 1.1 closes with quotient maps in the category of differential spaces. Many results for quotient maps in the category of topological spaces hold as well for quotient maps in differential spaces.

Section 1.2 is the main part of our thesis. This is where we define DW complexes and study their topologies. The main result of this section is that the underlying space of a DW complex is a CW complex. This result 
has many implications. The situation with regard to quotients is rather more complicated, because in general, given a DW complex $X$ and $Y$ a subcomplex, if $\nu: X \rightarrow X / Y$ is the quotient map in differential spaces, the subspace $X-Y$ is not always diffeomorphic to the subspace $X / Y-\{*\}$, where $*=\nu(Y)$. However, if $X$ is assumed to be a handy DW complex and whose topology is regular and $Y$ is taken to be a compact subcomplex, then one gets $X-Y$ diffeomorphic to $X / Y-\{*\}$. And under the above-mentioned assumptions, the fact that $X-Y$ is isomorphic to $X / Y-\{*\}$ clears the way to proving that $X / Y$ is a $\mathrm{DW}$ complex. We appeal to this result later in Chapter 2 when we prove that the underlying space of a FW complex is a DW complex.

Chapter 2 consists of the alternative definition of a DW complex. Unlike CW complexes, only finite DW complexes, meaning DW complexes having a finite number of cells, can be described directly in terms of attaching cells.

Section 2.1 shows how to construct a DW complex from its cells. We start by defining the notion of adjunction of two differential spaces. Given an adjunction $X \sqcup_{f} Y$ of differential spaces $X$ and $Y$; since it is a quotient space, it is therefore unique up to an isomorphism. The most important problem treated here is that, given a handy differential space $X$ whose topology is Hausdorff and $A$ a compact closed subspace of $X$, if $f: A \rightarrow Y$ carries $A$ smoothly into a Hausdorff differential space $Y$, then the adjunction $X \sqcup_{f} Y$, taken in differential spaces, acquires the quotient topology from $X \sqcup Y$. We then examine the particular case whereby $X$ is a coproduct of finitely many unit balls, taken in differential spaces, and $A$ is the coproduct of boundaries 
of balls and $Y$ is a DW complex. Since $X$ is finite, it follows that $A$ is a compact closed subset of $X$.

In section 2.2, we explore the class of FW complexes. Theorem 2.1 [4] is needed to show that every closed ball has, as a differential space, the same functions that it has as a Frölicher space. This result, in turn, is instrumental when it is necessary to show that, given a Frölicher space $(X, \mathcal{F}, \mathcal{C})$, if $(X, \mathcal{F})$ is a DW complex, then $(X, \mathcal{F}, \mathcal{C})$ is a FW complex. On another side, if $(X, \mathcal{F}, \mathcal{C})$ is a FW complex, then $(X, \mathcal{F})$ is a DW complex. 


\section{Chapter 1}

\section{DIFFERENTIAL CW}

\section{COMPLEXES}

\subsection{Preliminaries}

This section recalls the notion of CW complex as generally defined in algebraic topology, and construction of coproducts in the category of differential spaces. One might like to define CW complexes as in [18] or as in [6], but we show that these two definitions are equivalent.

Definition [18] A CW complex in the sense of Rotman is a triple $(X, E, \Phi)$, where $X$ is a Hausdorff space, $E$ is a family of disjoint subspaces of $X$, and $\Phi=\left\{\Phi_{e}: e \in E\right\}$ is a family of maps satisfying the following conditions: 
(1R) $X=\cup\{e: e \in E\}$;

(2R) If $D^{k}$ is the unit $k$-dimensional closed ball ( the boundary of $D^{k}$ is the unit $(k-1)$-dimensional sphere $\left.S^{k-1}\right)$, and if $X^{(k)}$ is the union of all cells in $X$ of dimension $\leq k$ then the map

$$
\Phi_{e}:\left(D^{k}, S^{k-1}\right) \rightarrow\left(e \cup X^{(k-1)}, X^{(k-1)}\right)
$$

is a relative homeomorphism, meaning that $\Phi_{e}: D^{k} \rightarrow e \cup X^{(k-1)}$ is a continuous map and its restriction $\left.\Phi_{e}\right|_{D^{k}-S^{k-1}}$ carries the open ball $D^{k}-S^{k-1}$ homeomorphically onto $e$;

(3R) If $e \in E$, then its closure $\bar{e}$ is contained in a finite union of cells in $E$;

(4R) $X$ has the weak topology determined by the collection $\{\bar{e}: e \in E\}$, that is a subset $A$ of $X$ is closed if and only if $A \cap \bar{e}$ is closed in $\bar{e}$ for all $e \in E$.

The maps $\Phi_{e}$ are called the characteristic maps for the CW complex $X$, and the subspaces $e \in E$ are the $k$-cells of $X$. If the subspace $e$ of $X$ is a $k$-cell,

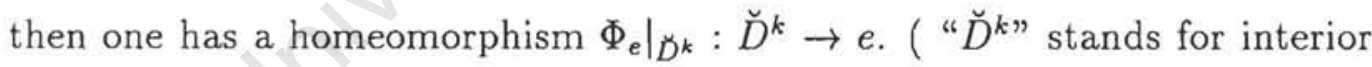
of the unit closed ball $D^{k}$.)

The subspace $X^{(k)}, k \geq 0$, defined in axiom (2R) is called the $k$-skeleton of $X$. It is a CW complex on its own right. See Example 8.21 in [18].

As a precursor to the differential case, it shall be proved that the notion of $\mathrm{CW}$ complex in the sense of Rotman is the same as that in the sense of 
Fomenko, provided the topological space in Fomenko's definition is Hausdorff. Now, let us define the notion of $\mathrm{CW}$ complex according to Fomenko [6].

Definition A topological space $X$ is a CW complex in the sense of Fomenko if

(1F) $X=\cup\{e: e \in E\}$, where as above $E$ is a family of pairwise disjoint subsets of $X$, called cells ;

$(2 \mathrm{~F})$ For each $k$-cell $e$, there is a continuous map $\Psi_{e}: D^{k} \rightarrow X$ of the closed $k$-dimensional ball $D^{k}$ into the space $X$ such that $\left.\Psi_{e}\right|_{D^{k}}$ is a homeomorphism onto the cell $e$;

(3F) For each cell $e$ of $X$, the subset $\partial e:=\bar{e}-e$ is contained in a finite union of cells of lesser dimensions;

(4F) If $e$ is a $k$-cell in $X$, a subset $W \subset X$ is closed if and only if the pullback $\Psi_{e}^{-1}(W) \subset D^{k}$ is closed in $D^{k}$.

In the context of Fomenko, by characteristic maps we mean maps $\Psi_{e}$. We will call $\partial e$ the boundary of the cell $e$, a terminology borrowed from [6], [13] Note that $\partial e$ need not coincide with the boundary of $e$ in the sense of point set topology.

The following lemma shows how characteristic maps $\Phi_{e}$ and $\Psi_{e}$ relate.

Lemma 1.1.1 If $(X, E)$ is a $C W$ complex both in the sense of Rotman and in the sense of Fomenko, where the characteristic maps are given by sets 
$\Phi=\left\{\Phi_{e}: e \in E\right\}$ and $\Psi=\left\{\Psi_{e}: e \in E\right\}$ for the Rotman structure and Fomenko structure respectively, then for each $k$-cell $e \in E$, one has

$$
\Phi_{e}\left(D^{k}\right)=\bar{e}=\Psi_{e}\left(D^{k}\right) .
$$

Proof. For the equation $\Phi_{e}\left(D^{k}\right)=\bar{e}$, one may refer to [18]. The same argument holds for the equation $\Psi_{e}\left(D^{k}\right)=\bar{e}$. Indeed, since $D^{k}=\overline{D^{k}-S^{k-1}}$ and $\Psi_{e}$ is continous, one has

$$
\Psi_{e}\left(D^{k}\right)=\Psi_{e}\left(\overline{\breve{D}^{k}}\right) \subset \overline{\Psi_{e}\left(\breve{D}^{k}\right)}=\bar{e}
$$

On the other hand, the image $\Psi_{e}\left(D^{k}\right)$ is compact and since $X$ is Hausdorff, $\Psi_{e}\left(D^{k}\right)$ is a closed subset of $X$. But $e=\Psi_{e}\left(\breve{D}^{k}\right) \subset \Psi_{e}\left(D^{k}\right)$, therefore $\bar{e} \subset \overline{\Psi_{e}\left(D^{k}\right)}=\Psi_{e}\left(D^{k}\right)$.

Lemma 1.1.2 Every $C W$ complex in the sense of Rotman is a CW complex in the sense of Fomenko.

Proof. Let $(X, E, \Phi)$ be a CW complex in the sense of Rotman. We claim that $(E, \Phi)$ defines a Fomenko CW structure on the space $X$.

Let us verify the Fomenko axioms.

That $(1 \mathrm{~F})$ and $(2 \mathrm{~F})$ hold is immediate. To get $(3 \mathrm{~F})$, suppose $e \in E$ is a $k$-cell. By axiom $(2 \mathrm{R}), \Phi_{e}\left(S^{k-1}\right) \subset X^{(k-1)}$; since $X^{(k-1)}$ and $e$ are disjoint, it follows that $\Phi_{e}\left(S^{k-1}\right) \cap e=\emptyset$. Moreover, as $\Phi_{e}\left(D^{k}-S^{k-1}\right)=e$ one has 
$\Phi_{e}\left(D^{k}\right)=e \cup \Phi_{e}\left(S^{k-1}\right)$, a disjoint union. But $\Phi_{e}\left(D^{k}\right)=\bar{e}$ by Lemma 1 above, therefore $\partial e=\Phi_{e}\left(S^{k-1}\right) \subset X^{(k-1)}$. Thus the boundary $\partial e$ cannot contain cells of dimension $\geq k$. On account of $(3 \mathrm{R}), \bar{e}$ is contained in a finite union of cells of $E$. Thus, $\partial e$ is contained in a finite union of cells of dimension $\leq k-1$, and hence $(3 \mathrm{~F})$ holds.

Finally, let $W$ be a closed subset of $X$ for the weak topology generated by the collection $\{\bar{e}: e \in E\}$. For all $e \in E, W \cap \bar{e}$ is closed in $\bar{e}$. Suppose that $e$ is a cell of dimension $k$. As $\Phi_{e}$ is continuous, then $\Phi_{e}^{-1}(W)=\Phi_{e}^{-1}(W \cap \bar{e})$ is closed in $D^{k}$. Conversely, assume that $W$ is a subset of $X$ such that $\Phi_{e}^{-1}(W \cap \bar{e})$ is a closed set for each cell $e$. If $k(e)$ indicates the dimension of a cell $e$ of $X$, let $\varphi: \sqcup_{e \in E} D^{k(e)} \rightarrow X$ be the map $\varphi=\sqcup_{e} \Phi_{e}$; one can prove, see [18], that $\varphi$ is anidentification. Thus, for each $e \in E, \Phi_{e}$ is anidentification. It follows that if $\Phi_{e}^{-1}(W \cap \bar{e})$ is closedin $D^{k}$ then $W \cap \bar{e}$ is a closed subset of $\bar{e}$; therefore $W$ is a closed subset of $X$.

Conversely, we have

Lemma 1.1.3 A Hausdorff $C W$ complex in the sense of Fomenko can be viewed as a CW complex in the sense of Rotman.

Proof. Let $(X, E, \Psi)$ be a Hausdorff CW complex in the sense of Fomenko. It is easy matter to show that (1R) and (3R) hold.

As for each $k$-cell $e, \Psi_{e}\left(D^{k}\right)=\bar{e}$, and $\partial e \subset X^{(k-1)}$ by axiom $(3 F)$; it follows that $\Psi_{e}\left(D^{k}\right) \subset e \cup X^{(k-1)}$. Now, let $x \in S^{k-1}$. Then there is a 
sequence $\left(x_{n}\right)_{n \in \mathbb{N}}$ in $D^{k}-S^{k-1}$ that converges to $x$. Since for each cell $e, \Psi_{e}$ is continuous, it is clear that $\left(\Psi_{e}\left(x_{n}\right)\right)_{n \in \mathbb{N}}$ is a set of points in the cell $e$ converging to the point $\Psi_{e}(x) \in \bar{e}$. Suppose that $\Psi_{e}(x) \notin \partial e$; thus $\Psi_{e}(x) \in e$. Since $\left.\Psi_{e}\right|_{D^{k}-S^{k-1}}$ is a homeomorphism onto $e$, the sequence $\left(x_{n}\right)$ converges to a point $y \in D^{k}-S^{k-1}$. But $D^{k}$ is Hausdorff, therefore $x=y$; and thus $x \notin S^{k-1}$. This contradiction implies that $\Psi_{e}(x) \in \partial e$, and hence $\Psi_{e}\left(S^{k-1}\right) \subset \partial e$. Moreover, since $\partial e \subset X^{(k-1)}$, it follows that $\Psi_{e}\left(S^{k-1}\right) \subset X^{(k-1)}$. We gather from the preceding argument that the map

$$
\Psi_{e}:\left(D^{k}, S^{k-1}\right) \rightarrow\left(e \cup X^{(k-1)}, X^{(k-1)}\right)
$$

is a relative homeomorphism; hence (2R) holds.

Finally, we need to show that if $V$ is a closed subset of $X$ according to (4F), then $V$ is a closed set for the weak topology determined by the collection $\{\bar{e}: e \in E\}$. First note that for each cell $e, V \cap \bar{e}=\Psi_{e}\left(\Psi_{e}^{-1}(V \cap \bar{e})\right)$. But $D^{k}$ is compact; since $(4 \mathrm{~F})$ says that $\Psi_{e}^{-1}(V \cap \bar{e})=\Psi_{e}^{-1}(V)$ is closed in $D^{k}$, it follows that $\Psi_{e}^{-1}(V \cap \bar{e})$ is compact. Thus, $V \cap \bar{e}$ is compact in $\bar{e}$; and as a subset of a Hausdorff space, $V \cap \bar{e}$ must be closed in $\bar{e}$.

It follows from Lemmas 1.1.2 and 1.1.3 above that:

1. In the category of Hausdorff spaces, the Rotman CW complex structure is the same as the Fomenko CW complex structure. Thus, in Hausdorff spaces, one need not specify whether a CW complex is Rotman or Fomenko.

2. The topology of a CW complex is equivalently described by either (4R) 
or $(4 \mathrm{~F})$.

Our next goal is to introduce analogues of $\mathrm{CW}$ complexes in the category of differential spaces. We shall call them differential CW complexes or, simply, DW complexes. But first, we define the notion of differential space and en passant present some useful results on coproducts of differential spaces. As references for differential spaces, see [22], [23] .

Definition Let $X$ be any set, $\mathcal{F}$ be a set of real-valued functions on $X$, and $\mathcal{T}$ be the weakest topology on $X$ for which all functions in $\mathcal{F}$ are continuous. The pair $(X, \mathcal{F})$ is called a differential space if and only if:

(i) any function $g: X \rightarrow \mathbb{R}$ such that for any point $p \in X$ there exists an open neighbourhood $U \in \mathcal{T}$ of $p$ such that $\left.g\right|_{U}=\left.f\right|_{U}$ for some $f \in \mathcal{F}$ is also in $\mathcal{F}$.

(ii) given $f_{1}, \ldots, f_{n} \in \mathcal{F}$, if $\omega: \mathbb{R}^{n} \rightarrow \mathbb{R}$ is a smooth real-valued function defined on $\mathbb{R}^{n}$ then $\omega \circ\left(f_{1}, \ldots, f_{n}\right) \in \mathcal{F}$.

We shall say that $\mathcal{F}$ is closed with respect to localization provided axiom (i) is fulfilled, and $\mathcal{F}$ is closed with respect to composition with smooth functions provided axiom (ii) is satisfied.

If $(X, \mathcal{F})$ is a differential space, the family $\mathcal{F}$ is called a differential (or smooth) structure; any function $f$ of $\mathcal{F}$ is called a differential (or smooth) map. For any two differential spaces $(X, \mathcal{F})$ and $(Y, \mathcal{G})$, a mapping $f: X \rightarrow Y$ 
is called a smooth map if $\alpha \circ f \in \mathcal{F}$ for all $\alpha \in \mathcal{G}$. Differential spaces and smooth maps constitute a category. We shall denote it by $\mathcal{D I F \mathcal { F }}$ as in [3].

Lemma 1.1.4 Let $X$ be any set, let $\mathcal{F}_{0}$ be a family of real-valued functions on $X$ and let $\mathcal{T}_{0}$ be the initial topology induced by the functions of $\mathcal{F}_{0}$ on $X$. Then the family $\mathcal{F}$ of functions $f: X \rightarrow \mathbb{R}$ such that if $f \in \mathcal{F}$ then, given any point $p \in X$, there are functions $f_{1}, \ldots, f_{n} \in \mathcal{F}_{0}, \omega \in C^{\infty}\left(\mathbb{R}^{n}\right)$ and an open neighbourhood $U \in \mathcal{T}_{0}$ of $p$ such that

$$
\left.f\right|_{U}=\left.\omega \circ\left(f_{1}, \ldots, f_{n}\right)\right|_{U}
$$

is the smallest differential structure, containing $\mathcal{F}_{0}$, on $X$.

Proof. If $\mathcal{T}$ is the topology induced by the functions in $\mathcal{F}$, it is easy to see that $\mathcal{T}=\mathcal{T}_{0}$.

The family $\mathcal{F}$ is closed with respect to localization. Indeed, let $g: X \rightarrow \mathbb{R}$ be a function such that for any point $p$ there exists a function $f \in \mathcal{F}$ such that $\left.g\right|_{U}=\left.f\right|_{U}$, where $U$ is an open neighbourhood of $p$. Since $f$ is a function in $\mathcal{F}$, then by hypothesis one can find an open neighbourhood $V$ of $p$ and functions $f_{1}, \ldots, f_{n} \in \mathcal{F}_{0}$ such that $\left.f\right|_{V}=\left.\omega \circ\left(f_{1}, \ldots, f_{n}\right)\right|_{V}$ for some smooth $\omega \in C^{\infty}\left(\mathbb{R}^{n}\right)$. But $U \cap V$ is an open neighbourhood of $p$, and one has

$$
\left.g\right|_{U \cap V}=\left.\omega \circ\left(f_{1}, \ldots, f_{n}\right)\right|_{U \cap V} .
$$

Thus, $g \in \mathcal{F}$ and hence $\mathcal{F}$ is closed with respect to localization.

The next axiom requires that compositions with smooth functions $\omega \in C^{\infty}\left(\mathbb{R}^{n}\right)$ be also functions of $\mathcal{F}$. So consider $g_{1}, \ldots, g_{n} \in \mathcal{F}$ and $\omega \in C^{\infty}\left(\mathbb{R}^{n}\right)$. By 
hypothesis, for every point $p \in X$, there exist open neighbourhoods $U_{i}$ of $p$, $i=1, \ldots, n$, such that $\left.g_{i}\right|_{U_{i}}=\omega_{i} \circ\left(f_{i_{1}}, \ldots, f_{i_{m}}\right)$, where $f_{i_{1}}, \ldots, f_{i_{m}} \in \mathcal{F}_{0}$ and $\omega_{i} \in C^{\infty}\left(\mathbb{R}^{i_{m}}\right)$. It follows that

$$
\begin{aligned}
\omega \circ & \left.\left(g_{1}, \ldots, g_{n}\right)\right|_{n_{i=1}^{n} U_{i}}= \\
& \left.\omega \circ\left(\omega_{1}, \ldots, \omega_{n}\right)\left(f_{1_{1}}, \ldots, f_{1_{m}}, \ldots, f_{n_{1}}, \ldots, f_{n_{m}}\right)\right|_{n_{i=1}^{n} U_{i}} .
\end{aligned}
$$

Since $f_{1_{1}}, \ldots, f_{n_{m}} \in \mathcal{F}_{0}$ and $\omega \circ\left(\omega_{1}, \ldots, \omega_{n}\right) \in C^{\infty}\left(\mathbb{R}^{l}\right)$, where $l=\sum_{i=1}^{n} i_{m}$, it follows that $\omega \circ\left(g_{1}, \ldots, g_{n}\right) \in \mathcal{F}$.

Now suppose that there exists another differential structure $\mathcal{G}$ such that $\mathcal{F}_{0} \subset \mathcal{G}$. Let $f \in \mathcal{F}$, and $p$ be any point of $X$. By hypothesis, for some $f_{1}, \ldots, f_{n} \in \mathcal{F}_{0}, \omega \in C^{\infty}\left(\mathbb{R}^{n}\right)$, one has $\left.f\right|_{U}=\left.\omega \circ\left(f_{1}, \ldots, f_{n}\right)\right|_{U}$ where $U$ is an open neighbourhood of $p$. Since $\mathcal{G}$ is a differential structure and $\mathcal{F}_{0} \subset \mathcal{G}$, the function $\omega \circ\left(f_{1}, \ldots, f_{n}\right)$ belongs to $\mathcal{G}$. Thus $f \in \mathcal{G}$ and hence $\mathcal{F} \subset \mathcal{G}$.

The family $\mathcal{F}_{0}$ is said to generate the differential structure $\mathcal{F}$. We denote by $\operatorname{gen}_{X}\left(\mathcal{F}_{0}\right)$ or simply by gen $\left(\mathcal{F}_{0}\right)$ the differential structure generated by the set $\mathcal{F}_{0}$ of real-valued functions on $X$.

Lemma 1.1.5 Let $(X, \mathcal{F})$ a differential space, and let $(Y, \mathcal{G})$ be another differential space whose differential structure $\mathcal{G}$ is the structure generated by some family $\mathcal{H} \subset \mathbb{R}^{Y}$. A function $\varphi:(X, \mathcal{F}) \rightarrow(Y, \mathcal{G})$ is smooth if and only if $f \circ \varphi \in \mathcal{F}$ for sach $f \in \mathcal{H}$.

Proof. Suppose that $\varphi$ is smooth. Since $\mathcal{H} \subset \mathcal{G}$, it follows that $f \circ \varphi \in \mathcal{F}$ for each $f \in \mathcal{H}$. 
Now suppose that $f \circ \varphi \in \mathcal{F}$ for each $f \in \mathcal{H}$. Let $g \in \mathcal{G}$. If $p$ is a point in $Y$, then there exist $n$ functions $f_{1}, \ldots, f_{n} \in \mathcal{H}$ and a smooth function $\omega: \mathbb{R}^{n} \rightarrow \mathbb{R}$ such that

$$
\left.g\right|_{U}=\left.\omega \circ\left(f_{1}, \ldots, f_{n}\right)\right|_{U}
$$

for some open neighbourhood $U$ of $p$. Since the underlying topology of $\mathcal{G}$ is the topology induced by the functions of $\mathcal{H}$, one can choose $h \in \mathcal{H}$ and an open subset $W \subset \mathbb{R}$ such that $p \in h^{-1}(W) \subset U$. So, on $h^{-1}(W)$, one also has

$$
\left.g\right|_{h^{-1}(W)}=\left.\omega \circ\left(f_{1}, \ldots, f_{n}\right)\right|_{h^{-1}(W)} .
$$

But $h \circ \varphi \in \mathcal{F}$, therefore $(h \circ \varphi)^{-1}(W)$ is an open neighbourhood of any $u \in X$ such that $p=\varphi(u)$. And moreover, we have

$$
\left.g \circ \varphi\right|_{h^{-1}(W)}=\left.\omega \circ\left(f_{1} \circ \varphi, \ldots, f_{n} \circ \varphi\right)\right|_{h^{-1}(W)} .
$$

Since $f_{i} \circ \varphi \in \mathcal{F}$, for each $i=1, \ldots, n$, and since $\omega \circ\left(f_{1} \circ \varphi, \ldots, f_{n} \circ \varphi\right) \in \mathcal{F}$, it follows that $g \circ \varphi$ is locally smooth. Thus $g \circ \varphi \in \mathcal{F}$, and hence $\varphi$ is smooth.

Definition [22] Let $(X, \mathcal{F})$ and $(Y, \mathcal{G})$ be differential spaces. A function $\varphi:(X, \mathcal{F}) \rightarrow(Y, \mathcal{G})$ is called a diffeomorphism if the set $\operatorname{map} \varphi: X \rightarrow Y$ is a bijection and if $\varphi$ and $\varphi^{-1}$ are both smooth.

Lemma 1.1.6 Let $\varphi:(X, \mathcal{F}) \rightarrow(Y, \mathcal{G})$ be a diffeomorphism of differential spaces, let $\left(A, \mathcal{F}_{A}\right)$ be a subspace of $(X, \mathcal{F})$ and let $\left(B, \mathcal{G}_{B}\right)$ be a subspace of $(Y, \mathcal{G})$, where $B=\varphi(A)$. Then $\left.\varphi\right|_{A}:\left(A, \mathcal{F}_{A}\right) \rightarrow\left(B, \mathcal{G}_{B}\right)$ is a diffeomorphism. 
Proof. Let $f \in \mathcal{G}_{B}$ be such that $f=\left.h\right|_{B}$, where $h \in \mathcal{G}$. Then

$$
\left.f \circ \varphi\right|_{A}=\left.\left.(h \circ \varphi)\right|_{A} \in \mathcal{F}\right|_{A}
$$

By virtue of Lemma 1.1.5, it follows that $\left.\varphi\right|_{A}$ is smooth.

Now let $k \in \mathcal{F}_{A}$ be such that $k=\left.l\right|_{A}$, with $l \in \mathcal{F}$. Since $\varphi$ is a bijection, then

$$
\left(\left.\varphi\right|_{A}\right)^{-1}=\left.\varphi^{-1}\right|_{B}
$$

Thus,

$$
k \circ\left(\left.\varphi\right|_{A}\right)^{-1}=\left.\left(l \circ \varphi^{-1}\right)\right|_{B} \in \mathcal{G}_{B}
$$

Hence, by Lemma 1.1.5, $\left(\left.\varphi\right|_{A}\right)^{-1}$ is smooth, and the proof is finished.

Lemma 1.1.7 Let $(X, \mathcal{F})$ be a differential space, and let $p$ be any point in $X$. If $U$ is an open neighbourhood of $p$, then there exists a nonnegative function $h \in \mathcal{F}$ such that $p \in h^{-1}(0, \infty) \subset U$.

Proof. The hypothesis that $U$ is an open neighbourhood $p$ ensures that there exists a function $f \in \mathcal{F}$ such that $p \in f^{-1}(a, b) \subset U$, where $(a, b)$ is an open interval. Now suppose that $\alpha: \mathbb{R} \rightarrow[0, \infty)$ is a smooth function such that $f(p) \in \alpha^{-1}(0, \infty) \subset(a, b)$. As $\mathcal{F}$ is closed under composition with smooth functions, it follows that $\alpha \circ f \in \mathcal{F}$; thus setting $h=\alpha \circ f$, one obtains a nonnegative function $h \in \mathcal{F}$ such that $p \in h^{-1}(0, \infty) \subset U$.

Definition Let $\left\{\left(X_{j}, \mathcal{F}_{j}\right) \mid j \in J\right\}$ be a family of differential spaces, let $X$ be an arbitrary set and let $\left\{f_{j}: X \rightarrow X_{j} \mid j \in J\right\}$ be a family of functions. 
A differential structure $\mathcal{F}$ on $X$ is said to be induced on $X$ from differential spaces $\left\{\left(X_{j}, \mathcal{F}_{j} \mid j \in J\right\}\right.$ if

$$
\mathcal{F}=\operatorname{gen}\left(\left\{\alpha_{j} \circ f_{j} \mid \alpha_{j} \in \mathcal{F}_{j}, j \in J\right\}\right) .
$$

Analogously, a differential structure $\mathcal{G}$ is said to be coinduced on a set $Y$ from the family $\left\{\left(X_{j}, \mathcal{F}_{j}\right) \mid j \in J\right\}$ of differential spaces by a family of functions $\left\{g_{j}: X_{j} \rightarrow Y \mid j \in J\right\}$ if

$$
\mathcal{G}=\left\{\alpha \in \mathbb{R}^{Y} \mid \alpha \circ g_{j} \in \mathcal{F}_{j}, j \in J\right\} .
$$

We show in Lemma 1.2.1 that coinduced structures exist. One may refer to [15] for induced structures. In categorical terms, induced structures are called initial structures and coinduced structures are called final structures. We borrowed the term "coinduced" from Mulatrzynski. See [16]

We will adopt the following notation. Suppose that for each $j \in J, X_{j}$ is a differential space. If $\mathcal{F}$ is a structure induced by functions $\left\{f_{j}: X \rightarrow\right.$ $\left.X_{j} \mid j \in J\right\}$ then $\mathcal{F}$ will be denoted by ind $\left(\left\{f_{j}: j \in J\right\}\right)$. However if $\mathcal{G}$ is a structure coinduced by functions $\left\{g_{j}: X_{j} \rightarrow X \mid j \in J\right\}, \mathcal{G}$ will be denoted by $\operatorname{coind}\left(\left\{g_{j}: j \in J\right\}\right)$. An induced differential structure of particular interest is the subspace differential structure. Let $A$ be a subset of $X$, where $(X, \mathcal{F})$ is a differential space. The differential structure induced by $\iota_{A}: A \rightarrow X$, $\iota_{A}(x)=x$ for ail $x \in A$, is called a subspace differential structure. We denote by $\mathcal{F}_{A}$ the subspace structure. Thus, $\mathcal{F}_{A}=\operatorname{gen}\left(\left\{\left.f\right|_{A} ; f \in \mathcal{F}\right\}\right)$. The pair $\left(A, \mathcal{F}_{A}\right)$ is called a differential subspace of $(X, \mathcal{F})$.

Lemma 1.1.8 Let $\left\{\left(X_{j}, \mathcal{F}_{j}\right) ; j \in J\right\}$ be a family of differential spaces, let $X=\bigsqcup_{j \in J} X_{j}$ be a disjoint union of underlying sets $X_{j}$, and let $\mathcal{F} \subset \mathbb{R}^{X}$ be 
such that if $f \in \mathcal{L}$, then $\left.f\right|_{X_{j}} \in \mathcal{F}_{j}$ for each $j \in J$. Then, $\mathcal{F}$ is a differential structure on $X$.

The pair $(X, \mathcal{F})$ is called a coproduct of the family $\left\{\left(X_{j}, \mathcal{F}_{j}\right) \mid j \in J\right\}$.

Proof. Let $\iota_{X}: X_{j} \rightarrow X$ be the inclusion of $X_{j}$ into $X$. It is easy to see that $\mathcal{F}=\operatorname{coind}\left(\left\{\iota_{X}: j \in J\right\}\right)$ and thus $\mathcal{F}$ is a differential structure on $X$.

Now, let $f_{j}:\left(X_{j}, \mathcal{F}_{j}\right) \rightarrow(Y, \mathcal{G})$ be a map of differential spaces and

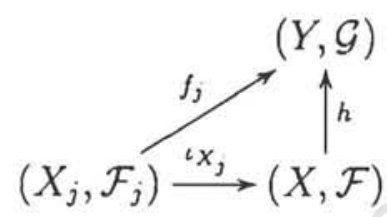

a commutative diagram with $h(x)=f_{j}(x)$ for $x \in X_{j}$. As $h$ is uniquely defined in sets, so is it unique in differential spaces. Furthermore, $h$ is smooth because for each $g \in \mathcal{G}$ one has $\left.g \circ h\right|_{X},=g \circ f_{j} \in \mathcal{F}_{j}$.

Lemma 1.1.9 Let $\left\{\left(X_{j}, \mathcal{F}_{j}\right) \mid j \in J\right\}$ be differential spaces, and let $(X, \mathcal{F})$ be their coproduct. If $\mathcal{T}$ (resp., $\mathcal{T}_{j}$ ) is the underlying topology of $\mathcal{F}$ ( resp., $\left.\mathcal{F}_{j}\right)$, then a subset $V$ of $X_{j}$ is $\mathcal{T}_{j}$-open if and only if $V=T \cap X_{j}$ for some $T \in \mathcal{T}$. In other words, if $\mathcal{T}_{X_{j}}$ is the topology induced from $\mathcal{T}$ on $X_{j}, j \in J$, then $\mathcal{T}_{X}=\mathcal{T}_{j}$.

Proof. Let $T$ be an open set for the topology $\mathcal{T}$. Then for each point $p \in T$, there exists a map $f_{p} \in \mathcal{F}$ such that $p \in f_{p}^{-1}(0, \infty) \subset T$. Thus, $T=\cup_{p \in T} f_{p}^{-1}(0, \infty)$. Since

$$
f_{p}^{-1}(0, \infty) \cap X_{j}=\left(\left.f_{p}\right|_{X}\right)^{-1}(0, \infty) \in \mathcal{T}_{j}
$$


it follows that $T \cap X_{j} \in \mathcal{T}_{j}$. Hence, for every $j \in J, \mathcal{T}_{X_{j}} \subset \mathcal{T}_{j}$.

Conversely, let $V \in \mathcal{T}_{j}$, where $j$ is fixed in $J$. Then for any point $q \in V$, there exists a function $f \in \mathcal{F}_{j}$ such that $q \in f^{-1}(0, \infty) \subset V$. Next, define $g: X \rightarrow \mathbb{R}$ by setting

$$
g(x)=\left\{\begin{array}{ll}
f(x) & \text { for } x \in X_{j} \\
0 & \text { for } x \notin X_{j} .
\end{array},\right.
$$

It is easy to see that

$$
\left.g\right|_{X_{k}}= \begin{cases}f & \text { for } k=j \\ 0 & \text { for } k \neq j .\end{cases}
$$

So, $g \in \mathcal{F}$ and $g^{-1}(0, \infty)=f^{-1}(0, \infty)$. Then $q \in g^{-1}(0, \infty) \subset V$; thus $V$ is an open subset of $X$ for the topology $\mathcal{T}$. Wherefore, $\mathcal{T}_{j} \subset \mathcal{T}_{X}$, for each $j \in J$.

Corollary 1.1.1 Let $\left\{\left(X_{j}, \mathcal{F}_{j}\right) ; j \in J\right\}$ be a family of differential spaces and let $X=\sqcup_{j \in J} X_{j}$ (disjoint union). If $\mathcal{F}$ is the coproduct structure on $X$ coinduced by $\left\{\left(X_{j}, \mathcal{F}_{j}\right) ; j \in J\right\}$, then the $\mathcal{F}$-induced topology $\mathcal{T}$ is the weakest topology on $X$ determined by $\left\{\left(X_{j}, \mathcal{T}_{j}\right) ; j \in J\right\}$, where $\mathcal{T}_{j}$ is the underlying topology of $\mathcal{F}_{j}$, for each $j \in J$.

Proof. It is evident that for each subset $T \subset X, T \in \mathcal{T}$ if and only if $T \cap X_{j} \in \mathcal{T}_{j}$ for each $j \in J$.

Lemma 1.1.10 If $(X, \mathcal{F})$ is a coproduct of differential spaces 
$\left\{\left(X_{j}, \mathcal{F}_{j}\right) ; j \in J\right\}$. and $i f_{X_{X}}$ is the inclusion $X_{j} \rightarrow X$, then

$$
\mathcal{F}_{X_{j}}=\mathcal{F}_{j}
$$

where $\mathcal{F}_{X},=\operatorname{ind}\left(\left\{\iota_{X},\right\}\right)$.

Proof. Let $f \in \mathcal{F}_{X}$. There exists an open covering $\mathcal{U}$ of $X$ (with respect to the underlying topology of $\mathcal{F}$ ) such that, for each $U \in \mathcal{U},\left.f\right|_{U \cap X_{j}}=\left.g_{U}\right|_{U \cap X_{,}}$ for some $g_{U} \in \mathcal{F}$. By Lemma 1.1.9, $U \cap X_{j} \in \mathcal{T}_{j}$ for each $U \in \mathcal{U}$; since $\left.g_{U}\right|_{X} \in \mathcal{F}_{j}$, it follows that $f \in \mathcal{F}_{j}$. Hence $\mathcal{F}_{X}, \subset \mathcal{F}_{j}$. On the other side, let $h \in \mathcal{F}_{j}$ and let $k_{U}: X \rightarrow \mathbb{R}$, where $U \in \mathcal{U}$, be defined by

$$
k_{U}(x)=\left\{\begin{array}{ll}
h(x) & \text { for } x \in X_{j} \\
0 & \text { for } x \in X_{l}, l \neq j
\end{array} .\right.
$$

Since $\left.h\right|_{U \cap X}=\left.k_{U}\right|_{U \cap X}$; so $h \in \mathcal{F}_{X}$, and hence $\mathcal{F}_{j} \subset \mathcal{F}_{X_{j}}$.

Lemma 1.1.11 Let $\left\{\left(X_{j}, \mathcal{F}_{j}\right) ; j \in J\right\}$ be a collection of differential spaces, and let $(X, \mathcal{F})$ be their coproduct space. Then the underlying topological space $(X, \mathcal{T})$ of the differential space $(X, \mathcal{F})$ is the topological coproduct of the family $\left\{\left(X_{j}, \mathcal{T}_{j}\right) ; j \in J\right\}$, where for each $j, \mathcal{T}_{j}$ denotes the topology induced by $\mathcal{F}_{j}$ on $X_{j}$.

Proof. Let $\mathcal{T}^{\prime}$ denote the coproduct topology of topological spaces $\left\{\left(X_{j}, \mathcal{T}_{j}\right) ; j \in J\right\}$. Since $\mathcal{F}$ is a coproduct structure, then if $f \in \mathcal{F}$ and $U$ is an open subset of $\mathbb{R}$, one has $f^{-1}(U) \cap X_{j}=\left(\left.f\right|_{X_{j}}\right)^{-1}(U) \in \mathcal{T}_{j}$ for each $j \in J$. Therefore, $f^{-1}(U) \in \mathcal{T}^{\prime}$, and hence $\mathcal{T} \subset \mathcal{T}^{\prime}$. 
Now, take $V \in \mathcal{T}^{\prime}$. For each $j \in J, V \cap X_{j} \in \mathcal{T}_{j}$. Fix $j \in J$; for any point $p \in V \cap X_{j}$ there is a function $f \in \mathcal{F}_{j}$ such that $f^{-1}(0, \infty) \subset V \cap X_{j}$. Define $q: X \rightarrow \mathbb{R}$ by

$$
q(x)= \begin{cases}f(x) & \text { for } x \in X_{j} \\ 0 & \text { for } x \notin X_{j}\end{cases}
$$

Clearly,

$$
\left.q\right|_{X_{k}}= \begin{cases}f \in \mathcal{F}_{j} & \text { if } k=j \\ 0 \in \mathcal{F}_{k} & \text { if } k \neq j .\end{cases}
$$

Therefore $q \in \mathcal{F}$, and $q^{-1}(0, \infty)=f^{-1}(0, \infty)$. Since $j$ is arbitrary, $V \in \mathcal{T}$; and hence $\mathcal{T}^{\prime} \subset \mathcal{T}$.

Lemma 1.1.12 A differential space $(X, \mathcal{F})$ is Hausdorff if it satisfies any of the following equivalent conditions:

(1) For all $x, y \in X$, there exits a function $f \in \mathcal{F}$ such that $f(x) \neq f(y)$ if $x \neq y$.

(2) The $\mathcal{F}$-induced topology is Hausdorff.

Proof. (1) $\Rightarrow(2)$. Assume (1) and choose nonintersecting neighbourhoods $U$ and $V$ of $f(x)$ and $f(y)$ respectively. Subsets $f^{-1}(U)$ and $f^{-1}(V)$ are disjoint open neighbourhoods of $x$ and $y$ respectively. Thus, the $\mathcal{F}$-topology is Hausdorff. 
(2) $\Rightarrow(1)$. Suppose there exit $x \neq y$ in $X$ such that $f(x)=f(y)$ for all $f \in \mathcal{F}$. Since the $\mathcal{F}$-topology is Hausdorff, choose neighbourhoods $A$ and $B$, of $x$ and $y$ respectively, that are disjoint. There exist open neighbourhoods $T$ and $S$, of $f(x)$ and $f(y)$ respectively, such that $x \in f^{-1}(T) \subset A$ and $y \in f^{-1}(S) \subset B$. But, $f(x)=f(y)$, therefore $\{x, y\} \subset f^{-1}(T) \cap f^{-1}(S)$, and so $A \cap B \neq \emptyset$ : a contradiction to the assumption that $A \cap B=\emptyset$.

A differential space satisfying any of the above equivalent conditions is called a Hausdorff differential space.

We sould like to define quotient maps and quotient spaces in the category $\mathcal{D I F F}$ of differential spaces, which are necessary tools that we shall need later on in connection with adjunction spaces of differential spaces. We should also adapt Theorem 1.8 in [18] to the context of differential spaces. A corollary of Theorem 1.8 is used in [18] to show that the adjunction space of two topological spaces is unique up to a homeomorphism. A similar result holds in $\mathcal{D I F \mathcal { F }}$ for we shall prove that the adjunction of two differential spaces is unique up to a diffeomorphism.

Definition Let $X$ and $Y$ be differential spaces. A smooth surjection $q: X \rightarrow$ $Y$ is called a quotient map provided, given a real-valued function $f: Y \rightarrow \mathbb{R}$, then $f$ is smooth on $Y$ if and only if its pull-back $f \circ q$ is smooth on $X$.

Lemma 1.1.13 Let $X, Y, Z$ be differential spaces, and let $q: X \rightarrow Y$ be a quotient map. Then every set map $\Phi: Y \rightarrow Z$ such that $\Phi \circ q: X \rightarrow Z$ is 
smooth is a smooth map.

Proof. Let $\mathcal{F}(X), \mathcal{F}(Y)$ and $\mathcal{F}(Z)$ be collections of structure functions on $X, Y$ and $Z$ respectively. Let $h \in \mathcal{F}(Z)$. Then $h \circ \Phi \circ q \in \mathcal{F}(X)$. Since $q$ is a quotient, it follows that $h \circ \Phi \in \mathcal{F}(Y)$. Thus, $\Phi$ is smooth.

Definition Let $(X, \mathcal{F})$ be a differential space and let $X^{*}=\left\{X_{i}: i \in I\right\}$ be a partition of the set $X$. Let $\pi: X \rightarrow X^{*}$ be a set map defined by $\pi(x)=X_{i}$, where $X_{i}$ is the unique subset of $X$ in the partition $X^{*}$ containing $x$. The quotient differential structure $\pi^{*}(\mathcal{F})$ on $X^{*}$ is the coinduced structure corresponding to $\pi$, that is the collection of all real-valued functions $f: X^{*} \rightarrow$ $\mathbb{R}$ such that $f \circ \pi: X \rightarrow \mathbb{R}$ is smooth on $X$. The pair $\left(X^{*}, \pi^{*}(\mathcal{F})\right)$ is called the quotient space of $(X, \mathcal{F})$ determined by the natural map $\pi$.

Theorem 1.1.1 Let $X, Y$ be differential spaces and let $q: X \rightarrow Y$ be a smooth surjective map. Then $q$ is a quotient map if and only if, for all differential spaces $Z$, and all functions $g: Y \rightarrow Z$, one has $g$ smooth if and only if $g \circ q$ is smooth.

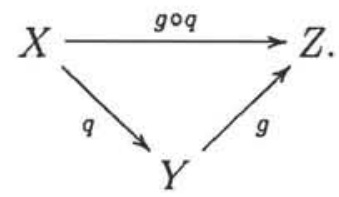

Proof. Assume $q$ is a quotient map. If $g$ is smooth, then $g \circ q$ is smooth. Conversely, let $g \circ q$ be smooth and let $h: Z \rightarrow \mathbb{R}$ be a smooth map on $Z$. Then $h \circ g \circ q: X \rightarrow \mathbb{R}$ is smooth; since $q$ is a quotient map, it follows that $h \circ g: Y \rightarrow \mathbb{R}$ is smooth. Hence $g$ is smooth. 
Now assume that it is true that for all differential spaces $Z$, and all functions $g: Y \rightarrow Z$, one has $g$ smooth if and only if $g \circ q$ is smooth. We want to show that this condition implies that $q$ is a quotient map. Let Ker $q$ be the equivalence relation on $X$, defined by $x \sim x^{\prime}$ if $q(x)=q\left(x^{\prime}\right)$. See [18]. Let $X / \operatorname{Ker}_{q}$ denote the quotient set of $X$ by $\operatorname{Ker} q$. It is clear that $X / \mathrm{Ker}_{q}$ is a partition of $X$. Let us use $X / \mathrm{Ker}_{q}$ for the corresponding quotient differential space. We apologize for abuse of notation. Let $Z=X / \operatorname{Ker}_{q}$ and let $\nu: X \rightarrow X / \operatorname{Ker}_{q}$ be the natural map. First note that there is an injection $\varphi: X /_{\mathrm{Ker}_{q}} \rightarrow Y$ defined by $\varphi([x])=q(x)$. But since $q$ is surjective, it follows that $\varphi$ is also surjective. Consider the commutative diagram

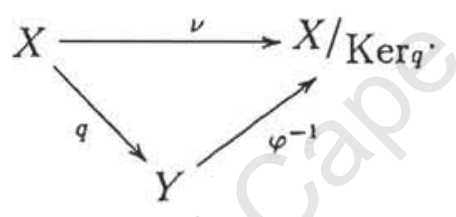

Since $\varphi^{-1} \circ q=\nu$ is smooth, it follows, by hypothesis, that $\varphi^{-1}$ is smooth. Moreover, since $\nu$ is a quotient and $\varphi \circ \nu=q$ is a smooth function, it follows that $\varphi$ is smooth. Thus, $\varphi$ is a diffeomorphism, and hence $q$ is a quotient map.

Corollary 1.1.2 Let $q: X \rightarrow Y$ be a quotient map of differential spaces and, for some differential space $Z$, let $h: X \rightarrow Z$ be a smooth map that is constant on every $q^{-1}(y)$, where $y \in Y$. Then $h \circ q^{-1}: Y \rightarrow Z$ is smooth.

Proof. That $h$ is constant on each $q^{-1}(y)$, where $y \in Y$, implies that $h \circ q^{-1}: Y \rightarrow Z$ is a well defined function; $h \circ q^{-1}$ is smooth because $\left(h \circ q^{-1}\right) \circ q=h$ is smooth, and Theorem 1.1.1 applies. 
Corollary 1.1.3 Let $X$ and $Z$ be differential spaces, and let $h: X \rightarrow Z$ be a quotient map. Then the map $\varphi: X /$ Kerh $\rightarrow Z$, defined by $[x] \mapsto h(x)$, is a diffeomorphism.

Proof. That $\varphi: X / \operatorname{Ker} h \rightarrow Z$ is a bijection is clear. Clearly, $\varphi$ is smooth by Corollary 1.1.2. Let $\nu: X \rightarrow X / \operatorname{Ker}_{h}$ be the natural map $x \mapsto[x]$. Since $h$ is a quotient map and $\nu$ is smooth, Theorem 1.1.1 implies that $\varphi^{-1}$ is smooth. 


\subsection{DW complexes}

DW complexes are analogues of CW complexes in the category of differential spaces. More precisely, a DW complex is a differential space made up of "cells", each of which is diffeomorphic to the interior of an euclidean unit ball; so each cell is viewed as a copy of an open ball, and it is said to have the dimension of the open ball. Cells are not allowed to overlap, they are rather attached in a systematic way. However, there are some difficulties with the way which they are attached across boundaries, to which we must attend.

Underlying spaces of CW complexes are assumed to be Hausdorff, likewise underlying spaces of DW complexes are required to be Hausdorff.

Throughout this section, $D^{k}$ will denote the unit $k$-ball, equipped with the differential structure $\mathcal{F}\left(D^{k}\right):=\mathcal{F}\left(\mathbb{R}^{k}\right)_{D^{k}}$ induced by the inclusion

$$
\iota_{D^{k}}: D^{k} \rightarrow\left(\mathbb{R}^{k}, \mathcal{F}\left(\mathbb{R}^{k}\right)\right)
$$

$\left(\mathcal{F}\left(\mathbb{R}^{k}\right)\right.$ is the usual differential structure of $\left.\mathbb{R}^{k}\right)$. Let us begin with

Lemma 1.2.1 Let $\left\{\left(X_{i}, \mathcal{F}_{i}\right) ; i \in I\right\}$ be a collection of differential spaces and, for each $i \in I$, let $f_{i}: X_{i} \rightarrow Y$ be a set function. Then

$$
f_{i}^{*}\left(\mathcal{F}_{i}\right)=\left\{g \in \mathbb{R}^{Y}: g \circ f_{i} \in \mathcal{F}_{i} \text { for each } i \in I\right\}
$$

is a differential structure on $Y$; it is called the coinduced structure relative to the family $\left\{f_{i}:\left(X_{i}, \mathcal{F}_{i}\right) \rightarrow Y\right\}_{i \in I}$. 
Proof. Let $h: Y \rightarrow \mathbb{R}$ be such that for every $p \in Y$ there is an open neighbourhood $U_{p}$ of $p$ in the topology underlying $f_{i}^{*}\left(\mathcal{F}_{i}\right)$ such that $\left.h\right|_{U_{p}}=$ $\left.g_{p}\right|_{U_{p}}$ for some $g_{p} \in f_{i}^{*}\left(\mathcal{F}_{i}\right)$. Since $f_{i}\left(f_{i}^{-1}\left(U_{p}\right)\right)=U_{p} \cap f_{i}\left(X_{i}\right)$, it follows that $\left.h \circ f_{i}\right|_{f_{i}^{-1}\left(U_{p}\right)}=\left.g_{p} \circ f_{i}\right|_{f_{i}^{-1}\left(U_{p}\right)}$. But $\left\{f_{i}^{-1}\left(U_{p}\right): p \in Y\right\}$ is an open covering of $X_{i}$ and $g_{p} \circ f_{i} \in \mathcal{F}_{i}$, therefore $h \circ f_{i} \in \mathcal{F}_{i}$. Thus $h \in f_{i}^{*}\left(\mathcal{F}_{i}\right)$. This property means that $f_{i}^{*}\left(\mathcal{F}_{i}\right)$ is closed with respect to localization.

Now we show that $f_{i}^{*}\left(\mathcal{F}_{i}\right)$ is closed with respect to composition with smooth functions $\omega \in C^{\infty}\left(\mathbb{R}^{n}, \mathbb{R}\right)$. Let $g_{1}, \ldots, g_{n} \in f_{i}^{*}\left(\mathcal{F}_{i}\right)$ and $\omega \in C^{\infty}\left(\mathbb{R}^{n}, \mathbb{R}\right)$. Clearly, one has $\nu \circ\left(g_{1}, \ldots, g_{n}\right) \circ f_{i}=\omega \circ\left(g_{1} \circ f_{i}, \ldots, g_{n} \circ f_{i}\right)$. Since $g_{j} \circ f_{i} \in \mathcal{F}_{i}$ for every $j=1, \ldots, n$, it follows that $\omega \circ\left(g_{1} \circ f_{i}, \ldots, g_{n} \circ f_{i}\right) \in \mathcal{F}_{i}$. Thus $\omega \circ\left(g_{1}, \ldots, g_{n}\right) \in f_{i}^{*}\left(\mathcal{F}_{i}\right)$.

For the time being let us assume that we know what a DW complex is.

Definition A $k$-cell $e^{k}$ (or simply $e$ ) of a DW complex $(X, \mathcal{F})$ is a subset of $X$, diffeomorphic to the interior of a closed ball $D^{k}$ via a smooth mapping $\Phi_{e}: D^{k} \rightarrow X$. The map $\Phi_{e}$ may identify points of $S^{k-1}$.

If $e^{\#}:=\Phi_{e}\left(D^{k}\right)$, then $e \subset e^{\#}$. We will denote by $\mathcal{F}\left(e^{\#}\right)$ the differential structure coinduced by $\Phi_{e}: D^{k} \rightarrow e^{\#}$ on $e^{\#}$, and by $\mathcal{T}\left(e^{\#}\right)$ the underlying topology of $\mathcal{F}\left(e^{\#}\right)$.

Definition Let $(X, \mathcal{F})$ be a DW complex with collection of cells $E$. For each $k \geq 0$, the $k$-dimensional skeleton $X^{(k)}$ of $X$ is the differential space whose underlying set is

$$
X^{(k)}=\cup\{e \in E: \operatorname{dim} e \leq k\},
$$


and whose differential structure is the structure coinduced by the inclusions $\left(e^{\#}, \mathcal{F}\left(e^{\#}\right)\right) \rightarrow X^{(k)}$.

Note the abuse of notation: $X^{(k)}$ is used to denote the $k$-dimensional skeleton as well as its underlying set. We will prove later that every skeleton is in fact a differential subspace of $X$.

Clearly, one gets in the category of sets the ascending sequence $X^{(0)} \subset X^{(1)} \subset$ $X^{(2)} \subset \ldots$ If there exists $n \in \mathbb{N}$ such that $X^{(n)}=X$ and $X^{(n-1)} \neq X^{(n)}$, then $X$ is said to be of dimension $n$. A DW complex is finite if it has finitely many cells. Using the above notions, we can now define:

Definition A DW complex or differential CW complex is a triple $((X, \mathcal{F}), E, \Phi)$, where $(X, \mathcal{F})$ is a Hausdorff differential space, $E$ is a family of cells, and $\Phi=\left\{\Phi_{e}: e \in E\right\}$ is a family of smooth maps, called characteristic maps, such that

(1d) $X=\cup\{e: e \in E\}$ (disjoint union).

(2d) For each $k$-zell $e \in E$, the smooth map $\Phi_{e}:\left(D^{k}, \mathcal{F}\left(D^{k}\right)\right) \rightarrow\left(e^{\#}, \mathcal{F}\left(e^{\#}\right)\right)$ is a quotient and carries the subspace $\left(\breve{D}^{k}, \mathcal{F}\left(D^{k}\right)_{D^{k}}\right)$ diffeomorphically onto $(e, \mathcal{F}(e))$, where $\mathcal{F}(e)$ is the structure induced by the inclusion $e \rightarrow\left(e^{\#}, \mathcal{F}\left(e^{\#}\right)\right)$.

(3d) If $e \in E$ is a $k$-cell, the subset $e^{\#} \subset X$ is contained in a finite union of some cells, in $E$, of dimension $\leq k$.

(4d) The differential structure $\mathcal{F}$ is the coinduced structure corresponding 
to the inclusions $\left(e^{\#}, \mathcal{F}\left(e^{\#}\right)\right) \rightarrow X$.

Conditions (2d) and (3d) imply that, given a $k$-cell $e$, the characteristic map $\Phi_{e}$ carries $S^{k-1}$ into the $k-1$ skeleton. More precisely, $S^{k-1}$ meets finitely many cells, each of which has dimension less than $k$. Therefore $e^{\#} \subset$ $e \cup X^{(k-1)}$. The underlying differential space $X$ is called a DW space, and $(E, \Phi)$ is called a DW decomposition of $X$. When there is fear of confusion about cells and chararcteristic maps of a DW complex $X$, we will adopt this rather more precise notation: $e_{\alpha}^{n}$ is the $\alpha$-th $n$-dimensional cell of $X$, and its corresponding characteristic map is denoted $\Phi_{\alpha}^{n}$. Also we may want to assign to each $n \geq 0$ a set $\Lambda_{n}$ indexing all the $n$-dimensional cells of $X$, so if $e_{\alpha}^{n}$ is an $n$-cell then $\alpha \in \Lambda_{n}$.

Lemma 1.2.2 Let $X$ be a $D W$ complex, let e be a $k$-dimensional cell in $X$ and let $h: D^{k} \rightarrow \mathbb{R}$ be a smooth map on $D^{k}$. If $\left.h\right|_{S^{k-1}}=\left.f \circ \Phi_{e}\right|_{S^{k-1}}$ for some smooth map $f: X^{(k-1)} \rightarrow \mathbb{R}$ then there is a unique smooth map $g: e^{\#} \rightarrow \mathbb{R}$ such that the diagram

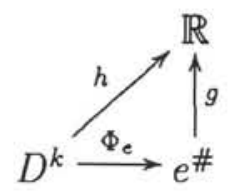

commutes and $\left.g\right|_{\partial e^{\#}}=\left.f\right|_{\partial e^{\#}}$, where $\partial e^{\#}=\Phi_{e}\left(S^{k-1}\right)$.

Proof. The reason is simply because the characteristic map $\Phi_{e}$ is a quotient and is one-to-one on the interior of $D^{k}$. In fact, take $g: e^{\#} \rightarrow \mathbb{R}$ as follows

$$
g(x)= \begin{cases}f(x) & \text { for } x \in \partial e^{\#} \\ \left.h\right|_{D^{k}} \circ\left(\left.\Phi_{e}\right|_{D^{k}}\right)^{-1}(x) & \text { for } x \in e .\end{cases}
$$


As $\partial e^{\#} \cap e=\emptyset, g$ is well defined and it is clear that $\left(g \circ \Phi_{e}\right)(x)=h(x)$ for all $x \in D^{k}$. Thus, as $\Phi_{e}$ is a quotient, $g$ is smooth on $e^{\#}$.

Lemma 1.2.3 Let $X$ be a $D W$ complex, let $\mathrm{D}=\sqcup_{\alpha \in \Lambda_{n}} \sqcup_{n \geq 0} D_{\alpha}^{n}$, where $\Lambda_{n}$ is the set indexing all the $n$-cells of $X$, be the coproduct of differential spaces $D_{\alpha}^{n}$, and let $q: \mathrm{D} \rightarrow X$ be the map $q=\underset{\substack{a \in \Lambda_{n} \\ n \geq 0}}{ } \Phi_{\alpha}^{n}$. ThenX has the structure coinduced by the map $q$.

Proof. By axiom (4d), if $\mathcal{F}$ is the differential structure of $X$, then

$$
\mathcal{F}=\left\{f \in \mathbb{R}^{X}:\left.f\right|_{e^{\#}} \in \mathcal{F}\left(e^{\#}\right), \text { for every } e \in E\right\}
$$

Now, if $\mathcal{D}$ is the structure coinduced on $\mathrm{D}$ by the inclusions $D_{\alpha}^{n} \rightarrow \mathrm{D}$, one has

$$
\mathcal{D}=\left\{f \in \mathbb{R}^{\mathrm{D}}:\left.f\right|_{D_{\alpha}^{n}} \in \mathcal{F}\left(D_{\alpha}^{n}\right)\right\}
$$

On the other hand, suppose

$$
\mathcal{G}=\left\{f \in \mathbb{R}^{X}: f \circ q \in \mathcal{D}\right\}
$$

Of course, $\mathcal{G}$ is the differential structure on $X$ coinduced by the map $q$. As asserted above, $f \in \mathcal{G}$ if and only if $f \circ q \in \mathcal{D}$, which in turn istrue if and only if $\left.f \circ q\right|_{D_{\alpha}^{n}}=\left.f\right|_{e^{\#}} \circ \Phi_{\alpha}^{n} \in \mathcal{F}\left(D_{\alpha}^{n}\right)$ for each $e_{\alpha}^{n}$. But $\left.f\right|_{e^{\#}} \circ \Phi_{\alpha}^{n} \in \mathcal{F}\left(D_{\alpha}^{n}\right)$ if and only if $\left.f\right|_{e^{\#}} \in \mathcal{F}\left(e^{\#}\right)$, which is equivalent to $f$ being in $\mathcal{F}$. (Note that we have omitted the subscript and superscript on $e^{\#}$. This usual attitude is adopted to circumvent unnecessary cumbersome details. Thus $\mathcal{G}=\mathcal{F}$, and hence $\mathcal{F}$ is the structure coinduced by the map $q$. 
Lemma 1.2.4 Let $(X, \mathcal{F}(X))$ be a $D W$ complex with cells $E(X)$, and let $(Y, \mathcal{F}(Y))$ be any differential space. A function $f: X \rightarrow Y$ is smooth if and only if $\left.f\right|_{e \#} \circ \Phi_{e}$ is smooth for each $e \in E(X)$.

Proof. Suppose that $f: X \rightarrow Y$ is smooth and let $e$ be a cell in $X$. It is clear that $\left.f\right|_{e^{\#}}: e^{\#} \rightarrow f\left(e^{\#}\right)$ is smooth. It follows that the composition $\left.f\right|_{e^{\#}} \circ \Phi_{e}: D^{k} \rightarrow f\left(e^{\#}\right)$ is also smooth.

Suppose that $f: X \rightarrow Y$ is such that $\left.f\right|_{e \#} \circ \Phi_{e}$ is smooth for each cell $e \in$ $E(X)$ and $g: Y \rightarrow \mathbb{R}$ is smooth on $Y$. Then every $\left.\left.g\right|_{f\left(e^{\#}\right)} \circ f\right|_{e^{\#}} \circ \Phi_{e}: D^{k} \rightarrow \mathbb{R}$ is smooth. Fix $e \in E(X)$. Since $\Phi_{e}: D^{k} \rightarrow e^{\#}$ is a quotient, it follows that $\left.\left.g\right|_{f(e \#)} \circ f\right|_{e^{\#}}$ is smooth on $e^{\#}$. But $e$ is arbitrary, therefore $g \circ f \in \mathcal{F}(X)$; hence $f$ is smooth.

Lemma 1.2.5 Let $\left(A, \mathcal{F}_{A}\right)$ be a differential subspace of a differential space $(X, \mathcal{F})$, and let $T_{A}$ and $\mathcal{T}$ be the topologies induced by $\mathcal{F}_{A}$ and $\mathcal{F}$ on $A$ and $X$ respectively. Then $\left(A, \mathcal{T}_{A}\right)$ is a topological subspace of $(X, \mathcal{T})$.

Proof. Let $\mathcal{S}$ be the topology on $A$, inherited from the topological space $(X, \mathcal{T})$. We claim that $\mathcal{S}=\mathcal{T}_{A}$. To show this, let us first note that

$$
\begin{gathered}
\mathcal{T}=\operatorname{gen}\left\{f^{-1}(W): W \in \mathcal{T}_{\mathbb{R}} \text { and } f \in \mathcal{F}\right\} \\
\mathcal{T}_{A}=\operatorname{gen}\left\{f^{-1}(W): W \in \mathcal{T}_{\mathbb{R}} \text { and } f \in \mathcal{F}_{A}\right\} \\
S=\operatorname{gen}\left\{f^{-1}(W) \cap A: W \in \mathcal{T}_{\mathbb{R}} \text { and } f \in \mathcal{F}\right\},
\end{gathered}
$$


where $\mathcal{T}_{\mathbb{R}}$ is the usual topology of $\mathbb{R}$. Since $f \in \mathcal{F}$ implies $\left.f\right|_{A} \in \mathcal{F}_{A}$, it follows that $\mathcal{S} \subset \mathcal{T}_{A}$.

For the reverse inclusion, observe that $f \in \mathcal{F}_{A}$ if and only if for each point $p \in A$, there is a neighbourhood $U_{p}$ in $\mathcal{T}$ such that $\left.f\right|_{U_{p} \cap A}=\left.f_{p}\right|_{U_{p} \cap A}$ for some function $f_{p} \in \mathcal{F}$. Since, for each $W \in \mathcal{T}_{\mathbb{R}}$,

$$
f^{-1}(W)=\cup_{p \in A}\left[f_{p}^{-1}(W) \cap U_{p} \cap A\right],
$$

it follows that $f^{-1}(W) \in \mathcal{S}$, and so $\mathcal{T}_{A} \subset \mathcal{S}$.

Lemma 1.2.6 Let $(X, \mathcal{F})$ be a differential space and let $B \subset A \subset X$. Then

$$
\mathcal{F}_{B}=\left(\mathcal{F}_{A}\right)_{B}
$$

Proof. It is easy to see that

$$
\begin{aligned}
\mathcal{F}_{B} & =\operatorname{gen}\left(\left\{\left.f\right|_{B} ; f \in \mathcal{F}\right\}\right) \\
& =\operatorname{gen}\left(\left\{\left.f\right|_{B} ;\left.f \in \mathcal{F}\right|_{A}\right\}\right) \\
& =\operatorname{gen}\left(\left\{\left.f\right|_{B} ; f \in \mathcal{F}_{A}\right\}\right), \text { since } \mathcal{F}_{A}=\operatorname{gen}\left(\left.\mathcal{F}\right|_{A}\right), \\
& =\left(\mathcal{F}_{A}\right)_{B} .
\end{aligned}
$$

Lemma 1.2.7 Let $X$ be a $D W$ complex and $e^{\#}=\Phi_{e}\left(D^{k}\right)$ with $\Phi_{e}$ being the characteristic map of a $k$-cell $e$ of $X$. Then if $\mathcal{F}\left(e^{\#}\right)$ is the differential 
structure coinduced by $\Phi_{e}$ on $e^{\#}$ and $\mathcal{F}_{e^{\#}}$ is the differential structure making the subset $e^{\#}$ into a differential subspace of $X$, it follows that

(i) $\mathcal{F}_{e^{\#}} \subset \mathcal{F}\left(e^{\#}\right)$

(ii) $\mathcal{T}_{\text {e\# }} \subset \mathcal{T}\left(e^{\#}\right)$,

where $\mathcal{T}_{e \#}$ and $\mathcal{T}\left(e^{\#}\right)$ are the underlying topologies of the structures $\mathcal{F}_{e^{\#}}$ and $\mathcal{F}\left(e^{\#}\right)$ repectively.

Proof. One easily obtains $(i)$ simply because $\mathcal{F}_{e^{\#}}=$ gen $\left\{\left.f\right|_{e^{\#}}: f \in \mathcal{F}\right\}$ and $\left.f\right|_{e^{\#}} \in \mathcal{F}\left(e^{\#}\right)$, for every $f \in \mathcal{F}$. Now, let $U$ be a subset of $e^{\#}$. Then $U \in \mathcal{T}_{e^{\#}}$ if and only if for each $p \in U$ there is a $f_{p} \in \mathcal{F}_{\text {e\# }}$ such that $p \in f_{p}^{-1}(0, \infty) \subset U$. But $\mathcal{F}_{e^{\#}} \subset \mathcal{F}\left(e^{\#}\right)$, therefore $U \in \mathcal{T}\left(e^{\#}\right)$, and hence $\mathcal{T}_{e^{\#}} \subset \mathcal{T}\left(e^{\#}\right)$.

Theorem 1.2.1 Let $(X, E)$ be a $D W$ complex with differential structure $\mathcal{F}$. Let $Y$ be a differential subspace of $X$ made up of cells of $X$, that is

$$
Y=\cup\left\{e \in E^{\prime} ; E^{\prime} \subset E\right\}
$$

Denote by $\Phi_{e}^{X}$ (resp. $\left.\Phi_{e}^{Y}\right)$ the characteristic map of a cell e of $X$ (resp. $Y$ ). Suppose that for each cell e of $X$ contained in $Y, \Phi_{e}^{Y}=\Phi_{e}^{X}$ and $e^{\#}$ is also contained in $Y$. Then $Y$ is a DW complex on its own right.

Proof. Let $x, y$ be any two points in $Y$. Since $X$ is Hausdorff it follows from Lemma 1.1.12 that there exits a smooth map $f: X \rightarrow \mathbb{R}$ on $X$ such that 
$f(x) \neq f(y)$. But $\left.f\right|_{Y}$ is smooth, so there is a smooth map $g=\left.f\right|_{Y}$ on $Y$ such that $g(x) \neq g(y)$. Thus, $Y$ is Hausdorff. If $e$ is a $k$-cell of $X$ contained in $Y$, then its characteristic map $\Phi_{e}: D^{k} \rightarrow X$ carries $D^{k}$ onto $e^{\#}$, which is contained in $Y$ by hypothesis. By (3d), there are finitely many cells $e_{i}$ of dimension leqk that meet $e^{\#}$. As $e^{\#} \subset Y$, it follows that every $e_{i}$ is also contained in $Y$. Thus, $e^{\#}$ is contained in some finite union of cells of $Y$.

It only remains to show that $Y$ has the differential structure specified by (4d).

Let $\mathcal{F}_{Y}$ be the differential structure on $Y$, induced by the inclusion $Y \rightarrow X$. Let $f \in \mathcal{F}_{Y}$. Then for each $p \in Y$, there is a neighbourhood $U_{p} \in \mathcal{T}$ such that $\left.f\right|_{U_{p} \cap Y}=\left.g\right|_{U_{p} \cap Y}$ for some $g \in \mathcal{F}$. If $p$ is a non 0-cell point contained in a cell $e$, then, by Lemma 1.2.7, $U_{p} \cap e^{\#}$ is an open set for the topology $\mathcal{T}\left(e^{\#}\right)$; and since $\left.f\right|_{U_{p} \cap e^{\#}}=\left.g\right|_{U_{p} \cap e^{\#}}$ with $\left.g\right|_{e^{\#}} \in \mathcal{F}\left(e^{\#}\right)$, it follows that $\left.f\right|_{e^{\#}} \in \mathcal{F}\left(e^{\#}\right)$. Suppose that $q$ is a 0 -cell in $Y$. It is obvious that $f(q) \in \mathcal{F}(q)$. Hence, if

$$
\mathcal{G}=\left\{f: Y \rightarrow \mathbb{R} ;\left.f\right|_{e^{\#}} \in \mathcal{F}\left(e^{\#}\right) \text { for all } e \in E^{\prime}\right\}
$$

then $\mathcal{F}_{Y} \subset \mathcal{G}$.

On the other hand, let us show that every map $f \in \mathcal{G}$ can be step by step extended to some smooth map $\hat{f}$, defined on the whole of the space $X$. Then, $f=\left.\hat{f}\right|_{Y} \in \mathcal{F}_{Y}$. The arguments given for the case whereby the complement $X-Y$ is made up of cells of dimension 2 at most can be applied to a more general case. Let $e_{\alpha}^{0}, \alpha \in I$, be all the 0 -cells contained in $X-Y$. Define $g: Y \cup\left(\cup_{\alpha \in I} e_{\alpha}^{0}\right) \rightarrow \mathbb{R}$ such that $\left.g\right|_{Y}=f$ and $g\left(e_{\alpha}^{0}\right)=1$ for all $\alpha \in I$. Suppose 
that, for each $\alpha \in J \subseteq I$, there is a 1-cell $e_{\alpha \beta}^{1}$

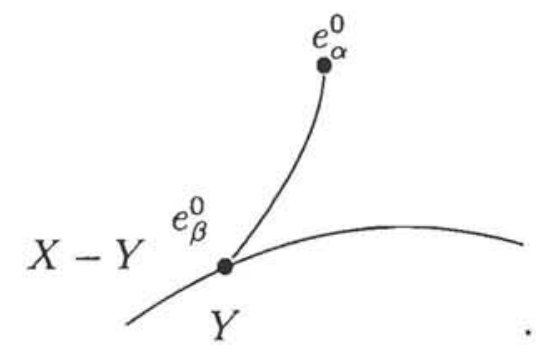

such that its one boundary point is the 0 -cell $e_{\alpha}^{0}$ in $X-Y$, while its other boundary point is some 0 -cell $e_{\beta}^{0}$ in $Y$. Denote by $\Phi_{\alpha \beta}^{1}$ the characteristic map of the cell $e_{\alpha \beta}^{1} ; \Phi_{\alpha \beta}^{1}$ sends $D^{1}$ into $X$. Assume that $\Phi_{\alpha \beta}^{1}(-1)=e_{\alpha}^{0}$ and $\Phi_{\alpha \beta}^{1}(1)=e_{\beta}^{0}$. For each such $e_{\alpha \beta}^{1}$, define $h_{\alpha \beta}^{1}: D^{1}-\{0\} \rightarrow \mathbb{R}$ by setting

$$
h_{\alpha \beta}^{1}(x)=\left\{\begin{array}{ll}
g\left(e_{\alpha}^{0}\right)=1 & \text { if }-1 \leq x<0 \\
g\left(e_{\beta}^{0}\right) & \text { if } 0<x \leq 1
\end{array},\right.
$$

and define $k_{\alpha \beta}^{1}: D^{1} \rightarrow \mathbb{R}$ by

$$
k_{\alpha \beta}^{1}(x)=\left\{\begin{array}{ll}
\lambda\left(|x|^{2}\right) h_{\alpha \beta}^{1}(x) & \text { if } x \neq 0 \\
0 & \text { if } x=0
\end{array},\right.
$$

where $\lambda: \mathbb{R} \rightarrow \mathbb{R}$ is a smooth function such that

$$
\lambda(x)= \begin{cases}1 & \text { if }|x| \geq \frac{3}{4} \\ 0 & \text { if }|x| \leq \frac{1}{2}\end{cases}
$$

Clearly, each $k_{\alpha \beta}^{1}$ is smooth. Since $\left.\Phi_{\alpha \beta}^{1}\right|_{(-1,1)}$ is a diffeomorphism onto $e_{\alpha \beta}^{1}$, there exists a map $g_{\alpha \beta}^{1}:\left(e_{\alpha \beta}^{1}\right)^{\#} \rightarrow \mathbb{R}$ such that $k_{\alpha \beta}^{1}=g_{\alpha \beta}^{1} \circ \Phi_{\alpha \beta}^{1}$.

The case where $e_{\alpha \beta}^{1}$ is a 1-cell in $X-Y$

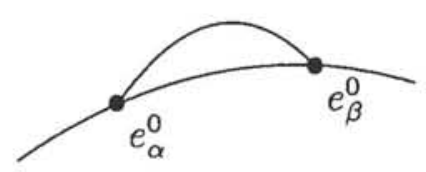


with its boundary points being distinct 0 -cells in $Y$ is treated similarly.

If $e_{\alpha \beta}^{1}$ is a cell in $X-Y$ whose boundary points $e_{\alpha}^{0}$ and $e_{\beta}^{0}$ lie in $X-Y$, define $g_{\alpha \beta}^{1}:\left(e_{\alpha \beta}^{1}\right)^{\#} \rightarrow \mathbb{R}$ to be identically 1.

Now, suppose that there also exist 1-cells $e_{\alpha \beta}^{1}$ in $X-Y$ such that $\Phi_{\alpha \beta}^{1}(-1)=$ $\Phi_{\alpha \beta}^{1}(1)$ is a 0 -cell in $Y$. One has the following picture

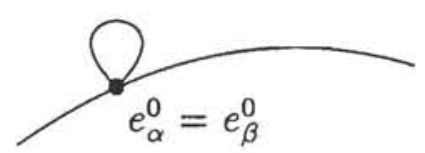

For such $e_{\alpha \beta}^{1}$, set $g_{\alpha \beta}^{1}(x)=f\left(e_{\alpha}^{0}\right)$ for all $x \in e_{\alpha \beta}^{1}$.

The various $g_{\alpha \beta}^{1}$ and the map $f$ patch together to define a map

$$
h: Y \cup(\cup\{e \in X-Y: \operatorname{dim} e \leq 1\}) \rightarrow \mathbb{R}
$$

such that $\left.h\right|_{Y}=f$ and for every 1-dimensional cell $e \in X-Y$ the restriction $\left.h\right|_{\text {e\# }}$ is smooth.

Now assume $e_{\alpha \beta \gamma}^{2}$ is a 2 -cell whose vertices $e_{\alpha}^{0}, e_{\beta}^{0}, e_{\gamma}^{0}$ are 0 -cells in $X-Y$, for instance in the diagram

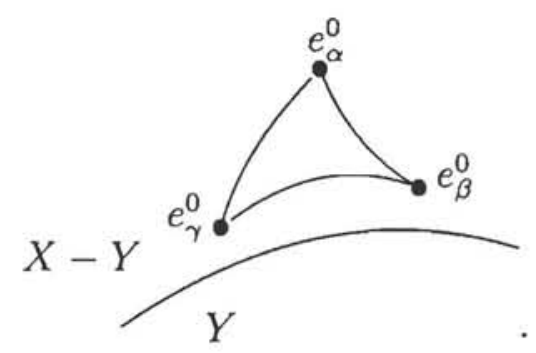

For such a cell, define $g_{\alpha \beta \gamma}^{2}:\left(e_{\alpha \beta \gamma}^{2}\right)^{\#} \rightarrow \mathbb{R}$ to be the constant map 1 . 
In turn, let $e_{\alpha \beta \gamma}^{2}$ be a 2-cell in $X-Y$ such that all its vertices are identified to a one 0 -cell of $Y$, that is $e_{\alpha}^{0}=e_{\beta}^{0}=e_{\gamma}^{0} \in Y$. It might be pictured as follows

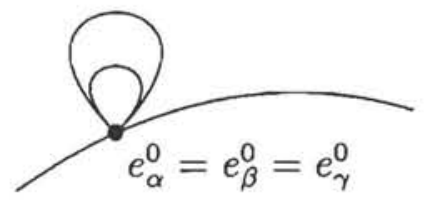

Define $g_{\alpha \beta \gamma}^{2}:\left(e_{\alpha \beta \gamma}^{2}\right)^{\#} \rightarrow \mathbb{R}$ to be the constant map $f\left(e_{\alpha}^{0}\right)$.

Next suppose $e_{\alpha \beta \gamma}^{2}$ is a 2-cell in $X-Y$

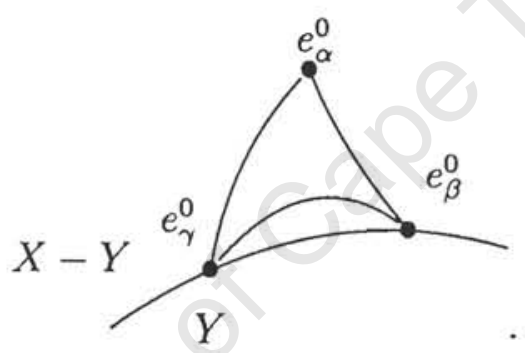

where $e_{\beta}^{0}$ and $e_{\alpha}^{0}$ are distinct 0 -cells in $Y$ and $e_{\alpha}^{0}$ is a 0 -cell in $X-Y$. If $\Phi_{\alpha \beta \gamma}^{2}$ is the characteristic map of the cell $e_{\alpha \beta \gamma}^{2}$, the function

$$
h_{\alpha \beta \gamma}^{1}=\left.\left(h \circ \Phi_{\alpha \beta \gamma}^{2}\right)\right|_{S^{1}}
$$

is a smooth map. Let $j^{2}: D^{2}-\{0\} \rightarrow \mathbb{R}$ be the map defined by

$$
j^{2}(x)=h_{\alpha \beta \gamma}^{1}(x /\|x\|)
$$

and let $h_{\alpha \beta \gamma}^{2}: D^{2} \rightarrow \mathbb{R}$ be given by

$$
h_{\alpha \beta \gamma}^{2}(x)= \begin{cases}\lambda\left(\|x\|^{2}\right) j^{2}(x) & \text { if } x \neq 0 \\ 0 & \text { if } x=0,\end{cases}
$$


where $\lambda: \mathbb{R} \rightarrow \mathbb{R}$ is defined as in (1.1). Clearly $h_{\alpha \beta \gamma}^{2}$ is a smooth map on $D^{2}$. Since

$$
\left.h_{\alpha \beta \gamma}^{2}\right|_{S^{1}}=\left.\left(h \circ \Phi_{\alpha \beta \gamma}^{2}\right)\right|_{S^{1}}
$$

and $\left.\Phi_{\alpha \beta \gamma}^{2}\right|_{\text {int } D^{2}}$ is a diffeomorphism onto $e$, it follows from Lemma 1.2.2 that there exists a smooth map $g_{\alpha \beta \gamma}^{2}:\left(e_{\alpha \beta \gamma}^{2}\right)^{\#} \rightarrow \mathbb{R}$ such that

$$
\left.g_{\alpha \beta \gamma}^{2}\right|_{\Phi_{\alpha \beta \gamma}^{2}\left(S^{1}\right)}=\left.h\right|_{\Phi_{\alpha \beta \gamma}^{2}\left(S^{1}\right)}
$$

and

$$
h_{\alpha \beta \gamma}^{2}=g_{\alpha \beta \gamma}^{2} \circ \Phi_{\alpha \beta \gamma}^{2} .
$$

The following cases
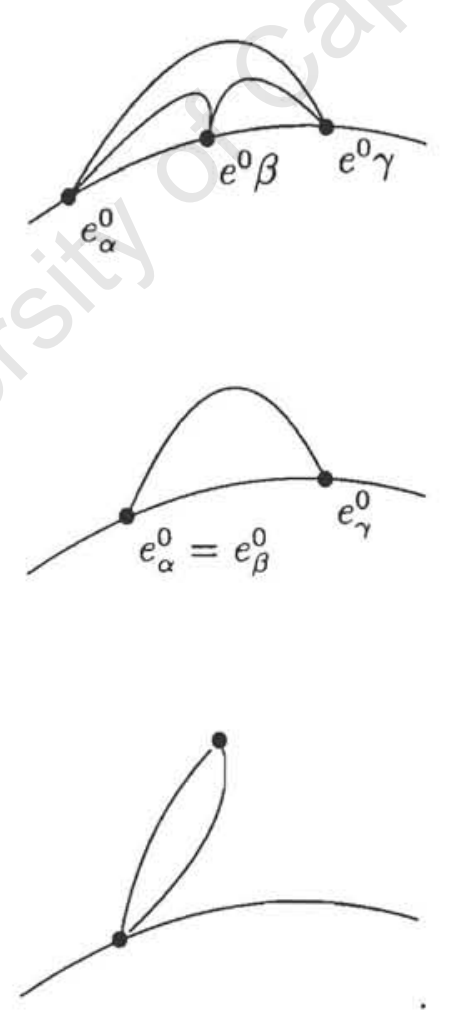
can be treated like the case just above.

Thus, by patching together the various $g_{\alpha \beta \gamma}^{2}$ and the map $h$, one extends $f$ to a smooth map $\hat{f}: X \rightarrow \mathbb{R}$. Hence $\left.\mathcal{G} \subset \mathcal{F}\right|_{Y}$, and consequently $\mathcal{G} \subset \mathcal{F}_{Y}$ as desired.

Definition A DW subcomplex $(Y, E(Y))$ of a DW complex $(X, E)$ is a DW complex such that $Y \subset X, E(Y) \subset E$ and $\Phi_{e}^{Y}=\Phi_{e}^{X}$ for every cell $e$ in $Y$.

In particular, $k$-skeletons of $X$ are DW subcomplexes of $X$.

Lemma 1.2.8 If $(X, E)$ is a $D W$ complex, with differential structure $\mathcal{F}$, and $e$ is any cell in $E$, then $e^{\#}$ is contained in a finite DW subcomplex, that is a DW subcomplex with a finite number of cells.

Proof. When $n=0$, the statement is obviously true. Now, suppose $n>0$; then by axiom (3d), $e^{\#}$ is contained in a finite union of cells, say $e_{1}, \ldots, e_{m}$. Define

$$
Y=\cup\left\{e_{i}^{\#}: 1 \leq i \leq m\right\}
$$

Taking $\Phi_{e}^{Y}=\Phi_{e}^{X}$ for every cell $e$ in $Y$, Theorem 1.2.1 implies that the differential subspace $\left(Y, \mathcal{F}_{Y}\right)$ is a DW subcomplex. It is finite and contains $e^{\#}$.

Lemma 1.2.9 If $(X, E)$ is a $D W$ complex and if $(Y, E(Y))$ and $(Z, E(Z))$ are $D W$ subcomplexes, then $Y \cap Z$ and $Y \cup Z$ are $D W$ subcomplexes with cells $E(Y) \cap E(Z)$ and $E(Y) \cup E(Z)$ respectively. 
Proof. It is clear that $Y \cap Z=\cup\{e: e \in E(Y) \cap E(Z)\}$ and for each cell $e$ in $E(Y) \cap E(Z)$, the subset $e^{\#}$ is in $Y \cap Z$. Since $e^{\#} \subset E(Y) \cap E(Z)$, it follows that $Y \cap Z=\cup\left\{e^{\#}: e \in E(Y) \cap E(Z)\right\}$; and by Theorem 1.2.1, $Y \cap Z$ is a DW complex and hence a DW subcomplex of $(X, E)$.

Now let us consider the subset $Y \cup Z$. Since $Y=\cup\left\{e^{\#}: e \in E(Y)\right\}$ and $Z=\cup\left\{e^{\#}: e \in E(Z)\right\}$, it follows that $Y \cup Z=\cup\left\{e^{\#}: e \in E(Y) \cup E(Z)\right\}$; so by Theorem 1.2.1 we corroborate the claim that $(Y \cup Z, E(Y) \cup E(Z))$ is a DW' subcomplex.

Corollary 1.2.1 If $(X, E)$ is a $D W$ complex and $K$ is a subset of $X$ finitely covered by cells of $X$, then $K$ lies in a finite $D W$ subcomplex.

Proof. Suppose $K^{\prime} \subset \cup_{i=1}^{m} e_{i}$, where every $e_{i}$ is a cell in $X$. By Lemma 1.2.8, $e_{i}^{\#}$ is contained in a finite DW subcomplex $Y_{e_{1}}$. So, $K \subset \cup_{i=1}^{m} Y_{e_{1}}$. But by Lemma 1.2.9. the union $\bigcup_{i=1}^{m} Y_{e_{t}}$ is a finite DW subcomplex. so the proof is finished.

Let $X$ be a DW complex; in addition to its underlying topology we place another topology, denoted $\sigma$, on $X$ by saying that a subset $C^{\prime}$ is open with respect to $\sigma$ if and only if, for each $k$-cell $e$ of $X$, the intersection $U^{\prime} \cap e^{\#}$ is open with respect to the smooth structure $\mathcal{F}\left(e^{\#}\right)$ of $e^{\#}$. (It follows that a set is closed for the topology $\sigma$ if and only if its intersection with each $e^{\#}$ is closed.) It is evident that $\sigma$ is truly a topology on $X$ and this is the topology that one might expect $X$ to have as a $\mathrm{CW}$ complex. 
Lemma 1.2.10 If $X$ is a DW complex and $e$ is a $k$-cell in $X$, then

$$
e^{\#}=c l_{\sigma}(e)
$$

where $\operatorname{cl}_{\sigma}(e)$ denotes the closure of $e$ with respect to the topology $\sigma$.

Proof. Since $e^{\#}$ is a closed set for the topology of $\sigma$, it follows that $\mathrm{cl}_{\sigma}(e) \subset$ $e^{\#}$. Now, assume that $\operatorname{cl}_{\sigma}(e)$ is strictly contained in $e^{\#}$. Then there exist a point $p$ in $e^{\#}$ and a neighbourhood $V$ of $p$ contained in $e^{\#}$ such that $V \cap e=\emptyset$. Since $p \in e^{\#}-\operatorname{cl}_{\sigma}(e)$, it follows that $p=\Phi_{e}(q)$ for some $q \in S^{k-1}$ ( we are assuming that $e$ is a cell of dimension $k$ ); moreover $q \in \Phi_{e}^{-1}(V)$ with $\Phi_{e}^{-1}(V)$ a neighbourhood of $q$. But the topology on $D^{k}$ is the topolgy inherited from the usual topology of $\mathbb{R}^{k}$, therefore $\Phi_{e}^{-1}(V)$ contains some points of $\breve{D}^{k}$. If $r$ is one such point, then $\Phi_{e}(r) \in V \cap e$; thus the assumption that $\mathrm{cl}_{\sigma}(e)$ is strictly contained in $e^{\#}$ does not hold. Hence, $\operatorname{cl}_{\sigma}(e)=e^{\#}$.

Similarly, one has

Lemma 1.2.11 Let $X$ be a $D W$ complex and let $\mathcal{T}$ be the initial topology corresponding to the differential structure $\mathcal{F}$. Then

$$
e^{\#}=\operatorname{cl}_{\mathcal{T}}(e),
$$

where $\operatorname{cl}_{\mathcal{T}}(e)$ is the closure of the cell e with respect to the topology $\mathcal{T}$.

Proof. First, note, for each $k$-cell $e, \Phi_{e}\left(\breve{D}^{k}\right)=e \subset \Phi_{e}\left(D^{k}\right)=e^{\#}$. As $D^{k}$ is compact and $X$ is Hausdorff, it follows that $\Phi_{e}\left(D^{k}\right)$ is a closed subset of $X$ and thus $\operatorname{cl}_{\mathcal{T}}(e) \subset e^{\#}$. 
Conversely, let $x \in e^{\#}$. Clearly there is a $y \in D^{k}$ such that $x=\Phi_{e}(y)$. If $y \in$ int $D^{k}$, then $x \in e$ and thus $x \in \operatorname{cl}_{\mathcal{T}}(e)$. Suppose $y \in S^{k-1}$. There exists a sequence of points $y_{i} \in \operatorname{int} D^{k}$ such that $y_{i} \rightarrow y$ as $i \rightarrow \infty$. Since $\Phi_{e}\left(y_{i}\right) \in e$ and $\Phi_{e}$ is continuous, it follows that $\Phi_{e}\left(y_{i}\right) \rightarrow \Phi_{e}(y)=x$; and one sees that $x \in \operatorname{cl}_{\mathcal{T}}(e)$, hence $e^{\#} \subset \operatorname{cl}_{\mathcal{T}}(e)$.

Putting Lemma 1.2.10 and Lemma 1.2.11 together, one obtains

Corollary 1.2.2 Let $(X, \mathcal{F})$ be a $D W$ complex, let $\mathcal{T}$ be the underlying topology of $\mathcal{F}$ and let $\sigma$ be a topology on $X$ whose open sets are those subsets $U$ of $X$ such that $U \cap e^{\#} \in \mathcal{T}\left(e^{\#}\right)$ for each cell e of $X$. Then,

$$
c l_{\mathcal{T}}(e)=e^{\#}=\operatorname{cl}_{\sigma}(e)
$$

for each cell e of $X$.

Because of the foregoing equations, we shall use the notation $\bar{e}$ to denote the closure of $e$ for eicher the topology $\sigma$ or the topology $\mathcal{T}$. If $e$ is a $k$-dimensional cell of the DW complex, $\bar{e}$ will be called a closed $k$-cell of $X$.

Lemma 1.2.12 If $(X, E, \Phi)$ is a $D W$ complex, then the triple $((X, \sigma), E, \Phi)$ is a CW complex.

Proof. Axioms (1R), (2R) in the definition of CW complexes are readily satisfied. By Corollary 1.2.2, $\bar{e}=e^{\#}$; and since $e^{\#}$ is contained in a finite union of cells of lower dimensions, then (3R) holds. By definition of the 
topology of $\sigma$, a subset $U$ of $X$ is closed if and only if $U \cap \bar{e}$ is closed in $\bar{e}$; hence (4R) holds. Thus $((X, \sigma), E, \Phi)$ is a $\mathrm{CW}$ complex.

The following lemma will be useful for the main theorem, that we are about to prove.

Lemma 1.2.13 Let $p$ be a 0 -cell of $X$ and an isolated point of $X^{(1)}$ ( for relative topology induced from $\sigma$ ). Then, $p$ is an isolated point of $X$.

Proof. Suppose that $p$ is not an isolated point of $X$. Then, there is a closed $k$-cell $\bar{e}(k>1)$ such that $p \in \partial e$. Suppose also that there is no closed $l$-cell $\bar{o}$, where $1 \leq l<k$, such that $p \in \bar{o}$. Since $(X, \sigma)$ is a CW complex, the set $\partial e$ is finitely covered by $l$-cells $o$ and 0 -cells. Let

$$
\mathcal{O}=\{o \in E: 1 \leq \operatorname{dim} o<k \text { and } o \subset \partial e\} .
$$

As $p \notin \bar{o}$ for each $o \in \mathcal{O}$, there is an open neighbourhood $U_{o}$ of $p$ containing no other 0 -cell and such that $U_{o} \cap \bar{o}=\emptyset$. It follows that $p \notin \partial e$, which is a contradiction; the proof is therefore finished.

We have stated that the most interesting question for our dissertation is to show whether underlying spaces of DW complexes are CW complexes. To this end it suffices to show that topologies $\mathcal{T}$ and $\sigma$ are the same. But at the moment, we basically show one direction; that is $\mathcal{T}$ is finer than $\sigma$. In this particular situation, our approach is to consider two cases. For the first case, we let $p$ be a point in $e^{k} \cap U$, where $e^{k}$ is a $k$-cell and $U$ is an open set in the topology $\sigma$. For the second case, we allow $p$ to be a 0 -cell point in $U$. 
In both cases, we prove that there is a smooth function $f: X \rightarrow \mathbb{R}$ such that $p \in f^{-1}(0, \infty) \subset U$.

However, the first case has some complexities, for if $p$ is an interior point of some cell $e^{k}$ and $e^{k}$ belongs to the boundary of some cell $e_{\alpha}^{k+1}$, then it takes some effort to show how one gets a function $f: X \rightarrow \mathbb{R}$ with the desired property that $p \in f^{-1}(0, \infty) \subset U$.

Theorem 1.2.2 Let $(X, \mathcal{F})$ be a $D W$ complex, let $U$ be an open set in the topology $\sigma$, and let $p \in U$ be a non 0-cell point of $X$. Then there exists a smooth map $f \in \mathcal{F}$ such that

$$
p \in f^{-1}(0, \infty) \subset U
$$

Proof. Since $p$ is a non 0 -cell point of $X$, there exists a closed $k$-cell $\overline{e^{k}(p)}=$ $\overline{e^{k}}(k \geq 1)$ that contains the point $p$.

A) We begin by examining the case where $p$ is a point of $e^{k}$. We will show that that there is a smooth map $f^{k}: X^{(k)} \rightarrow \mathbb{R}$ such that

$$
p \in\left(f^{k}\right)^{-1}(0, \infty) \subset U \cap X^{(k)},
$$

and $f^{k}$ can be extended to some smooth map $f^{k+1}: X^{(k+1)} \rightarrow \mathbb{R}$ such that

$$
p \in\left(f^{k+1}\right)^{-1}(0, \infty) \subset U \cap X^{(k+1)} .
$$


The map $f^{k+1}$, in turn, can be extended to some smooth $f^{k+2}: X^{(k+2)} \rightarrow \mathbb{R}$ such that

$$
p \in\left(f^{k+2}\right)^{-1}(0, \infty) \subset U \cap X^{(k+2)},
$$

and so on ... So ultimately we obtain a sequence $\left\{f^{k+i}\right\}_{i \geq 0}$ of smooth maps $f^{k+i}: X^{(k+i)} \rightarrow \mathbb{R}$ such that

$$
p \in\left(f^{k+i}\right)^{-1}(0, \infty) \subset L^{\prime} \cap X^{(k+i)}
$$

for each $i \geq 0$. Thus if we let $f: X \rightarrow \mathbb{R}$ be such that $\left.f\right|_{X^{(k+i)}}=f^{k+i}$ for all $i \geq 0$, then one has the required property that $p \in f^{-1}(0, \infty) \subset U$ for some smooth map $f$.

First let us show that when $p$ lies in $e^{k}$, there exists indeed a smooth map $f^{k}: X^{(k)} \rightarrow \mathbb{R}$ such that $p \in\left(f^{k}\right)^{-1}(0, \infty) \subset U \cap X^{(k)}$.

Since $p$ is a point of $e^{k}$, there exists $q$ in the interior of $D^{k}$ such that $p=$ $\Phi_{e^{k}}(q)$, where $\Phi_{e^{k}}$ is the characteristic map associated to the cell $e^{k}$. Let $h, k: D^{k} \rightarrow \mathbb{R}$ be: a smooth map defined by

$$
h_{e^{k}}(x)=\left\{\begin{array}{ll}
1 & \text { for } x=q \\
>0 & \text { for } x \in D^{k} \text { such that }\|x-q\|<\epsilon / 2 \\
0 & \text { for } x \in D^{k} \text { such that }\|x-q\|=\epsilon / 2 \\
<0 & \text { for } x \in D^{k} \text { such that }\|x-q\|>\epsilon / 2 \\
-1 & \text { for } x \in S^{k-1}
\end{array},\right.
$$

where $\epsilon$ is chosen in such a way that each point $x$ in $D^{k}$ such that if $x$ satisfies $\|x-q\| \leq \epsilon$, then $x$ is contained in $\Phi_{e^{k}}^{-1}(U)$. The existence of $\epsilon$ is guaranteed by the fact that $q \in \Phi_{e^{k}}^{-1}\left(L^{\prime}\right)$, with $\Phi_{e^{k}}^{-1}\left(L^{\prime}\right)$ an open subset of $D^{k}$. Since 
$\left.h_{e^{k}}\right|_{S^{k-1}}=\left.(-1) \circ \Phi_{e^{k}}\right|_{S^{k-1}}$, where $(-1)(x)=-1$ for all $x \in X^{(k-1)}$, it follows from Lemma 1.2.2 that $h_{e^{k}}$ induces a unique smooth map $f_{e^{k}}: \overline{e^{k}} \rightarrow \mathbb{R}$ such that the diagram

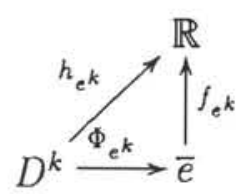

commutes. By construction, it follows that $h_{e^{k}}^{-1}(0, \infty)$ is contained in the interior of $\Phi_{e^{k}}^{-1}\left(U^{\prime}\right)$ and is such that

$$
\overline{h_{e^{k}}^{-1}(0, \infty)} \cap \operatorname{Fr} \Phi_{e^{k}}^{-1}(U)=\emptyset .
$$

Since $\Phi_{e^{k}}$ is a diffeomorphism from the interior of $D^{k}$ onto $e^{k}$, it follows that $f_{e^{k}}^{-1}(0, \infty)$ is contained in $U \cap e^{k}$ and is such that

$$
\overline{f_{e^{k}}^{-1}(0, \infty)} \cap \operatorname{Fr}\left(U \cap e^{k}\right)=\emptyset
$$

Then define $f^{k}: X^{(k)} \rightarrow \mathbb{R}$ to be the map

$$
f^{k}(x)=\left\{\begin{array}{cl}
f_{e^{k}}(x) & \text { for } x \in e^{k} \\
-1 & \text { otherwise }
\end{array} .\right.
$$

It is clear that $f^{k}$ is smooth, and $\left(f^{k}\right)^{-1}(0, \infty)=f_{e^{k}}^{-1}(0, \infty)$. So, one has

$$
p \in\left(f^{k}\right)^{-1}(0, \infty) \subset U \cap X^{(k)} \text {. }
$$

Next let us extend $f^{k}$ to the $(k+1)$-skeleton $X^{(k+1)}$. Two cases arise. 1) Suppose there is no cell $e^{j}(j>k)$ in $X$ such that its boundary $\partial e^{j}$ contains the cell $e^{k}$. If this is so, define a map $g: X^{(k+1)} \rightarrow \mathbb{R}$ by setting

$$
g(x)= \begin{cases}f_{e^{k}}(x) & \text { for } x \in e^{k} \\ -1 & \text { otherwise }\end{cases}
$$


It follows clearly that $g$ is smooth, and $p \in g^{-1}(0, \infty) \subset U \cap X^{(k+1)}$; so in this particular case one is finished.

2) Now suppose that $e^{k}$ belongs to the boundary of some $(k+1)$-cell $e^{*}$ of $X$. Then $q \in S^{k}$ and since $U \cap \overline{e^{k}} \subset U \cap \overline{e^{*}}$ with $U \cap \overline{e^{k}} \neq \emptyset$, it follows that $U \cap \overline{e^{*}} \neq \emptyset$. As $U$ is open in the topology $\sigma, U \cap \overline{e^{*}}$ is a non-empty open subset of $\overline{e^{*}}$. Thus $U \cap \overline{e^{*}}$ is of dimension $k+1$. Define $\hat{h}_{e^{*}}: S^{k} \rightarrow \mathbb{R}$ by setting

$$
\hat{h}_{e^{*}}=\left.\left(f^{k} \circ \Phi_{e^{*}}\right)\right|_{S^{k}}
$$

where $\Phi_{e^{*}}$ is the characteristic map associated to the cell $e^{*}$ and where $f^{k}$ is given by (1.2). Since $\Phi_{e^{*}}^{-1}(U)$ is open in $D^{k+1}$, there exists a set

$$
C_{\delta}^{k+1}=\left\{x \in D^{k+1} \cap \Phi_{e^{*}}^{-1}(U):\|x\|>1-\delta \text { and } \hat{h}_{e^{*}}(x /\|x\|)>0\right\}
$$

for some sufficiently small $\delta>0$ such that

$$
\operatorname{Fr} C_{\delta}^{k+1} \cap \operatorname{Fr} \Phi_{e^{*}}^{-1}(U) \cap \breve{D}^{k+1}=\emptyset .
$$

It can be easily seen that

$$
C_{\delta}^{k+1} \cap S^{k}=\hat{h}_{e^{*}}^{-1}(0, \infty)
$$

Moreover, since $\operatorname{Fr}\left(\Phi_{e^{*}}^{-1}(U) \cap S^{k}\right) \cap \overline{\hat{h}_{e^{*}}^{-1}(0, \infty)}=\emptyset$, it follows that $\overline{C_{\delta}^{k+1} \cap S^{k}}$ is contained in the interior of $\overline{\Phi_{e^{*}}^{-1}(U) \cap S^{k}}$. Therefore given a geodesic $L$ through $q$, if $r$ and $s$ are intersection points of $L$ with $\overline{C_{\delta}^{k+1} \cap S^{k}}$ and $\overline{\Phi_{e^{*}}^{-1}(U) \cap S^{k}}$ respectively on the same side of $q$, then

$$
\operatorname{dist}(q, s)>\operatorname{dist}(q, r)>0 \text {. }
$$


('dist' stands for distance.)

For the same $\delta$ above, let $u_{1}, u_{2}: \mathbb{R} \rightarrow \mathbb{R}$ be smooth maps defined by

$$
u_{1}(t)= \begin{cases}1 & \text { if }|t| \geq 1-\frac{1}{4} \delta \\ >0 & \text { if } 1-\frac{1}{4} \delta \geq|t|>1-\frac{3}{4} \delta \\ 0 & \text { if }|t| \leq 1-\frac{3}{4} \delta\end{cases}
$$

and

$$
u_{2}(t)= \begin{cases}0 & \text { if }|t| \geq 1-\frac{1}{4} \delta \\ >0 & \text { if } 1-\frac{1}{4} \delta>|t|>1-\frac{3}{4} \delta \\ 0 & \text { if }|t|=1-\frac{3}{4} \delta \\ <0 & \text { if }|t|<1-\frac{3}{4} \delta\end{cases}
$$

respectively. Let $h_{e^{*}}: D^{k+1} \rightarrow \mathbb{R}$ be a smooth map defined by

$$
h_{e^{*}}(x)=\left\{\begin{array}{ll}
u_{2}\left(\|x\|^{2}\right)+u_{1}\left(\|x\|^{2}\right) \hat{h}_{e^{*}}(x /\|x\|) & \text { for } x \neq 0 \\
u_{2}(0) & \text { for } x=0
\end{array} .\right.
$$

Since $h_{e^{*}}: D^{k+1} \rightarrow \mathbb{R}$ is smooth, $\left.h_{e^{*}}\right|_{S^{k}}=\left.\left(f^{k} \circ \Phi_{e^{*}}\right)\right|_{S^{k}}$, and $\left.\Phi_{e^{*}}\right|_{D^{k+1}}$ is a diffeomorphism onto $e^{*}$, Lemma 1.2.2 applies at once to show that there is a unique smooth map $f_{e^{*}}: \overline{e^{*}} \rightarrow \mathbb{R}$ making the diagram

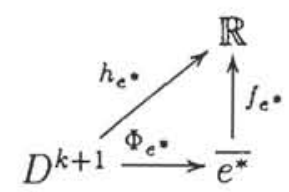

commute and such that

$$
\left.f_{e^{*}}\right|_{\partial e^{*}}=\left.f^{k}\right|_{\partial e^{*}}
$$


As $\hat{h}_{e^{*}}^{-1}(0, \infty)=C_{\delta}^{k+1} \cap S^{k}$ and $\left.h_{e^{*}}\right|_{S^{k}}=\hat{h}_{e^{*}}$, it follows that $h_{e^{*}}^{-1}(0, \infty) \cap S^{k}$ is contained in the interior of $\overline{\Phi_{e^{*}}^{-1}(\bar{U}) \cap S^{k}}$. Since by construction

$$
h_{e^{*}}^{-1}(0, \infty) \cap \breve{D}^{k+1} \subset \Phi_{e^{*}}^{-1}(U)
$$

one has that

$$
h_{e^{*}}^{-1}(0, \infty) \subset \Phi_{e^{*}}^{-1}(U)
$$

and

$$
\left.\operatorname{Fr}\left(h_{e^{*}}^{-1}(0, \infty)\right) \cap \operatorname{Fr}\left(\Phi_{e^{*}}^{-1}(U)\right)\right) \cap \breve{D}^{k+1}=\emptyset .
$$

Hence,

$$
f_{e^{*}}^{-1}(0, \infty) \subset U \cap \overline{e^{*}}
$$

and

$$
\operatorname{Fr}\left(f_{e^{*}}^{-1}(0, \infty)\right) \cap \operatorname{Fr}\left(U \cap \overline{e^{*}}\right) \cap e^{*}=\emptyset
$$

Let $\left\{\overline{e_{\alpha}^{k+1}}: \alpha \in \Lambda\right\}$ be the family of all closed $(k+1)$-cells whose boundaries contain the cell $e^{k}$. Of course, there is a $\alpha \in \Lambda$ such that $\overline{e^{*}}=\overline{e_{\alpha}^{k+1}}$. For each $\alpha \in \Lambda$, let

$$
\hat{h}_{e_{\alpha}^{k+1}}=\left.\left(f^{k} \circ \Phi_{e_{\alpha}^{k+1}}\right)\right|_{S^{k}}
$$

where $\Phi_{e_{\alpha}^{k+1}}$ is the characteristic map of the cell $e_{\alpha}^{k+1}$. Since $U \cap e_{\alpha}^{k+1} \neq \emptyset$ for each $\alpha \in \Lambda$, there exist a sufficiently small $\delta_{\alpha}>0$ and a set

$$
C_{\delta_{\alpha}}^{k+1}=\left\{x \in D^{k+1} \cap \Phi_{e_{\alpha}^{k+1}}^{-1}(U):\|x\|>1-\delta_{\alpha} \text { and } \hat{h}_{e_{\alpha}^{k+1}}(x /\|x\|)>0\right\}
$$


for each $\alpha \in \Lambda$ such that

$$
\operatorname{Fr}\left(C_{\delta_{\alpha}}^{k+1}\right) \cap \operatorname{Fr}\left(\Phi_{e_{\alpha}^{k+1}}^{-1}(U)\right) \cap \breve{D}^{k+1}=\emptyset .
$$

Here as well, one can easily see that $\overline{C_{\delta_{a}}^{k+1} \cap S^{k}}$ is contained in the interior of $\overline{\Phi_{e_{\alpha}^{k+1}}^{-1}\left(L^{r}\right) \cap S^{k}}$. If $r_{\alpha}$ and $s_{\alpha}$ are on boundaries of $\overline{C_{\delta_{\alpha}}^{k+1} \cap S^{k}}$ and $\frac{\Phi_{\alpha}^{k}}{\Phi_{e_{\alpha}^{k+1}}^{-1}(U) \cap S^{k}}$ respectively and lie on a same geodesic that passes through $q$, on a same side of $q$, then one has

$$
\operatorname{dist}\left(q, s_{\alpha}\right)>\operatorname{dist}\left(q, r_{\alpha}\right)>0 \text {. }
$$

Define for each $\alpha \in \Lambda$ a smooth map $h_{e_{\alpha}^{k+1}}: D^{k+1} \rightarrow \mathbb{R}$ by setting

$$
h_{e_{\alpha}^{k+1}}(x)= \begin{cases}u_{2, \alpha}\left(\|x\|^{2}\right)+u_{1, \alpha}\left(\|x\|^{2}\right) \hat{h}_{e_{\alpha}^{k+1}}(x /\|x\|) & \text { for } x \neq 0 \\ u_{2, \alpha}(0) & \text { for } x=0\end{cases}
$$

where

$$
u_{1, \alpha}(t)= \begin{cases}1 & \text { if }|t| \geq 1-\frac{1}{4} \delta_{\alpha} \\ >0 & \text { if } 1-\frac{1}{4} \delta_{\alpha} \geq|t|>1-\frac{3}{4} \delta_{\alpha} \\ 0 & \text { if }|t| \leq 1-\frac{3}{4} \delta_{\alpha}\end{cases}
$$

and

$$
u_{2, \alpha}(t)= \begin{cases}0 & \text { if }|t| \geq 1-\frac{1}{4} \delta_{\alpha} \\ >0 & \text { if } 1-\frac{1}{4} \delta_{\alpha}>|t|>1-\frac{3}{4} \delta_{\alpha} \\ 0 & \text { if }|t|=1-\frac{3}{4} \delta_{\alpha} \\ <0 & \text { if }|t|<1-\frac{3}{4} \delta_{\alpha} .\end{cases}
$$

For each $\alpha \in \Lambda$ we note that $\left.h_{e_{\alpha}^{k+1}}\right|_{S^{k}}=\left.\left(f^{k} \circ \Phi_{e_{\alpha}^{k+1}}\right)\right|_{S^{k}}$; so Lemma 1.2.2 implies that there is a unique smooth map $f_{e_{\alpha}^{k+1}}: \overline{e_{\alpha}^{k+1}} \rightarrow \mathbb{R}$ such that

$$
\begin{aligned}
h_{e_{\alpha}^{k+1}} & =f_{e_{\alpha}^{k+1}} \circ \Phi_{e_{\alpha}^{k+1}} \\
\left.f_{e_{\alpha}^{k+1}}\right|_{\partial e_{\alpha}^{k+1}} & =\left.f^{k}\right|_{\partial e_{\alpha}^{k+1}} .
\end{aligned}
$$


For each $\alpha \in \Lambda$,

$$
\left(\hat{h}_{e_{\alpha}^{k+1}}\right)^{-1}(0, \infty) \subset \Phi_{e_{\alpha}^{k+1}}^{-1}(U) .
$$

It follows that

$$
h_{e_{\alpha}^{k+1}}^{-1}(0, \infty) \subset \Phi_{e_{\alpha}^{k+1}}^{-1}(U)
$$

and

$$
\operatorname{Fr}\left(h_{e_{\alpha}^{k+1}}^{-1}(0, \infty)\right) \cap \operatorname{Fr}\left(\Phi_{e_{\alpha}^{k+1}}^{-1}(U)\right) \cap \breve{D}^{k+1}=\emptyset .
$$

Therefore,

$$
f_{e_{\alpha}^{k+1}}^{-1}(0, \infty) \subset U \cap \overline{e_{\alpha}^{k+1}}
$$

and

$$
\operatorname{Fr}\left(f_{e_{\alpha}^{k+1}}^{-1}(0, \infty)\right) \cap \operatorname{Fr}\left(U \cap \overline{e_{\alpha}^{k+1}}\right) \cap e_{\alpha}^{k+1}=\emptyset,
$$

for each $\alpha \in \Lambda$. Now consider the family $\left\{\overline{e_{\delta}^{k+1}}: \delta \in \Delta\right\}$ of closed cells of dimension $k+1$, which do not contain the cell $e^{k}$; thus $p \notin \overline{e_{\delta}^{k+1}}$ for each $\delta \in \Delta$. Set, for $\delta \in \Delta$,

$$
f_{e_{\delta}^{k+1}}: \overline{e_{\delta}^{k+1}} \rightarrow \mathbb{R}
$$

to be the constant map -1 . As

$$
\left.f_{e_{\alpha}^{k+1}}\right|_{X^{(k)} \cap e_{\alpha}^{k+1}}=\left.f^{k}\right|_{X^{(k)} \cap \overline{e_{\alpha}^{k+1}}}
$$

for each $\alpha \in \Lambda$, one can patch the $\left\{f_{e_{\alpha}^{k+1}}\right\}_{\alpha \in \Lambda},\left\{f_{e_{\delta}^{k+1}}\right\}_{\delta \in \Delta}$ and $f^{k}$ together to obtain a smooth map

$$
f^{k+1}: X^{(k+1)} \rightarrow \mathbb{R}
$$


such that

$$
p \in\left(f^{k+1}\right)^{-1}(0, \infty) \subset U \cap X^{(k+1)} .
$$

Since $\left.f^{k+1}\right|_{X^{(k)}}=f^{k}$, it follows that $f^{k+1}$ is a smooth extension of $f^{k}$.

Now let us extend $f^{k+1}$ smoothly to the skeleton $X^{(k+2)}$ so as to get a smooth map $f^{k+2}: X^{(k+2)} \rightarrow \mathbb{R}$ such that

$$
p \in\left(f^{k+2}\right)^{-1}(0, \infty) \subset U \cap X^{(k+2)} .
$$

As a particular case suppose there is no closed cell in $X$ containing $e^{*}$, and suppose as well that there is no closed cell in $X$ containing any of the cells $e_{\alpha}^{k+1}, \alpha \in \Lambda$. Thus there is no cell $e^{j}(j>k+1)$ in $X$ such that its boundary contains the point $p$. Let $h: X^{(k+2)} \rightarrow \mathbb{R}$ be the map defined by

$$
h(x)= \begin{cases}f^{k+1}(x) & \text { for } x \in X^{(k+1)} \\ -1 & \text { otherwise. }\end{cases}
$$

The map $h$ is easily seen to be smooth and such that $p \in h^{-1}(0, \infty) \subset U$.

Now suppose that $e^{*}$ is contained in the boundary of some closed $(k+2)$-cell $\overline{\tilde{e}}$ and suppose also that some of the cells $\overline{e_{\alpha}^{k+1}}, \alpha \in \Lambda$ are contained in closed cells of dimension $k+2$. Without loss of generality suppose, apart from $e^{*}$, that there is only one $\alpha \in \Lambda$ for which the corresponding $\overline{e_{\alpha}^{k+1}}$ is contained in the boundary of a closed $(k+2)$-cell $\overline{e_{\beta}^{k+2}}$. Define

$$
\hat{h}_{\tilde{e}}=\left.\left(f^{k+1} \circ \Phi_{\bar{e}}\right)\right|_{S^{k+1}}
$$

and

$$
\hat{h}_{e_{\beta}^{k+2}}=\left.\left(f^{k+1} \circ \Phi_{e_{\beta}^{k+2}}\right)\right|_{S^{k+1}} .
$$


Going through the same steps, which led us to extend $f^{k}$ to $f^{k+1}$, one proves that maps $h_{\bar{e}}, h_{e_{\beta}^{k+2}}: D^{k+2} \rightarrow \mathbb{R}$ defined respectively by

$$
h_{\bar{e}}(x)= \begin{cases}u_{2, \kappa}\left(\|x\|^{2}\right)+u_{1, \kappa}\left(\|x\|^{2}\right) \hat{h}_{\bar{e}}(x /\|x\|) & \text { for } x \neq 0 \\ u_{2, \kappa}(0) & \text { for } x=0\end{cases}
$$

and

$$
h_{e_{\beta}^{k+2}}(x)= \begin{cases}u_{2, \beta}\left(\|x\|^{2}\right)+u_{1, \beta}\left(\|x\|^{2}\right) \hat{h}_{e_{\beta}^{k+2}}(x /\|x\|) & \text { for } x \neq 0 \\ u_{2, \beta}(0) & \text { for } x=0\end{cases}
$$

where on one hand smooth maps $u_{1, \kappa}, u_{1, \beta}$ are defined in the same way as $u_{1, \alpha}$ and, on the other hand, smooth maps $u_{2, \kappa}, u_{2, \beta}$ are similar to $u_{2, \alpha}$. Since $\left.h_{\tilde{e}}\right|_{S^{k+1}}=\left.\left(f^{k+1} \circ \Phi_{\tilde{e}}\right)\right|_{S^{k+1}}$ and $\left.h_{e_{\beta}^{k+2}}\right|_{S^{k+1}}=\left.\left(f^{k+1} \circ \Phi_{e_{\beta}^{k+2}}\right)\right|_{S^{k+1}}$, there are smooth maps $f_{\bar{e}}: \overline{\tilde{e}} \rightarrow \mathbb{R}$ and $f_{e_{\beta}^{k+2}}: \overline{e_{\beta}^{k+2}} \rightarrow \mathbb{R}$ such that

$$
\begin{aligned}
h_{\bar{e}} & =f_{\bar{e}} \circ \Phi_{\bar{e}} \\
\left.f_{\tilde{e}}\right|_{\partial \tilde{e}} & =\left.f^{k+1}\right|_{\partial \bar{e}} \\
h_{e_{\beta}^{k+2}} & =f_{e_{\beta}^{k+2}} \circ \Phi_{e_{\beta}^{k+2}} \\
\left.f_{e_{\beta}^{k+2}}\right|_{\partial e_{\beta}^{k+2}} & =\left.f^{k+1}\right|_{\partial e_{\beta}^{k+2}} .
\end{aligned}
$$

Let $\left\{\overline{e_{\gamma}^{k+2}}: \quad \gamma \in \Gamma\right\}$ be the closed $(k+2)$-cells containing each the closed cell $\overline{e^{*}}$, and let $\left\{\overline{e_{\beta, i}^{k+2}}: i \in I\right\}$ be the closed $(k+2)$-cells containing each the closed cell $\overline{e_{\alpha}^{k+1}}$. For each $\gamma \in \Gamma$, let

$$
\hat{h}_{e_{\gamma}^{k+2}}=\left.\left(f^{k+1} \circ \Phi_{e_{\gamma}^{k+2}}\right)\right|_{S^{k+1}},
$$

where $\Phi_{e_{\gamma}^{k+2}}$ is the characteristic map of the cell $e_{\gamma}^{k+2}$. And for each $i \in I$, let

$$
\hat{h}_{e_{\beta, i}^{k+2}}=\left.\left(f^{k+1} \circ \Phi_{e_{\beta, i}^{k+2}}\right)\right|_{S^{k+1}},
$$


where $\Phi_{e_{\beta, i}^{k+2}}$ is the characteristic map of the cell $e_{\beta, i}^{k+2}$. One constructs smooth maps $h_{e_{\gamma}^{k+2}}, h_{e_{\beta, i}^{k+2}}: D^{k+2} \rightarrow \mathbb{R}, f_{e_{\gamma}^{k+2}}: \overline{e_{\gamma}^{k+2}} \rightarrow \mathbb{R}$ and $f_{e_{\beta, i}^{k+2}}: \overline{e_{\beta, i}^{k+2}} \rightarrow \mathbb{R}$ such that

$$
\begin{aligned}
\left.h_{e_{\gamma}^{k+2}}\right|_{S^{k+1}} & =\left.\left(f^{k+1} \circ \Phi_{e_{\gamma}^{k+2}}\right)\right|_{S^{k+1}} \\
\left.h_{e_{\beta, i}^{k+2}}\right|_{S^{k+1}} & =\left.\left(f^{k+1} \circ \Phi_{e_{\beta, i}^{k+2}}\right)\right|_{S^{k+1}} \\
h_{e_{\gamma}^{k+2}} & =f_{e_{\gamma}^{k+2}} \circ \Phi_{e_{\gamma}^{k+2}} \\
\left.f_{e_{\gamma}^{k+2}}\right|_{\partial e_{\gamma}^{k+2}} & =\left.f^{k+1}\right|_{\partial e_{\gamma}^{k+2}} \\
h_{e_{\beta, i}^{k+2}} & =f_{e_{\beta, i}^{k+2}} \circ \Phi_{e_{\beta, i}^{k+2}} \\
\left.f_{e_{\beta, i}^{k+2}}\right|_{\partial e_{\beta, i}^{k+2}} & =\left.f^{k+1}\right|_{\partial e_{\beta, i}^{k+2}} .
\end{aligned}
$$

If $\left\{\overline{e_{\sigma}^{k+2}}: \sigma \in \Upsilon\right\}$ is the family of closed $(k+2)$-cells each of which does not contain neither $e^{*}$ nor $\overline{e_{\alpha}^{k+1}}$, define

$$
f_{e_{\sigma}^{k+2}}: \overline{e_{\sigma}^{k+2}} \rightarrow \mathbb{R}
$$

to be -1 .

Patching the $\left\{f_{e_{\gamma}^{k+2}}\right\}_{\gamma \in \Gamma},\left\{f_{e_{\beta, i}^{k+2}}\right\}_{i \in I},\left\{f_{e_{\sigma}^{k+2}}\right\}_{\sigma \in \Upsilon}, f_{\tilde{e}}, f_{e_{\beta}^{k+2}}$ and $f^{k+1}$ together one obtains a smooth map

$$
f^{k+2}: X^{(k+2)} \rightarrow \mathbb{R}
$$

such that

$$
p \in\left(f^{k+2}\right)^{-1}(0, \infty) \subset U \cap X^{(k+2)} .
$$

The map $f^{k+2}$ is clearly a smooth extension of $f^{k+1}$. 
Going one step up at a time one obtains for each $i \geq 0$ a smooth map $f^{k+i}: X^{(k+i)} \rightarrow \mathbb{R}$ such that $p \in\left(f^{k+i}\right)^{-1}(0, \infty) \subset U \cap X^{(k+i)} ;$ hence one is finished.

B) Now let us treat the case where the point $p$ is on the boundary of the cell $e^{k}$. Since $p$ is not a 0 -cell then it is a point of some cell $e^{l}(1 \leq l \leq k-1)$, with $e^{l} \subset \partial e^{k}$. As in case A), one constructs in the same way a sequence $\left\{f^{l+i}\right\}_{i \geq 0}$ of smooth maps $f^{l+i}: X^{(l+i)} \rightarrow \mathbb{R}$ such that $\left.f^{l+i+1}\right|_{X^{(l+1)}}=f^{(l+i)}$, $i \geq 0$ and $p \in\left(f^{l+i}\right)^{-1}(0, \infty) \subset U \cap X^{(l+i)}$. Afterwards define $f: X \rightarrow \mathbb{R}$ to be such that $\left.f\right|_{X^{(l+i)}}=f^{l+i}$; thus $p \in f^{-1}(0, \infty) \subset U$. The proof is thence complete. 
To complete the proof that the topology $\mathcal{T}$ is finer than the topology $\sigma$, we need to allow in Theorem 1.2.2 the point $p$ to be a 0 -cell of $X$.

Theorem 1.2.3 Let $(X, \mathcal{F})$ be a $D W$ complex and let $U$ be an open set in the topology $\sigma$. If $p$ is a 0 -cell in $X$ and an interior point of $U$, there exists a smooth map $f \in \mathcal{F}$ such that

$$
p \in f^{-1}(0, \infty) \subset U
$$

Proof. As in proof of Theorem 1.2.2, we will show that there are smooth maps $\left\{f^{i}: X^{(i)} \rightarrow \mathbb{R}\right\}_{i \geq 0}$ such that $\left.f^{i+1}\right|_{X^{(i)}}=f^{i}$ for each $i \geq 0$ and

$$
p \in\left(f^{i}\right)^{-1}(0, \infty) \subset U \cap X^{(i)} .
$$

Thus if $f: X \rightarrow \mathbb{R}$ is a function such that $\left.f\right|_{X^{(i)}}=f^{i}$, then $f$ is smooth and $p \in f^{-1}(0, \infty) \subset U$.

Assume $p=p_{j}$, where $p_{j} \in X^{(0)}$ and $X^{(0)}=\left\{p_{i}\right\}_{i \in I}$; the index set $I$ is possibly infinite. The function $f^{0}: X^{(0)} \rightarrow \mathbb{R}$ defined by

$$
f^{0}\left(p_{i}\right)= \begin{cases}1 & \text { if } i=j \\ -1 & \text { if } i \neq j\end{cases}
$$

is smooth and is such that

$$
\{p\}=\left(f^{0}\right)^{-1}(0, \infty) \subset U \cap X^{(0)} .
$$

If the 0 -cell $p$ is an isolated point of $X^{(1)}$, then Lemma 1.2.13 implies that it is an isolated point of $X$. Thus the function $f: X \rightarrow \mathbb{R}$ which takes on the 
value -1 at any other point of $X$ but is 1 at $p$ is smooth and is such that $p \in f^{-1}(0, \infty) \subset U$. So under such a circumstance, one is finished.

On the contrary, suppose that the 0 -cell $p$ is not an isolated point of $X^{(1)}$; hence $p$ is a boundary point of some closed one-cell $\bar{e}$. One may want to use the notation $e_{n}^{1}(p)$ to denote a $n$-th one-cell whose boundary contains the point $p$, but this notation is too cumbersome. We will try to avoid this notation, but in case we feel compelled to be more accurate we will use the notation $e^{1}(p)$ to refer to a one-cell bounded at least by $p$.

So in this case we are assuming that $p$ is a boundary point of some one-cell $e$. Suppose that $\bar{e}$ is a closed one-cell with $\Phi_{e}(-1)=p$ and $\Phi_{e}(1)=p_{l} \neq p$. Since $U \cap \bar{e}$ is open in $\bar{e}$ ( for the topology of $\mathcal{F}(\bar{e})$ ), there is an $\epsilon_{1} \in\left(0, \frac{1}{2}\right)$ such that

$$
\Phi_{e}\left(\left[-1,-1+\epsilon_{1}\right)\right) \subset U \cap \bar{e} .
$$

Choose a decreasing smooth $\operatorname{map} h_{e}^{1}:[-1,1] \rightarrow \mathbb{R}$ such that

$$
h_{e}^{1}(x)= \begin{cases}1 & \text { if } x=-1 \\ >0 & \text { if }-1 \leq x<-1+\frac{\epsilon_{1}}{2} \\ 0 & \text { if } x=-1+\frac{\epsilon_{1}}{2} \\ <0 & \text { if }-1+\frac{\epsilon_{1}}{2}<x \leq 1 \\ -1 & \text { if } x=1 .\end{cases}
$$

Since $\Phi_{e}:(-1,1) \rightarrow e$ is a diffeomorphism and $\Phi_{e}(-1) \neq \Phi_{e}(1)$, there is a smooth map $f_{e}^{1}: \bar{e} \rightarrow \mathbb{R}$ such that

$$
h_{e}^{1}=f_{e}^{1} \circ \Phi_{e}
$$


It is clear that

$$
\left(h_{e}^{1}\right)^{-1}(0, \infty) \subset \Phi_{e}^{-1}(U \cap \bar{e})
$$

and

$$
\operatorname{Fr}\left(\left(h_{e}^{1}\right)^{-1}(0, \infty)\right)<\operatorname{Fr}\left(\Phi_{e}^{-1}(U \cap \bar{e})\right)
$$

Therefore

$$
p \in\left(f_{e}^{1}\right)^{-1}(0, \infty) \subset U \cap \bar{e}
$$

and

$$
\operatorname{Fr}\left(f_{e}^{1}\right)^{-1}(0, \infty) \cap \operatorname{Fr}(U \cap \bar{e}) \cap e=\emptyset .
$$

The case where $\Phi_{e}(-1) \neq p$ and $\Phi_{e}(1)=p$ is treated in a similar manner. If both $\Phi_{e}(-1)$ and $\Phi_{e}(1)$ are not $p$, that is $\bar{e}$ is a closed one-cell not containing $p$, then define the corresponding $f_{e}^{1}$ to be the constant map -1 .

Suppose also there exists a one-cell $e$ such that $\Phi_{e}(-1)=\Phi_{e}(1)=p$. For such a cell $e$, we let the corresponding $h_{e}^{1}:[-1,1] \rightarrow \mathbb{R}$ to be a smooth map defined as follows

$$
h_{e}^{1}(x)= \begin{cases}1 & \text { if } x=-1 \text { or } x=1 \\ >0 & \text { if } x \in\left[-1,-1+\frac{\epsilon_{1}}{2}\right) \cup\left(1-\frac{\epsilon_{2}}{2}, 1\right] \\ 0 & \text { if } x=-1+\frac{\epsilon_{1}}{2} \text { or } x=1-\frac{\epsilon_{2}}{2} \\ <0 & \text { if } x \in\left(-1+\frac{\epsilon_{1}}{2}, 1-\frac{\epsilon_{2}}{2}\right),\end{cases}
$$

where $\epsilon_{1}$ and $\epsilon_{2}$ are suitably chosen in the interval $\left(0, \frac{1}{2}\right)$. Here as well, one obtains by virtue of Lemma 1.2 .2 a smooth map $f_{e}^{1}: \bar{e} \rightarrow \mathbb{R}$ such that $h_{e}^{1}=f_{e}^{1} \circ \Phi_{e}$. It is also clear that (1.3) and (1.4) are satisfied correspondingly. 
Denote by $X_{p}^{(1)}$ the family of those closed one-cells that have $p$ as a common boundary point. Consider the family $\left\{f_{e}^{1}: \bar{e} \in X_{p}^{1}\right\}$; one has $f_{e}^{1}(p)=1$ for all $\bar{e} \in X_{p}^{1}$. Since $f^{0}(p)=1$, we can patch the various $f_{e}^{1}$ with $f^{0}$ in order to define a smooth map $f^{1}: X^{(1)} \rightarrow \mathbb{R}$. Thus $\left(f^{1}\right)^{-1}(0, \infty)=$ $\left(f^{0}\right)^{-1}(0, \infty) \cup\left(\bigcup_{\bar{e} \in X_{p}^{1}}\left(f_{e}^{1}\right)^{-1}(0, \infty)\right)$, and hence

$$
p \in\left(f^{1}\right)^{-1}(0, \infty) \subset U \cap X^{(1)}
$$

as desired.

Now let us extend the map $f^{1}$ to the 2-skeleton $X^{(2)}$. The trivial case is when there is no closed 2-cell containing the 0 -cell $p$, in which case one defines $f: X \rightarrow \mathbb{R}$ to be a map given by

$$
f(x)= \begin{cases}f^{1}(x) & \text { for } x \in X^{(1)} \\ -1 & \text { otherwise. }\end{cases}
$$

It is clear that $f$ is smooth and $f^{-1}(0, \infty)=\left(f^{1}\right)^{-1}(0, \infty)$, therefore one gets $p \in f^{-1}(0, \infty) \subset U$.

On the other hand suppose that there is a family of closed 2-cells $\left\{\overline{e_{\alpha}^{2}}: \alpha \in \Lambda\right\}$ such that $p \in \partial e_{\alpha}^{2}$, for each $\alpha \in \Lambda$. For each $\alpha \in \Lambda$, define

$$
\hat{h}_{e_{\alpha}^{2}}=\left.\left(f^{1} \circ \Phi_{e_{\alpha}^{2}}\right)\right|_{S^{1}},
$$

where $\Phi_{e_{\alpha}^{2}}$ is the characteristic map of the cell $e_{\alpha}^{2}$. Since $\Phi_{e_{\alpha}^{2}}^{-1}(U)$ is open in $D^{2}$, there exists a set

$$
C_{\epsilon_{\alpha}}^{2}=\left\{x \in D^{2} \cap \Phi_{e_{\alpha}^{2}}^{-1}(U):\|x\|>1-\epsilon_{\alpha} \text { and } \hat{h}_{e_{\alpha}^{2}}(x /\|x\|)>0\right\}
$$

for some sufficiently small $\epsilon_{\alpha}$ such that

$$
\operatorname{Fr} C_{\epsilon_{\alpha}}^{2} \cap \operatorname{Fr} \Phi_{e_{\alpha}^{2}}^{-1}(U) \cap \operatorname{int} D^{2}=\emptyset .
$$


From this stage one repeats mutatis mutandis the proof of Theorem 1.2.2 to extend $f^{1}$ to some smooth $f^{2}$, and $f^{2}$ to some smooth $f^{3}$, and so on ..., but every time one has $p \in\left(f^{i}\right)^{-1}(0, \infty) \subset U \cap X^{(i)}$.

We, finally, need to prove the reverse inclusion $\mathcal{T} \subset \sigma$ to corroborate the statement that the underlying topological space of a DW complex is a $\mathrm{CW}$ complex.

Theorem 1.2.4 Let $(X, \mathcal{F})$ be a $D W$ complex with the topology induced by $\mathcal{F}$. If $\sigma$ is the topology on $X$ such that $U$ is in $\sigma$ if and only if $U \cap \bar{e}$ is open with respect to the differential structure $\mathcal{F}(\bar{e})$, then $\mathcal{T} \subset \sigma$.

Proof. Let $U$ be an open set of $X$ with respect to $\mathcal{T}$, and let $p \in U \cap \bar{e}$, where $e$ is a cell of $X$. Then there is a map $f \in \mathcal{F}$ such that $p \in f^{-1}(0, \infty) \subset U$. But $\left.f\right|_{\bar{e}} \in \mathcal{F}(\bar{e})$, therefore $p \in\left(\left.f\right|_{\bar{e}}\right)^{-1}(0, \infty) \subset U \cap \bar{e}$. Thus $U \cap \bar{e}$ is open in $\bar{e}$, and hence $U$ is in $\sigma$.

Putting Theorems 1.2.2, 1.2.3 and 1.2.4 together implies

Theorem 1.2.5 The underlying space of a $D W$ complex is a $C W$ complex.

Because the underlying topology of a DW complex confers a CW structure on the underlying set, DW complexes are given 'freely', so to speak, many nice properties of $\mathrm{CW}$ complexes, among which we list the following without proof: 
(i) A subset $A$ of a DW complex $X$ is closed if and only if $A \cap Y$ is closed in $Y$ for every finite DW subcomplex $Y$ in $X$.

(ii) A subset $Y=\cup\left\{e: e \in E^{\prime}\right\}$ of a DW complex $(X, E), E^{\prime} \subset E$, is a DW subcomplex if and only if $Y$ is closed.

(iii) Every cell $e^{n}$ is an open subset of the skeleton $X^{(n)}$.

(iv) The compiement of $X^{(n-1)}$ in $X^{(n)}$ is an open subset of $X^{(n)}$.

(v) Every path component of a DW complex is a DW subcomplex.

(vi) A DW complex is connected if and only if it is path connected.

(vii) Every DW complex $X$ is a normal space.

(viii) A compact subset of a DW complex lies in a finite DW subcomplex. Therefore, a DW complex is compact if and only if it is finite.

For proofs of the above assertions, the reader is referred to [11], [14], [18] .

If $X$ is a CW complex and $Y$ is a CW subcomplex of $X$, it is known that the quotient space $X / Y$ is a CW complex, see [[14], p.280] or [[18], p.208] . One would be tempted to find out whether the same result holds in the setting of DW complexes. Instead of tackling the question as it is posed in the category of $\mathrm{CW}$ complexes, we will rather attend to a particular problem.

As a requirement to the remainder of Chapter 1, we assume knowledge of supports of functions and of locally finite open refinements of coverings. Let 
us briefly recall that if $f$ is a real-valued function then the support of $f$, denoted $\operatorname{supp}(f)$, is the closure of $\{x \mid f(x) \neq 0\}$.

Let $\mathcal{U}=\left\{U_{i}: i \in I\right\}$ and $\mathcal{V}=\left\{V_{j}: j \in J\right\}$ be open coverings of a topological space $X$. The covering $\mathcal{V}$ is said to be a locally finite refinement of $\mathcal{U}$ if each $V_{j}$ is contained in some $U_{i}$ of $\mathcal{U}$ and each point $x \in X$ has a neighbourhood that meets finitely many members of $\mathcal{V}$.

Definition [9] Let $\mathcal{U}=\left\{U_{i}: i \in I\right\}$ be an open covering of a differential space $X$. Then a smooth partition of unity subordinate to $\mathcal{U}$ is a collection $\left\{f_{j}\right\}_{j \in J}$ of smooth maps $f_{j}: X \rightarrow[0,1]$ having the following properties:

(i) There is a locally finite open refinement $\left\{V_{j}: j \in J\right\}$ of $\mathcal{U}$ such that $\operatorname{supp}\left(f_{j}\right) \subset V_{j}$ for all $j \in J$,

(ii) $\sum_{j} f_{j}(x)=1$ for each $x \in X$.

Lemma 1.2.14 Let $X$ be a topological space, $\mathcal{U}=\left\{U_{i} \mid i \in I\right\}$ an open covering of $X$ and $A$ a subset of $X$. Then $\mathcal{U} \cap A$ is an open covering of $A$; moreover if $\mathcal{V}=\left\{V_{j} \mid j \in J\right\}$ is a locally finite open refinement of $\mathcal{U}$ then $\mathcal{V} \cap A$ is a locally finite open refinement of $\mathcal{U} \cap A$.

The proof is straightforward.

Lemma 1.2.15 If $X$ is a differential space such that every open covering has a subordinate smooth partition of unity, then every open covering of any closed subspace $A$ of $X$ has a smooth partition of unity. 
Proof. Let $\mathcal{U}^{\prime}$ be an open covering of $A$, where $A$ is closed in $X$. Then there is an open covering $\mathcal{U}$ of $X$ such that $\mathcal{U}^{\prime}=\mathcal{U} \cap A$. If $\left\{f_{j}\right\}_{j \in J}$ is a smooth partition of unity subordinate to $\mathcal{U}$ and $\mathcal{V}=\left\{V_{j} \mid j \in J\right\}$ is a locally finite open refinement of $\mathcal{U}$ such that $\operatorname{supp}\left(f_{j}\right) \subset V_{j}$ for all $j \in J$, then $\operatorname{supp}\left(\left.f_{j}\right|_{A}\right) \subset V_{j} \cap A$ for all $j \in J$, and $\mathcal{V} \cap A=\left\{V_{j} \cap A \mid j \in J\right\}$ is a locally finite refinement of $\mathcal{U}^{\prime}$, by Lemma 1.2.14. Since $\sum_{j} f_{j}(x)=1$ for each $x \in X$, it follows that $\left\{\left.f_{j}\right|_{A}\right\}_{j \in J}$ is a smooth partition of unity subordinate to $\mathcal{U}^{\prime}$.

Definition A handy differential space is a differential space which satisfies the following property: every open covering has a smooth partition of unity.

Lemma 1.2.16 If $(X, \mathcal{F})$ is a coproduct of a family $\left\{\left(X_{i}, \mathcal{F}_{i}\right) \mid i \in I\right\}$ of handy differential spaces, then $(X, \mathcal{F})$ is a handy differential space.

Proof. Let $\mathcal{U}$ be an open covering of $X$. Since $X=\sqcup X_{i}$, Lemma 1.2.14 implies that each $\mathcal{U} \cap X_{i}$ is an open covering for the corresponding $X_{i}$. But $X_{i}$ is a handy differential space, therefore there is a smooth partition of unity $\left\{f_{j}\right\}_{j \in J_{i}}$ subordinate to $\mathcal{U} \cap X_{i}$. Suppose that $\mathcal{V}_{i}=\left\{V_{j}: j \in J_{i}\right\}$ is a locally finite open refinement of $\mathcal{U} \cap X_{i}$ such that $\operatorname{supp}\left(f_{j}\right) \subset V_{j}$ for all $j \in J_{i}$. It is easy to see that $\cup_{i \in I} \mathcal{V}_{i}$ is a locally finite open refinement of $\mathcal{U}$.

Now define for each $i \in I$ and each $j \in J_{i}$

$$
g_{j i}(x)= \begin{cases}f_{j}(x) & \text { for } x \in X_{i} \\ 0 & \text { for } x \notin X_{i} .\end{cases}
$$

Clearly, every $g_{j i}$ is smooth on $X$ and

$$
\left\{x \in X \mid g_{j i}(x) \neq 0\right\}=\left\{x \in X_{i} \mid f_{j}(x) \neq 0\right\} .
$$


Since $X_{i}$ is both open and closed in the coproduct $X$, it follows that $\operatorname{supp}\left(g_{j i}\right)=$ $\operatorname{supp}\left(f_{j}\right)$ for all $j \in J_{i}$. But $\operatorname{supp}\left(f_{j}\right) \subset V_{j}$ where $V_{j} \in \mathcal{V}_{i}$, so there exists an open neighbourhood $W_{j}=V_{j} \in \cup \mathcal{V}_{i}$ such that $\operatorname{supp}\left(g_{j i}\right) \subset W_{j}$.

Moreover, observe that

$$
\sum_{i} \sum_{j} g_{j i}(x)=\sum_{j} f_{j}(x)=1
$$

for each $x \in X$; our proof is thus complete.

Definition A handy DW complex is a DW complex whose underlying space is a handy differential space.

In [9], it is shown that every open covering of a $C^{\infty}$-manifold has a subordinate smooth partition of unity. One of the simplest examples of a handy differential space is the euclidean differential space $\mathbb{R}^{n}$.

Definition [22] Recall that a non-empty differential subspace $\left(A, \mathcal{F}_{A}\right)$ of a differential space $(X, \mathcal{F})$ is said to have the global extension property in $(X, \mathcal{F})$ if every $f \in \mathcal{F}_{A}$ can be extended to some smooth function $\bar{f}$ on the whole of $X$, that is for every function $f \in \mathcal{F}_{A}$ there is a function $\bar{f} \in \mathcal{F}$ such that $f=\left.\bar{f}\right|_{A}$.

The following lemma shows a condition under which a non-empty closed subspace of a differential space has the global extension property.

Lemma 1.2.17 If $(X, \mathcal{F})$ is a differential space with smooth partitions of unity, then every non-empty closed subspace $\left(A, \mathcal{F}_{A}\right)$ has the global extension 
property in $(X, \mathcal{F})$.

Proof. See [22].

Theorem 1.2.6 Let $(X, \mathcal{F})$ be a handy $D W$ complex whose underlying space $X$ is regular, let $Y$ be a compact $D W$ subcomplex, and let $\nu: X \rightarrow X / Y$ be the natural map. If $\nu^{*}(\mathcal{F})$ is the coinduced differential structure on $X / Y$, corresponding to $\nu$, then $\left(X / Y, \nu^{*}(\mathcal{F})\right)$ is a $D W$ complex.

Proof. Let us first prove that the restriction

$$
\left.\nu\right|_{X-Y}:(X-Y, \mathcal{G}) \rightarrow(X / Y-\{*\}, \mathcal{H})
$$

where $\mathcal{G}=\mathcal{F}_{X-Y}$ and $\mathcal{H}=\nu^{*}(\mathcal{F})_{X / Y-\{*\}}$, is a diffeomorphism. It is plain that $\mathcal{H}$ is generated by functions $g: X / Y-\{*\} \rightarrow \mathbb{R}$, where $g=\left.f\right|_{X / Y-\{*\}}$ for some $f \in \nu^{*}(\mathcal{F})$. For every $f \in \nu^{*}(\mathcal{F})$, one has

$$
\left.\left.f\right|_{X / Y-\{*\}} \circ \nu\right|_{X-Y} \in \mathcal{G}
$$

By Lemma 1.1.5, it follows that $\left.\nu\right|_{X-Y}$ is smooth. But to show that $\left(\left.\nu\right|_{X-Y}\right)^{-1}$ : $(X / Y-\{*\}, \mathcal{H}) \rightarrow(X-Y, \mathcal{G})$ is a little bit tricky.

Let $p$ be any point in $X-Y$. Since $X$ is Hausdorff and $Y$ is compact by hypothesis, there exists an open set $N$ containing $p$ such that $N \cap Y=\emptyset$. Using regularity of $X$, one can assume that for each $q \in Y$ there exist open neighbourhoods $V_{q}$ and $U_{q}$ of $p$ and $q$ respectively such that $\bar{N} \subset V_{q}$ and $V_{q} \cap U_{q}=\emptyset$. Since $Y$ is compact, there are finitely many points $q_{1}, q_{2}, \ldots, q_{n} \in Y$ such 
that $Y \subset \cup_{i=1}^{n} U_{q_{i}}$. But every $U_{q_{\mathrm{i}}} \cap V_{q_{\mathrm{i}}}=\emptyset$, with $V_{q_{\mathrm{i}}}$ an open neighbourhood of $\bar{N}$. Therefore, if we put $U=\cup_{i=1}^{n} U_{q_{i}}$ and $V=\cap_{i=1}^{n} V_{q_{i}}$, then $U$ and $V$ are open sets such that $Y \subset U, p \in \bar{N} \subset V$ and $U \cap V=\emptyset$. Since $X$ is Hausdorff, it follows that the subset $X-Y-\bar{N}$ is open in $X$ and so the family

$$
\mathcal{C}=\{U, V, X-Y-\bar{N}\}
$$

is an open covering of $X$. We know that $X$ is a handy DW complex. Therefore, let $\mathcal{W}=\left\{W_{j}\right\}_{j \in J}$ be a locally finite open refinement of $\mathcal{C}$. There exist only finitely many $j_{1}, \ldots, j_{n} \in J$ such that $p \in W_{j_{i}}, i=1, \ldots, n$. It is clear that $W_{j_{i}} \subset V$ for each $i=1, \ldots, n$.

Now, let $J_{1}=\left\{j \in J: W_{j} \subset V\right\}$ and $J_{2}=J-J_{1}$. Let $f=\left.h\right|_{X-Y}$, where $h \in \mathcal{F}$. For each $j \in J$, let $g_{j}: X \rightarrow \mathbb{R}$ be a function given as follows: (1) if $j \in J_{1}$,

$$
g_{j}(x)= \begin{cases}f(x) & \text { if } x \in W_{j} \\ 0 & \text { otherwise }\end{cases}
$$

(2) if $j \in J_{2}$,

$$
g_{j}=0 \text {. }
$$

Let $\left\{\lambda_{j}\right\}_{j \in J}$ be a partition of unity subordinate to $\mathcal{W}$, and let

$$
f^{*}=\sum_{j \in J} \lambda_{j} g_{j}
$$

We claim that $f^{*}$ is smooth. Indeed, one can write

$$
f^{*}=\sum_{j \in J_{1}} \lambda_{j} g_{j}+\sum_{j \in J_{2}} \lambda_{j} g_{j}
$$


For each $j \in J_{2}, \lambda_{j} g_{j}=0$. Hence for each $j \in J_{2}, \lambda_{j} g_{j}$ is smooth on $X$. Let $j \in J_{1}$. To see that $\lambda_{j} g_{j}$ is smooth, one need only look at points on the boundary $\operatorname{Fr} W_{j}$ of $W_{j}$. Since $W_{j} \subset V$ and $U \cap V=\emptyset$, it follows that $Y \cap \operatorname{Fr} W_{j}=\emptyset$. One can convince oneself of this fact by assuming that there is a point $x \in Y \cap \operatorname{Fr} W_{j}$. Then every open neighbourhood of $x$ meets $Y$ and $W_{j}$, and indirectly meets $U$ and $V$, because $W_{j} \subset V$. But $U$ is an open neighbourhood of $x$ and $U \cap V=\emptyset$. Therefore the assumption that $Y \cap \operatorname{Fr} W_{j} \neq \emptyset$ doesn't hold. Let $r \in \operatorname{Fr} W_{j}$. As $Y$ is closed, there exists an open neighbourhood $R \subset X-Y$ of $r$ such that

$$
\left(\lambda_{j} g_{j}\right)(t)= \begin{cases}\lambda_{j}(t) f(t) & \text { if } t \in R \cap W_{j} \\ 0 & \text { if } t \in R-W_{j} .\end{cases}
$$

Since $\lambda_{j}(t) f(t)=0$ for all $t \in R-W_{j}$, so $\lambda_{j} g_{j}=\lambda_{j} f$ on $R$ and hence $\lambda_{j} g_{j}$ is smooth on $R$. Thus, $f^{*}$ is a smooth function.

Put

$$
\mu_{j}=\sum_{j \in J_{1}} \lambda_{j} g_{j} \quad \text { and } \quad \delta_{j}=\sum_{j \in J_{2}} \lambda_{j} g_{j} .
$$

Since $\left.\lambda_{j}\right|_{X-W_{j}}=0$ and $Y \subset X-\cup_{j \in J_{1}} W_{j}$, it follows that

$$
\left.\mu_{j}\right|_{Y}=0 .
$$

Since $g_{j}=0$ on $N_{j}$, where $j \in J_{2}$, it follows that

$$
\left.\delta_{j}\right|_{Y}=0 .
$$

Thus,

$$
\left.f^{*}\right|_{Y}=0 .
$$


Now, let $W^{*}=\cap_{i=1}^{n} W_{j_{i}} \cap N$. It is evident that $W^{*}$ is an open neighbourhood of $p$ and is contained in $X-Y$. Consider,

$$
\left.f^{*}\right|_{W^{*}}=\sum_{j \in J} \lambda_{\left.j\right|_{W}} g_{\left.j\right|_{W^{*}}}
$$

It follows that

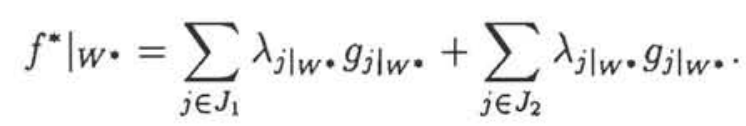

Let $l \in J_{2}$. Therefore, $W_{l}$ is either contained in $U$ or in $X-Y-\bar{N}$. Thus, $\left.\lambda_{l}\right|_{N}=0$ and hence $\lambda_{\left.l\right|_{W}}=0$. Thus,

$$
\begin{aligned}
\left.f^{*}\right|_{W} & =\sum_{j \in J_{1}} \lambda_{\left.j\right|_{W}} g_{\left.j\right|_{W} \cdot} \\
& =\left.\left.\left(\sum_{j \in J_{1}} \lambda_{j}\right)\right|_{W} \cdot f\right|_{W^{*}} \\
& =\left.f\right|_{W^{*}}
\end{aligned}
$$

It follows that

$$
\left.\left.f^{*}\right|_{W} \cdot \circ \nu^{-1}\right|_{\nu\left(W^{*}\right)}=\left.\left.f\right|_{W} \circ \nu^{-1}\right|_{\nu\left(W^{*}\right)}
$$

We claim that $f^{*} \circ \nu^{-1}: X / Y \rightarrow \mathbb{R}$ is smooth. Indeed, in the diagram

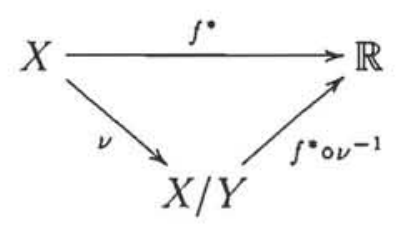

the smooth map $f^{*}: X \rightarrow \mathbb{R}$ is constant on every $\nu^{-1}(u)$, where $u \in X / Y$. Therefore, one applies Corollary 1.1 .2 at once to show that $f^{*} \circ \nu^{-1}: X / Y \rightarrow$ $\mathbb{R}$ is smooth. 
Now, since $W^{*}$ is an open neighbourhood of $p$, then by Lemma 1.1.7, it follows that there exists a nonnegative function $h^{*} \in \mathcal{F}$ such that $p \in h^{*-1}(0, \infty) \subset$ $W^{*}$, and thus $Y \subset\left(h^{*}\right)^{-1}(0)$. Since $h^{*}: X \rightarrow \mathbb{R}$ is constant on every $\nu^{-1}(u)$, where $u \in X / Y$, Corollary 1.1.2 applies to show that $h^{*} \circ \nu^{-1}: X / Y \rightarrow \mathbb{R}$ is smooth. It is clear that $\nu(p) \in\left(h^{*} \circ \nu^{-1}\right)^{-1}(0, \infty) \subset \nu\left(W^{*}\right)$. But $p$ is arbitrary in $W^{*}$, therefore $\nu\left(W^{*}\right)$ is an open subset of $X / Y$. So we have an open subset $\nu\left(W^{*}\right)$, a smooth map $f^{*} \circ \nu^{-1}: X / Y \rightarrow \mathbb{R}$ such that

$$
\left.\left(f^{*} \circ \nu^{-1}\right)\right|_{\nu(W \cdot)}=\left.\left(f \circ \nu^{-1}\right)\right|_{\nu(W \cdot)} .
$$

Thus, $\left.f \circ \nu^{-1}\right|_{X / Y-\{*\}}: X / Y-\{*\} \rightarrow \mathbb{R}$ is locally smooth, and consequently $\left.f \circ \nu^{-1}\right|_{X / Y-\{*\}}$ is smooth. Hence, by Lemma 1.1.5, $\left.\nu^{-1}\right|_{X / Y-\{*\}}: X / Y-$ $\{*\} \rightarrow X-Y$ is smooth.

Finally, let us prove that $\left(X / Y, \nu^{*}(\mathcal{F})\right)$ is a DW complex.

First let us prove that $X / Y$ is Hausdorff. Let $\nu(x), \nu(z)$ be distinct points in $X / Y$. Assume that $\nu(x) \neq *$ and $\nu(z) \neq *$. Therefore $x \notin Y$ and $z \notin Y$; since $X$ is Hausdorff and $X-Y$ is open, there are disjoint open subsets $U^{\prime}$ and $V^{\prime}$ in $X-Y$ such that $x \in U^{\prime}$ and $z \in V^{\prime}$. But $X-Y$ is diffeomorphic to $X / Y-\{*\}$, therefore $\nu\left(U^{\prime}\right)$ and $\nu\left(V^{\prime}\right)$ are open neighbourhoods of $\nu(x)$ and $\nu(z)$ respectively and such that $\nu\left(U^{\prime}\right) \cap \nu\left(V^{\prime}\right)=\emptyset$.

Now assume that $\nu(z)=*$. Therefore $x \notin Y$; since $X$ is Hausdorff and $Y$ is compact, there are disjoint open subsets $U^{\prime \prime}$ and $V^{\prime \prime}$ in $X$ such that $x \in U^{\prime \prime}$ and $Y \subset V^{\prime \prime}$. Let

$$
\mathcal{R}=\left\{U^{\prime \prime}, V^{\prime \prime}, X-Y\right\}
$$


be an open covering of $X$. The space $X$ is a handy DW complex; it follows that $\mathcal{R}$ has a locally finite open refinement, say $\mathcal{W}^{\prime}=\left\{W_{i}^{\prime}\right\}_{i \in I}$. Let $I_{1}=\{i \in$ $\left.I: W_{i}^{\prime} \cap Y=\emptyset\right\}$ and let $I_{2}=\left\{i \in I: W_{i}^{\prime} \cap Y \neq \emptyset\right\}$. Next, for each $i \in I$, let $h_{i}: X \rightarrow \mathbb{R}$ be a function defined by

$$
h_{i}=\left\{\begin{array}{ll}
0 & \text { if } i \in I_{1} \\
1 & \text { if } i \in I_{2}
\end{array} .\right.
$$

Let $\left\{\mu_{i}\right\}_{i \in I}$ be a partition of unity subordinate to $\mathcal{W}^{\prime}$, and let

$$
r=\sum_{i \in I} \mu_{i} h_{i}
$$

It is easy to see that the map $r: X \rightarrow \mathbb{R}$ is smooth on $X$. Put

$$
A=\sum_{i \in I_{1}} \mu_{i} h_{i} \quad \text { and } \quad B=\sum_{i \in I_{2}} \mu_{i} h_{i}
$$

Clearly, one has

$$
\left.A\right|_{V^{\prime \prime}}=0 \quad \text { and }\left.B\right|_{V^{\prime \prime}}=\left.\sum_{i \in I_{2}} \mu_{i}\right|_{V^{\prime \prime}} .
$$

Thus,

$$
\left.r\right|_{V^{\prime \prime}}=\left.B\right|_{V^{\prime \prime}} .
$$

Note that

$$
\left.r\right|_{Y}=1
$$

and

$$
Y \subset r^{-1}(0, \infty) \subset V^{\prime \prime}
$$


Since $r$ is constant on each $\nu^{-1}(u), u \in X / Y$, Corollary 1.1.2 says that $r$ induces a smooth map $r^{\prime}: X / Y \rightarrow \mathbb{R}$ such that $* \in\left(r^{\prime}\right)^{-1}(0, \infty)$. Finally, since $\left.\nu\right|_{X-Y}: X-Y \rightarrow X / Y-\{*\}$ is a diffeomorphism, it follows that $\nu\left(U^{\prime \prime}\right)$ is an open neighbourhood of $\nu(x)$. Moreover since $U^{\prime \prime} \cap r^{-1}(0, \infty)=\emptyset$, it follows that $\nu\left(U^{\prime \prime}\right) \cap\left(r^{\prime}\right)^{-1}(0, \infty)=\emptyset$. Hence, $X / Y$ is Hausdorff.

Let $E(X)$ and $E(Y)$ be the families of cells of $X$ and $Y$ respectively. We define cells in $X / Y$ as follows. The 0 -cells are given by

$$
(X / Y)^{0}=\{\nu(e): e \in E(X)-E(Y) \text { and } \operatorname{dim}(e)=0\} \cup\{*\} ;
$$

for $k>0$, define

$$
(X / Y)^{k}=\{\nu(e): e \in E(X)-E(Y) \text { and } \operatorname{dim}(e)=k\} .
$$

If $(X / Y)^{(k)}$ is the union of all cells of dimension $\leq k$, then $(X / Y)^{(k)}=$ $\cup_{i=0}^{k}(X / Y)^{i}$. Let $e$ be a cell in $X-Y$; we define the characteristic map of $\nu(e)$ as the composite $\nu \Phi_{e}$. The characteristic map of the cell $*$ is defined to be the map $\Phi_{*}: D^{0} \rightarrow *$.

We now prove that $X / Y$ satisfies all the 4 axioms defining a DW complex.

(1d) $X / Y$ is the disjoint union of its cells. Indeed, the reason is that $X-Y$ is a disjoint union of cells, $X-Y$ is diffeomorphic to $X / Y-\{*\}$ and $\nu(Y)=\{*\}$.

(2d) Let $k>0$. If $e$ is a $k$-cell in $X-Y$, then $\overline{\nu(e)}=\nu \Phi_{e}\left(D^{k}\right)=\nu(\bar{e})$. Since $\Phi_{e}$ and $\nu$ are quotients, it follows that $\nu \Phi_{e}: D^{k} \rightarrow \overline{\nu(e)}$ is quotient. Since $\left.\nu\right|_{X-Y}: X-Y \rightarrow X / Y-\{*\}$ is a diffeomorphism, then 
Lemma 1.1.6 implies that each $\left.\nu\right|_{e}: e \rightarrow \nu(e), e \in E(X)-E(Y)$, is a diffeomorphism. But $\left.\Phi_{e}\right|_{\check{D}^{k}}: \breve{D}^{k} \rightarrow e$, where $e$ is a $k$-cell in $X-Y$, is a diffeomorphism, therefore $\left.\nu \Phi_{e}\right|_{\breve{D}^{k}}: \breve{D}^{k} \rightarrow \nu(e)$ is a diffeomorphism. The case $e=*$ is trivial. Hence, (2d) holds.

(3d) Let $e$ be a cell in $X-Y$. Since $\bar{e}$ is a union of finitely many cells in $X$, and since $\left.\nu\right|_{X-Y}: X-Y \rightarrow X / Y-\{*\}$ is a diffeomorphism, it follows that $\overline{\nu(e)}$ is also some finite union of cells in $X / Y$. The cells in $Y$ end up under $\nu$ as $*$.

(4d) Let us now show that $\nu^{*}(\mathcal{F})$ is in fact the structure coinduced on $X / Y$ by the inclusions $\overline{\nu(e)} \rightarrow X / Y$, where $e$ is a cell in $X$. Given $f \in \nu^{*}(\mathcal{F})$, we have the following implications: $f \in \nu^{*}(\mathcal{F})$ if and only if $f \circ \nu \in \mathcal{F}$. But $f \circ \nu \in \mathcal{F}$ if and only if $\left.\left.f\right|_{\overline{\nu(e)}} \circ \nu\right|_{\bar{e}} \in \mathcal{F}(\bar{e})$ for each $e \in E$, that is if and only if $\left.f\right|_{\overline{\nu(e)}}: \overline{\nu(e)} \rightarrow \mathbb{R}$, where $e$ is a cell of $E$, is smooth. Thus, a map $f: X / Y \rightarrow \mathbb{R}$ is in $\nu^{*}(\mathcal{F})$ if and only if $\left.f\right|_{\overline{\nu(e)}}, e \in E$ is smooth, as desired. 


\section{Chapter 2}

\section{ALTERNATIVE \\ DESCRIPTION OF A DW COMPLEX}

This chapter shows how one can build up a DW complex by means of cells as in case of $\mathrm{CW}$ complexes. To define a $\mathrm{CW}$ complex in terms of attaching cells, one needs continuous maps to be able to attach cells to each other; whereas for a DW complex one requires smooth maps. These smooth maps are called attaching maps or characteristic maps. The attaching of cells is done progressively with respect to the dimensions of cells. More precisely, let $X^{(0)}$ be a discrete set of points. We call these points the 0 -cells. Then we attach the 1-sells to $X^{(0)}$ to get $X^{(1)}$; afterwards we attach the 2-cells to $X^{(1)}$, and so on. 
We will prove that this way of defining DW complexes is equivalent to the intrinsic definition of Chapter 1 only for finite DW complexes.

In section 2 of this chapter, we will introduce the class of FW complexes. FW complexes are meant to be analogues of CW complexes in the category of Frölicher spaces. We will show that for any FW complex if one leaves aside the curves, one ends up with a DW complex. And we will also prove that if $(X, \mathcal{F}, \mathcal{C})$ is a Frölicher space whose underlying differential space $(X, \mathcal{F})$ is a DW complex, then $(X, \mathcal{F}, \mathcal{C})$ is a $\mathrm{FW}$ complex with respect to the same collections of cells and characteristic maps of the DW complex $(X, \mathcal{F})$.

\subsection{Building up a DW complex}

First we begin by recalling the construction of an adjunction space of two topological spaces.

Definition Let $X$ and $Y$ be disjoint topological spaces, let $A$ be a closed subset of $X$, and let $f: A \rightarrow Y$ be a continuous map. The adjunction space $X \sqcup_{f} Y$ is the space $(X \sqcup Y) / \sim$, where $\sim$ is the smallest equivalence relation identifying $a \in A$ with its image $f(a) \in Y$.

If we let $\nu: X \sqcup Y \rightarrow X \sqcup_{f} Y$ be the quotient map, then $\left.\nu\right|_{Y}: Y \rightarrow X \sqcup_{f} Y$ is a homeomorphism of $Y$ onto $\nu(Y)$, and $\left.\nu\right|_{X-A}: X-A \rightarrow X \sqcup_{f} Y$ is a homeomorphism of $X-A$ onto $\nu(X-A)$. See [18] or [11]. 
Definition Let $X$ and $Y$ be differential spaces, and let $f: A \rightarrow Y$ be a smooth map, where $A$ is a non-empty closed subspace of $X$. Consider the coproduct $X \sqcup Y$ and form a quotient space by identifying each set

$$
\{y\} \cup f^{-1}(y),
$$

for $y \in Y$, to a point. We denote this quotient space by $X \sqcup_{f} Y$ and call it an adjunction space of $X$ and $Y$ determined by $f$ or the differential space obtained from $Y$ by attaching (or by gluing) $X$ via $f$.

Unless specified otherwise, if $X \sqcup_{f} Y$ is the adjunction of differential spaces $X$ and $Y$, we will assume that $X$ is a handy differential space.

Lemma 2.1.1 Let $X \sqcup_{f} Y$ be an adjunction space, and $f: A \rightarrow Y$ is a smooth map carrying a closed subspace $A$ of $X$ into the differential space $Y$. If $\nu: X \sqcup Y \rightarrow X \sqcup_{f} Y$ is the quotient map such that each $a \in A$ with its image $f(a)$ collapse to a point, then the relation $u \sim v, u, v \in X \sqcup Y$, if and only if $\nu(u)=\nu(v)$ is an equivalence relation on $X \sqcup Y$.

Proof. Easy to check.

With the same notation of Lemma 2.1.1, we have

Lemma 2.1.2 Let $W$ be a differential space for which there exists a smooth surjective map $h: X \sqcup Y \rightarrow W$ such that, for $u, v \in X \cup Y$, one has $\nu(u)=\nu(v)$ if and only if $h(u)=h(v)$. Then $[u] \mapsto h(u)$ is a diffeomorphism $X \sqcup_{f} Y \rightarrow W$. Thus, $X \sqcup_{f} Y$ is unique up to a diffeomorphism. 
Proof. Consider the diagram

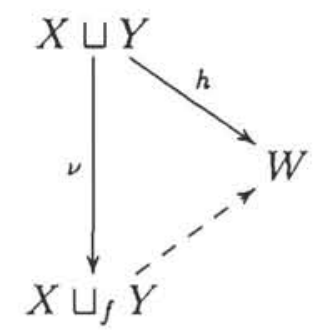

By hypothesis, the equivalence relation $u \sim v$ if and only if $\nu(u)=\nu(v)$, $u, v \in X \sqcup Y$, coincides with the equivalence relation Kerh. Thus, $X \sqcup_{f} Y=$ $(X \sqcup Y) /_{\text {Kerh }}$, and Corollary 1.1.3 applies at once to show that the dashed arrow $[u] \mapsto h(u)$ is a diffeomorphism.

Remark If $\mathcal{F}$ is the differential structure of $X$ and $\mathcal{G}$ the differential structure of $Y$, we shall denote by $\mathcal{F} \cup_{f} \mathcal{G}$ the differential structure on the adjunction $X \sqcup_{f} Y$.

If $\nu: X \sqcup Y \rightarrow X \sqcup_{f} Y$ is the quotient map, then $\left.\nu\right|_{Y}: Y \rightarrow \nu(Y)$ (resp. $\left.\nu\right|_{X-A}: X-A \rightarrow \nu(X-A)$ ) maps the set $Y$ (resp. $X-A$ ) bijectively onto $\nu(Y)$ (resp. $\nu(X-A)$ ). As we now show: $\left.\nu\right|_{Y}$ and $\left.\nu\right|_{X-A}$ are actually diffeomorphisms.

Lemma 2.1.3 Let $\left(X \sqcup_{f} Y, \mathcal{F} \sqcup_{f} \mathcal{G}\right)$ be the adjunction space of differential spaces $(X, \mathcal{F})$ and $(Y, \mathcal{G})$ by the smooth map $f: A \rightarrow Y$, where $A$ is closed in $X$. Let $\mathcal{C}:=\mathcal{F} \sqcup_{f} \mathcal{G}$. If $\nu: X \sqcup Y \rightarrow X \sqcup_{f} Y$ is the quotient map, then any function $\alpha: \nu(Y) \rightarrow \mathbb{R}$ such that $\left.\alpha \circ \nu\right|_{Y} \in \mathcal{G}$ is a structure map for the induced differential structure $\mathcal{C}_{\nu(Y)}$. 
Proof. First note that $\left.\alpha \circ \nu\right|_{A}=\left.\alpha \circ \nu\right|_{Y} \circ \iota_{Y} \circ f$, where $\iota_{Y}: Y \rightarrow X \sqcup Y$ is an inclusion. Since, by hypothesis, $\left.\alpha \circ \nu\right|_{Y} \in \mathcal{G}$, it follows that $\left.\alpha \circ \nu\right|_{Y} \circ \iota_{Y} \circ f \in \mathcal{F}_{A}$, and thus $\left.\alpha \circ \nu\right|_{A} \in \mathcal{F}_{A}$. By Lemma 1.2.17, there exists $g \in \mathcal{F}$ such that $\left.g\right|_{A}=\left.\alpha \circ \nu\right|_{A}$. Now, let $\beta: \nu(X-A) \rightarrow \mathbb{R}$ be a function defined by

$$
\beta(p)=g \circ\left(\left.\nu\right|_{X-A}\right)^{-1}(p),
$$

for $p \in \nu(X-A)$. Define a map $h: X \sqcup_{f} Y \rightarrow \mathbb{R}$ by setting

$$
h(p)=\left\{\begin{array}{ll}
\alpha(p) & \text { if } p \in \nu(Y) \\
\beta(p) & \text { if } p \in \nu(X-A)
\end{array} .\right.
$$

Readily, one has

$$
\begin{aligned}
\left.h \circ \nu\right|_{Y} & =\left.\alpha \circ \nu\right|_{Y}, \\
\left.h \circ \nu\right|_{A} & =\left.\alpha \circ \nu\right|_{A}, \\
\left.h \circ \nu\right|_{X-A} & =\left.g\right|_{X-A} .
\end{aligned}
$$

Since $g$ is a smooth extension of $\left.\alpha \circ \nu\right|_{A}$, it follows that $\left.h \circ \nu\right|_{X}=g$; therefore $h \in \mathcal{C}$. Hence $\alpha=\left.h\right|_{\nu(Y)} \in \mathcal{C}_{\nu(Y)}$.

Lemma 2.1.4 Let $\left(X \sqcup_{f} Y, \mathcal{F} \sqcup, \mathcal{G}\right)$ be the adjunction space of differential spaces $(X, \mathcal{F})$ and $(Y, \mathcal{G})$ by the smooth map $f: A \rightarrow Y$ from a closed subspace $A$ of $X$ into the differential space $(Y, \mathcal{G})$. If $\nu: X \sqcup Y \rightarrow X \sqcup_{f} Y$ is the quotient map which identifies each $a \in A$ with its image $f(a) \in Y$, then given $\mathcal{C}:=\mathcal{F} \sqcup_{f} \mathcal{G}$, a function $\alpha: \nu(Y) \rightarrow \mathbb{R}$ is in $\mathcal{C}_{\nu(Y)}$ if and only if for each $p \in \nu(Y)$, there is a neighbourhood $U_{p}$ in the underlying topology of $\mathcal{C}$ such that

$$
\left.\alpha \circ \nu\right|_{\nu^{-1}\left(U_{P}\right) \cap Y} \in \mathcal{G}_{\nu^{-1}\left(U_{P}\right) \cap Y},
$$


and

$$
\left.\alpha \circ \nu\right|_{\nu^{-1}\left(U_{p}\right) \cap A} \in \mathcal{F}_{\nu^{-1}\left(U_{p}\right) \cap A}
$$

Proof. Suppose for each $p \in \nu(Y)$, there is a neighbourhood $U_{p}$ in the topology of $\mathcal{C}$ such that conditions (2.1) and (2.2) hold. Since $\nu^{-1}\left(U_{p}\right) \cap Y$ is an open set of the topology of $\mathcal{G}$ and since $U\left\{\nu^{-1}\left(U_{p}\right) \cap Y: p \in \nu(Y)\right\}$ is an open covering of $Y$, it follows that $\left.\alpha \circ \nu\right|_{Y} \in \mathcal{G}$. Thus, by Lemma 2.1.3, $\alpha \in \mathcal{C}_{\nu(Y)}$.

Conversely, a function $\alpha: \nu(Y) \rightarrow \mathbb{R}$ is in $\mathcal{C}_{\nu(Y)}$ if and only if for each point $p \in \nu(Y)$ there is a neighbourhood $U$ of $p$ in the topology of $\mathcal{C}$ such that $\left.\alpha\right|_{U \cap \nu(Y)}=\left.g\right|_{U \cap \nu(Y)}$, for some $g \in \mathcal{C}$. It follows that

$$
\left.\alpha \circ \nu\right|_{\nu^{-1}(U \cap \nu(Y))}=\left.g \circ \nu\right|_{\nu^{-1}(U \cap \nu(Y))} .
$$

But $\nu^{-1}(U \cap \nu(Y))=\left(\nu^{-1}(U) \cap Y\right) \cup\left(\nu^{-1}(U) \cap A\right)$, with $Y \cap A=\emptyset$, so that

$$
\left.\alpha \circ \nu\right|_{\nu^{-1}(U) \cap Y}=\left.g \circ \nu\right|_{\nu^{-1}(U) \cap Y}
$$

and

$$
\left.\alpha \circ \nu\right|_{\nu^{-1}(U) \cap A}=\left.g \circ \nu\right|_{\nu^{-1}(U) \cap A}
$$

By definition of quotient structure, $g$ is a map of $\mathcal{C}$ if and only if $\left.g \circ \nu\right|_{X} \in \mathcal{F}$ and $\left.g \circ \nu\right|_{Y} \in \mathcal{G}$. Therefore,

$$
\left.\alpha \circ \nu\right|_{\nu^{-1}(U) \cap Y} \in \mathcal{G}_{\nu^{-1}(U) \cap Y}
$$


and

$$
\left.\alpha \circ \nu\right|_{\nu^{-1}(U) \cap A} \in \mathcal{F}_{\nu^{-1}(U) \cap A}
$$

Theorem 2.1.1 Let $(X, \mathcal{F})$ be a handy differential space, let $(Y, \mathcal{G})$ be a differential space, and let $\left(X \sqcup_{f} Y, \mathcal{F} \sqcup_{f} \mathcal{G}\right)$ be their adjunction space, determined by the map $f: A \rightarrow Y$, where $A$ is closed in $X$. Then the composite

$$
Y \rightarrow X \sqcup Y \rightarrow X \sqcup_{f} Y
$$

is a diffeomorphism from $Y$ to a subspace of $X \sqcup_{f} Y$.

Proof. If $\nu: X \sqcup Y \rightarrow X \sqcup_{f} Y$ is the quotient map, then obviously $\left.\nu\right|_{Y}$ is smooth and bijective. Now let us check that $\left(\left.\nu\right|_{Y}\right)^{-1}$ is also smooth. For this purpose let $\beta \in \mathcal{G} ; \beta \circ\left(\left.\nu\right|_{Y}\right)^{-1}$ is in $\mathcal{C}_{\nu(Y)}$ if and only if it satisfies the conditions given in Lemma 2.1.4. Clearly, if $U$ is any open set in $\nu(Y)$, then

$$
\left.\beta \circ\left(\left.\nu\right|_{Y}\right)^{-1} \circ \nu\right|_{\nu^{-1}(U) \cap Y}=\left.\beta\right|_{\nu^{-1}(U) \cap Y} \in \mathcal{G}_{\nu^{-1}(U) \cap Y} .
$$

On the other hand, since $\nu^{-1}(U) \cap X \subset A$, then, for all $a \in \nu^{-1}(U) \cap X$, one has $\beta \circ\left(\left.\nu\right|_{Y}\right)^{-1} \circ \nu(a)=\beta \circ f(a)$. Therefore

$$
\left.\beta \circ\left(\left.\nu\right|_{Y}\right)^{-1} \circ \nu\right|_{\nu^{-1}(U) \cap A}=\left.\beta \circ f\right|_{\nu^{-1}(U) \cap A} \in \mathcal{F}_{\nu^{-1}(U) \cap A} .
$$

The next theorem is important when it is necessary to show that the underlying topological space of an adjunction of differential spaces is indeed an adjunction of the corresponding underlying topological spaces. 
Theorem 2.1.2 Let $\left(X \sqcup_{f} Y, \mathcal{F} \sqcup_{f} \mathcal{G}\right)$ be the adjunction space of $(X, \mathcal{F})$ and $(Y, \mathcal{G})$, obtained via the attaching map $f: A \rightarrow Y$, where $A$ is closed in $X$. Then the composite

$$
X-A \rightarrow X \sqcup Y \stackrel{\nu}{\rightarrow} X \sqcup_{f} Y
$$

maps $X-A$ diffeomorphically onto an open subset of $X \cup_{f} Y$.

Proof. It is clear that $\mu:=\left.\nu\right|_{X-A}$ is smooth and bijective. To show that $\mu^{-1}$ is smooth we need to prove that for every $g \in \mathcal{F}_{X-A}$, one has $g \circ \mu^{-1} \in$ $\mathcal{C}_{\nu(X-A)}$. By definition, if $g \in \mathcal{F}_{X-A}$, then for every point $p \in X-A$, there is an open neighbourhood $U$ of $p$ contained in $X-A$ (since $X-A$ is open in $X$ ) such that $\left.g\right|_{U}=\left.h\right|_{U}$ for some $h \in \mathcal{F}$. Since the underlying topology of $\mathcal{F}$ is generated by the collection of $\alpha^{-1}(W)$, where $\alpha \in \mathcal{F}$ and $W$ is an open set of $\mathbb{R}$, by virtue of Lemma 1.1.7, we can let $U=\alpha^{-1}(0, \infty)$, with $\alpha \in \mathcal{F}$ and $\alpha(x) \geq 0$ for all $x \in X$. It is therefore obvious that $A \subset \alpha^{-1}(0)$. Suppose $\alpha(p)=k>0$; choose a nonnegative smooth increasing function $\beta: \mathbb{R} \rightarrow \mathbb{R}$ such that

$$
\beta(t)=\left\{\begin{array}{ll}
0 & \text { for } t \leq 0 \\
1 & \text { for } k-\epsilon \leq t \leq k+\epsilon
\end{array},\right.
$$

where $\epsilon>0$ is chosen in such a way that $p \in \alpha^{-1}(k-\epsilon, k+\epsilon) \subset U$. The composite $\beta \circ \alpha$ is smooth and, on another hand, $V:=\alpha^{-1}(k-\epsilon, k+\epsilon)$ is an open neighbourhood of $p$ and is such that $\left.\beta \circ \alpha\right|_{V}=1$. It follows that $\left.g\right|_{V}=\left.h \cdot(\beta \circ \alpha)\right|_{V}$. Define a smooth map $H: X \sqcup Y \rightarrow \mathbb{R}$ by setting

$$
H(x)=\left\{\begin{array}{ll}
h(x) \cdot(\beta \circ \alpha)(x) & \text { if } x \in X \\
0 & \text { if } x \in Y
\end{array} .\right.
$$


Since $(\beta \circ \alpha)(a)=0$ for all $a \in A$, it follows that $H$ is identically 0 on $A$. By its definition, $H$ induces a smooth map $\bar{H}: X \sqcup_{f} Y \rightarrow \mathbb{R}$ such that

$$
\bar{H}(\nu(x))=\left\{\begin{array}{ll}
H(x) & \text { if } x \in X \\
0 & \text { if } x \in Y
\end{array} .\right.
$$

It is evident that $\bar{H} \in \mathcal{C}$. In turn, since $V$ is an open set containing $p$, it follows that there is an open set $(a, b)$ in $\mathbb{R}$ such that $p \in H^{-1}(a, b) \subset V$. But $V$ is contained in $X-A$, so that $\nu(p) \in \bar{H}^{-1}(a, b) \subset \nu(V)$. Therefore, $\nu(V)$ is an open neighbourhood of $\nu(p)$. On another side, one has

$$
\left.\bar{H}\right|_{\nu(V)}=\left.h \circ \mu^{-1}\right|_{\nu(V)}=\left.g \circ \mu^{-1}\right|_{\nu(V)} .
$$

Thus $g \circ \mu^{-1} \in \mathcal{C}_{\nu(X-A)}$, and the proof is then done.

Theorems 2.1.1 and 2.1.2 lead to a theorem of particular importance, with the help of which we will provide another route to proving that the underlying topological space of a finite handy DW complex is a CW complex.

Theorem 2.1.3 Let $X$ be a Hausdorff handy differential space, let $A$ be a compact closed subsapce of $X$, and let $f: A \rightarrow Y$ be a smooth map from $A$ into a Hausdorff differential space $Y$. If $\mathcal{C}$ is the structure on the adjunction $X \sqcup_{f} Y$ determined by the quotient map $\nu: X \sqcup Y \rightarrow X \sqcup_{f} Y$, then every subset $U$ of $X \sqcup_{j} Y$ such that $\nu^{-1}(U)$ is open in $X \sqcup Y$ is open in the topology of $\mathcal{C}$. Thus, $X \sqcup_{f} Y$ acquires the quotient topology from $X \sqcup Y$.

Proof. First note that $\nu^{-1}(U)$ is open in $X \sqcup Y$ if and only if $\nu^{-1}(U) \cap Y$ is open in $Y$ and $\nu^{-1}(U) \cap X$ is open in $X$. Now let $p$ be a point in $U \cap \nu(Y)$; there 
is $q \in \nu^{-1}(U) \cap Y$ such that $\nu(q)=p$. Suppose that $q \in \nu^{-1}(U) \cap(Y-f(A))$. Since $\nu^{-1}(U) \cap(Y-f(A))$ is an open subset of $Y$, there is a nonnegative structure map $g_{1}: Y \rightarrow \mathbb{R}$ such that $q \in g_{1}^{-1}(0, \infty) \subset \nu^{-1}(U) \cap(Y-f(A))$. Thus, $\left.g_{1}\right|_{f(A)}=0$. In addition, let $g_{2}: X \rightarrow \mathbb{R}$ be the identically zero map, and define $h: X \sqcup Y \rightarrow \mathbb{R}$ by $h=g_{1} \sqcup g_{2}$. Define $h^{*}: X \sqcup_{f} Y \rightarrow \mathbb{R}$ such that

$$
\begin{aligned}
& \left.h^{*} \circ \nu\right|_{Y}=g_{1} \\
& \left.h^{*} \circ \nu\right|_{X}=0 ;
\end{aligned}
$$

$h^{*}$ is well defined since $\left.h^{*} \circ \nu\right|_{A}=0=\left.h^{*} \circ \nu\right|_{f(A)}$. Thus, $h^{*} \in \mathcal{C}$; and since $q \in g_{1}^{-1}(0, \infty) \subset \nu^{-1}(U) \cap Y$, it follows that $p \in\left(h^{*}\right)^{-1}(0, \infty) \subset U \cap \nu(Y)$.

Now suppose that the point $p$ is such that there is $q \in f(A)$ with $p=\nu(q)$. So $f(A) \cap\left(\nu^{-1}(U) \cap Y\right) \neq \emptyset$. Since $q \in \nu^{-1}(U) \cap Y$ and $\nu^{-1}(U) \cap Y$ is open in $Y$, there is a nonnegative smooth map $F: Y \rightarrow \mathbb{R}$ such that $q \in$ $F^{-1}(0, \infty) \subset \nu^{-1}(U) \cap Y$. It follows that $F \circ f: A \rightarrow \mathbb{R}$ is smooth and if $\bar{q}$ is a point of $A$ such that $f(\bar{q})=q$ then $\bar{q} \in(F \circ f)^{-1}(0, \infty) \subset \nu^{-1}(U) \cap A$. Let $r \in A$. There exist an open neighbourhood $U_{r} \subset X$ of $r$ and a smooth map $g_{r}: X \rightarrow \mathbb{R}$ such that $\left.F \circ f\right|_{U_{r} \cap A}=\left.g_{r}\right|_{U_{r} \cap A}$. If $g_{r}(r) \neq 0$, then $F \circ f(r) \neq 0$ and $r \in(F \circ f)^{-1}(0, \infty) \subset \nu^{-1}(U) \cap A$; one can in that case choose a smaller open neighbourhood $W_{r}$ of $r$, contained in $\nu^{-1}(U) \cap X$, such that $\left.F \circ f\right|_{W_{r} \cap A}=\left.g_{r}\right|_{W_{r} \cap A}$ and $g_{r}(s) \neq 0$ for each $s \in W_{r}$. Since $A$ is compact, one needs only finitely many $U_{r_{i}}, i=1, \ldots, n$, to cover $A$. Consider the covering $\mathcal{U}=\left\{U_{r_{1}}, \ldots, U_{r_{n}}, X-A\right\}$ of $X$ where, if $F \circ f\left(r_{l}\right) \neq 0$, then $U_{r_{l}}=W_{r_{l}}$. Let $\left\{\lambda_{j}\right\}_{j \in J}$ be a smooth partition of unity subordinate to $\mathcal{U}$. Suppose here that $\mathcal{V}=\left\{V_{j}\right\}_{j \in J}$ is a locally finite open refinement of $\mathcal{U}$ such that $\operatorname{supp}\left(\lambda_{j}\right) \subset V_{j}$ for all $j \in J$. If $V_{j} \subset W_{r_{l}}$ for some $l \in\{1, \ldots, n\}$, let 
$H_{j}: X \rightarrow \mathbb{R}$ be the smooth map $H_{j}=g_{r_{l}}$. So $\left.H_{j}\right|_{W_{r_{l}} \cap A}=\left.F \circ f\right|_{W_{r_{l}} \cap A}$. If $V_{j} \subset X-A$ or if $V_{j} \subset U_{r_{l}}$, where $U_{r_{l}}$ is a neighbourhood of $r_{l}$ such that $F \circ f\left(r_{l}\right)=0$, define $H_{j}=0$. Set $H=\Sigma_{j \in J} \lambda_{j} H_{j}$. Obviously $H$ is smooth on $X$. Let $x \in H^{-1}(0, \infty)$. Then $\lambda_{j}(x) H_{j}(x) \neq 0$ for some $j$. But $\lambda_{j}(x) \neq 0$ implies that $x \in V_{j}$. Moreover, since $H_{j}(x) \neq 0$ it follows that $V_{j}$ is contained in some $W_{r_{l}}$. But $W_{r_{l}} \subset \nu^{-1}(U) \cap X$, therefore $x \in \nu^{-1}(U) \cap X$ and hence $H^{-1}(0, \infty) \subset \nu^{-1}(U) \cap X$. Consider the smooth map $H \sqcup F: X \sqcup Y \rightarrow \mathbb{R}$; since $\left.H\right|_{A}=\left.F \circ f\right|_{A}$ there is a smooth map $h: X \sqcup_{f} Y \rightarrow \mathbb{R}$ such that $h \circ \nu=H \sqcup F$ and $p \in h^{-1}(0, \infty) \subset U$.

Now, suppose that $p$ is a point in $U \cap \nu(X-A)$ and suppose that its preimage by $\nu$ is a point $r$ in $\nu^{-1}(U) \cap(X-A)$. Since $\nu^{-1}(U) \cap(X-A)$ is open, there is a nonnegative smooth function $l \in \mathcal{F}$ such that $r \in l^{-1}(0, \infty) \subset$ $\nu^{-1}(U) \cap(X-A)$. Therefore, $A \subset l^{-1}(0)$. Define $d: X \sqcup Y \rightarrow \mathbb{R}$ by setting

$$
d(x)=\left\{\begin{array}{ll}
l(x) & \text { if } x \in X \\
0 & \text { if } x \in Y
\end{array} .\right.
$$

Since $l(x)=0$ for all $x \in A$, it follows that $d$ induces a map $\bar{d}: X \sqcup_{f} Y \rightarrow \mathbb{R}$ such that

$$
\bar{d}(\nu(x))=\left\{\begin{array}{ll}
d(x) & \text { if } x \in X \\
0 & \text { if } x \in Y
\end{array} .\right.
$$

Clearly $\bar{d} \in \mathcal{C}$ and $p \in \bar{d}^{-1}(0, \infty) \subset U \cap \nu(X-A)$.

We conclude that for all $p \in U$ there is a smooth map $h \in \mathcal{C}$ such that $p \in h^{-1}(0, \infty) \subset U$. Therefore $U$ is open for the underlying topology of $\mathcal{C}$.

Theorem 2.1.3 establishes a result whereby, given a Hausdorff handy differ- 
ential space $X$ and a Hausdorff differential space $Y$, if $A$ is a compact closed subspace of $X$ and if $X \sqcup_{f} Y$ is an adjunction in differential spaces of $X$ and $Y$ then the underlying space of $X \sqcup_{f} Y$ is an adjunction in the category of topological spaces. The reason is indeed the fact that the underlying topology of the differential space $X \sqcup_{f} Y$ is the quotient topology determined by the map $\nu$.

A case of particular importance is the case whereby $X$ is a coproduct of finitely many disjoint euclidean balls and $A$ is the coproduct of boundaries of balls of $X$. More explicitly, let $\Lambda$ be a finite indexing set, let

$$
X=\mathbf{D}:=\sqcup\left\{D_{\alpha}^{n} \mid \alpha \in \Lambda, n \in I\right\}
$$

and

$$
A=\mathbf{S}:=\sqcup\left\{S_{\alpha}^{n-1} \mid \alpha \in \Lambda, n \in I\right\}
$$

where $D_{\alpha}^{n}$ is the $\alpha$-copy of the euclidean ball $D^{n}, S_{\alpha}^{n-1}$ is the boundary of $D_{\alpha}^{n}$ and $I$ is a finite subset of $\mathbb{N}$. Let

$$
\Lambda_{n}=\left\{\alpha \in \Lambda \mid D_{\alpha}^{n} \subset X\right\}
$$

and for each $\alpha \in \Lambda_{n}$, let $\Phi_{\alpha}^{n}: S_{\alpha}^{n-1} \rightarrow Y$ be a smooth map carrying $S_{\alpha}^{n-1}$ into the Hausdorff differential space $Y$ and let $f: \mathbf{S} \rightarrow Y$ be the map $f=\sqcup_{\substack{\alpha \in \Lambda \\ n \in I}} \Phi_{\alpha}^{n}$. The space $\mathbf{D} \sqcup_{f} Y$ is called the space obtained by attaching finitely many cells $D_{\alpha}^{n}$ to $Y$.

Lemma 2.1.5 Let $\mathrm{D}^{n}=\sqcup_{\alpha \in \Lambda_{n}} D_{\alpha}^{n}$. As $\mathrm{D}^{n}$ is a handy Hausdorff differential space, then if $Y$ is a Hausdorff differential space, the space, $\mathbf{D}^{n} \sqcup_{f} Y$, obtained by attaching finitely many copies $D_{\alpha}^{n}$ of $D^{n}$ is Hausdorff. 
Proof. Note that $\mathrm{D}^{n}$, as a coproduct of handy differential spaces $D_{\alpha}^{n}, \alpha \in$ $\Lambda_{n}$, is a handy differential space by virtue of Lemma 1.2.16. In light of Theorem 2.1.3, the underlying topological space of the adjunction $\mathrm{D}^{n} \sqcup_{f} Y$ is an adjunction of underlying topological spaces of $\mathbf{D}^{n}$ and $Y$. By Proposition 2.1, page 45, in [11], since $Y$ is a Hausdorff topological space, it follows that the topological space $\mathbf{D}^{n} \sqcup_{f} Y$ is Hausdorff. Thus the adjunction taken in differential spaces is Hausdorff.

Lemma 2.1.6 Let $X$ be a $D W$ complex of dimension $n$, having finitely many cells of dimension $n$, let $\Lambda$ be the set indexing the $n$-cells, and for each $\alpha \in \Lambda$, let $D_{\alpha}^{n} \sqcup_{\Phi_{\alpha}^{n}} X^{(n-1)}$ be the adjunction obtained from $X^{(n-1)}$ by attaching a copy of the closed ball $D_{\alpha}^{n}$ by means of a smooth map $\Phi_{\alpha}^{n}: S_{\alpha}^{n-1} \rightarrow X^{(n-1)}$. Let $\mathbf{D}^{n}=\sqcup_{\alpha \in \Lambda} D_{\alpha}^{n}, \mathbf{S}^{n-1}=\sqcup_{\alpha \in \Lambda} S_{\alpha}^{n-1}$ be coproducts, taken in $\mathcal{D} \mathcal{I F F}$, and let $f: \mathbf{S}^{n-1} \rightarrow X^{(n-1)}$ be the map given by $f=\sqcup_{\alpha \in \Lambda} \Phi_{\alpha}^{n}$. Then $\mathbf{D}^{n} \sqcup_{f} X^{(n-1)}$ is a $D W$ complex, diffeomorphic to $X$.

Proof. Let $\nu: \mathrm{D}^{n} \sqcup X^{(n-1)} \rightarrow \mathrm{D}^{n} \sqcup_{f} X^{(n-1)}$ be the quotient map defining the adjunction $\mathrm{D}^{n} \sqcup_{f} X^{(n-1)}$; as $X^{(n-1)}$ is Hausdorff, it follows from Lemma 2.1.5 that the differential space $\mathbf{D}^{n} \sqcup_{f} X^{(n-1)}$ is Hausdorff.

Let $(E, \Phi)$, where $\Phi=\left\{\Phi_{e}: e \in E\right\}$, be a DW decomposition of $X$. For $k \neq n$, define $k$-cells in $\mathrm{D}^{n} \sqcup_{f} X^{(n-1)}$ by

$$
\left(\mathrm{D}^{n} \sqcup_{f} X^{(n-1)}\right)^{k}=\{\nu(e): e \text { is a } k \text {-cell in } X\},
$$

and define $n$-cells in $\mathbf{D}^{n} \sqcup_{f} X^{(n-1)}$ by

$$
\left(\mathbf{D}^{n} \sqcup_{f} X^{(n-1)}\right)^{n}=\left\{\nu\left(D_{\alpha}^{n}-S_{\alpha}^{n-1}\right): \alpha \in \Lambda\right\} .
$$


If $\left(\mathbf{D}^{n} \sqcup_{f} X^{(n-1)}\right)^{(k)}$ denotes the union of all cells in $\mathbf{D}^{n} \sqcup_{f} X^{(n-1)}$ of dimension $\leq k$, then for $k<n, \nu\left(X^{(k)}\right)=\left(\mathbf{D}^{n} \sqcup_{f} X^{(n-1)}\right)^{(k)}$. The differential structure on $\mathbf{D}^{n} \sqcup_{f} X^{n-1)}$ is the collection of functions $f: \mathbf{D}^{n} \sqcup_{f} X^{n-1)} \rightarrow \mathbb{R}$ such that $\left.f\right|_{\nu(\bar{e})}$ is smooth for every closed cell $\bar{e}$ in $X^{(n-1)}$ and $\left.f\right|_{\nu\left(D_{\alpha}^{n}\right)}$ is smooth for every $\alpha \in \Lambda$. Next, let $E^{n-1}$ be the family of all cells in $X^{(n-1)}$ and define the characteristic map of $\nu(e)$, for each $e \in E^{n-1}$, as the composite $\nu \Phi_{e}$; and for each $\alpha \in \Lambda$ define $\left.\nu\right|_{D_{\alpha}^{n}}$ as the characteristic map of the $n$-cell $\nu\left(D_{\alpha}^{n}-S_{\alpha}^{n-1}\right)$. If

$$
E^{\prime}=\left\{\nu(e): e \in E^{n-1}\right\} \cup\left\{\nu\left(D_{\alpha}^{n}-S_{\alpha}^{n-1}\right): \alpha \in \Lambda\right\}
$$

and

$$
\Phi^{\prime}=\left\{\nu \Phi_{e}: e \in E^{n-1}\right\} \cup\left\{\left.\nu\right|_{D_{\alpha}^{n}}: \alpha \in \Lambda\right\},
$$

we want to show that $\left(E^{\prime}, \Phi^{\prime}\right)$ defines a DW structure on $\mathrm{D}^{n} \sqcup_{f} X^{(n-1)}$. To this end, we verify the four axioms in the definition of DW complex.

(1d) Since $E^{n-1}$ partitions $X^{(n-1)}$ and since Theorems 2.1.1 and 2.1.2 in this context imply respectively that $X^{(n-1)}$ is diffeomorphic to $\nu\left(X^{(n-1)}\right)$ and $\mathbf{D}^{n}-\mathbf{S}^{n-1}$ is diffeomorphic to $\nu\left(\mathbf{D}^{n}-\mathbf{S}^{n-1}\right)$, it follows that $\mathbf{D}^{n} \sqcup_{f} X^{(n-1)}$ is a disjoint union of its cells.

(2d) As $X^{(n-1)}$ is diffeomorphic to $\nu\left(X^{(n-1)}\right)$ and $\mathbf{D}^{n}-\mathbf{S}^{n-1}$ is diffeomorphic to $\nu\left(\mathbf{D}^{n}-\mathbf{S}^{n-1}\right)$, every map $\nu \Phi_{e}$ is a quotient map and every map $\left.\nu\right|_{D_{\alpha}^{n}}$ is also a quotient map. Moreover, since $\left.\Phi_{e}\right|_{\check{D}^{k}}$ is a diffeomorphism, it follows that $\left.\nu \Phi_{e}\right|_{D^{k}}: \breve{D}^{k} \rightarrow \nu(e)$ is a diffeomorphism. On another side, $\mathbf{D}^{n}-\mathbf{S}^{n-1}$ being diffeomorphic to $\nu\left(\mathbf{D}^{n}-\mathbf{S}^{n-1}\right)$ implies that for each $\alpha \in \Lambda,\left.\nu\right|_{D_{\alpha}^{n}}$ : $\breve{D}_{\alpha}^{n} \rightarrow \nu\left(\breve{D}_{\alpha}^{n}\right)$ is a diffeomorphism. 
(3d) If $e$ is a $k$-cell in $E^{n-1}$, then $\overline{\nu(e)}=\nu \Phi_{e}\left(D^{k}\right)=\nu(\bar{e})$; since $\bar{e}$ is contained in a finite union of cells in $X^{(n-1)}$, it follows that $\overline{\nu(e)}$ is also contained in a finite union of cells in $\mathbf{D}^{n} \sqcup_{f} X^{(n-1)}$. Now, consider $\alpha \in \Lambda$. Since $\Phi_{\alpha}^{n}\left(S_{\alpha}^{n-1}\right)$ is compact in $X^{(n-1)}$, there is a finite DW subcomplex $Y$ in $X^{n-1}$ containing $\Phi_{\alpha}^{n}\left(S_{\alpha}^{n-1}\right)$. But $\left.\nu\right|_{X^{(n-1)}}$ is a diffeomorphism, therefore $\nu \Phi_{\alpha}^{n}\left(S_{\alpha}^{n-1}\right)$ is contained in a finite DW subcomplex $\nu(Y)$. Thus

$$
\overline{\nu\left(D_{\alpha}^{n}-\overline{\left.S_{\alpha}^{n-1}\right)}\right.}=\nu\left(D_{\alpha}^{n}-S_{\alpha}^{n-1}\right) \cup \nu \Phi_{\alpha}^{n}\left(S_{\alpha}^{n-1}\right) \subset \nu\left(D_{\alpha}^{n}-S_{\alpha}^{n-1}\right) \cup \nu(Y),
$$

and since $\nu\left(D_{\alpha}^{n}\right) \subset \overline{\nu\left(D_{\alpha}^{n}-S_{\alpha}^{n-1}\right)}$, it follows that $\nu\left(D_{\alpha}^{n}\right)$ meets only finitely many cells.

(4d) Let $\Omega$ be the differential structure on $D^{n} \sqcup_{j} X^{(n-1)}$ coinduced by the map $\nu: \mathbf{D}^{n} \sqcup X^{(n-1)} \rightarrow \mathbf{D}^{n} \sqcup_{f} X^{(n-1)}$. Suppose that $\mathcal{F}\left(D_{\alpha}^{n}\right)$ is the differential structure of the ball $D_{\alpha}^{n}, \alpha \in \Lambda$, and suppose that the differential structure on $\overline{\nu(e)}$ is given by $\nu^{*}(\mathcal{F}(\bar{e}))=\left\{g \in \mathbb{R}^{\overline{\nu(e)}}:\left.g \circ \nu\right|_{\bar{e}} \in \mathcal{F}(\bar{e})\right\}$, and that on $\nu\left(D_{\alpha}^{n}\right)$ is given by $\nu^{*}\left(\mathcal{F}\left(D_{\alpha}^{n}\right)\right)=\left\{g \in \mathbb{R}^{\nu\left(D_{\alpha}^{n}\right)}:\left.g \circ \nu\right|_{D_{\alpha}^{n}} \in \mathcal{F}\left(D_{\alpha}^{n}\right)\right\}$. Define $\mathcal{M}=\left\{\overline{\nu(e)} \rightarrow \mathrm{D}^{n} \sqcup_{f} X^{(n-1)}: e \in E^{n-1}\right\} \cup\left\{\nu\left(D_{\alpha}^{n}\right) \rightarrow \mathrm{D}^{n} \sqcup_{f} X^{(n-1)}: \alpha \in \Lambda\right\} ;$ we claim that

$$
\Omega=\operatorname{coind}\{\mathcal{M}\} \text {. }
$$

Indeed, let $F \in \Omega$; by definition of $\Omega, F \in \Omega$ if and only if $\left.F \circ \nu\right|_{X(n-1)} \in \mathcal{F}$ and $\left.F \circ \nu\right|_{D^{n}} \in \sqcup_{\alpha \in \Lambda} \mathcal{F}\left(D_{\alpha}^{n}\right)$. But $\left.F \circ \nu\right|_{X^{(n-1)}} \in \mathcal{F}$ if and only if $\left.F \circ \nu\right|_{\bar{e}} \in \mathcal{F}(\bar{e})$ for each cell $e$ in $X^{(n-1)}$, that is $\left.F\right|_{\overline{\nu(e)}} \in \nu^{*}(\mathcal{F}(\bar{e}))$ for each $e$. Likewise $\left.F \circ \nu\right|_{\mathbf{D}^{n}}$ holds if and only if $\left.F \circ \nu\right|_{D_{\alpha}^{n}} \in \mathcal{F}\left(D_{\alpha}^{n}\right)$ for each $\alpha \in \Lambda$, or if and only if $\left.F\right|_{\nu\left(D_{\alpha}^{n}\right)} \in \nu^{*}\left(\mathcal{F}\left(D_{\alpha}^{n}\right)\right)$, for each $\alpha \in \Lambda$. It follows from this argument that $F \in \Omega$ if and only if $F \in$ coind $\{\mathcal{M}\}$; thus $\Omega=\operatorname{coind}\{\mathcal{M}\}$. 
Finally, to prove that $\mathbf{D}^{n} \sqcup_{f} X^{(n-1)}$ is diffeomorphic to $X$, it will suffice to show that

$$
\pi: \mathbf{D}^{n} \sqcup X^{(n-1)} \rightarrow X,
$$

where $\pi$ equals inclusion on $X^{(n-1)}$ and the map $\Phi_{\alpha}^{n}$ on $D_{\alpha}^{n}$, is a quotient map.

Obviously, $\pi$ is surjective. Let $h: X \rightarrow \mathbb{R}$ be a structure function on $X$. Since $\left.h\right|_{\Phi_{\alpha}^{n}\left(D_{\alpha}^{n}\right)} \circ \Phi_{\alpha}^{n}$ is smooth for every $\alpha \in \Lambda$, it follows that $\left.(h \circ \pi)\right|_{D^{n}}$ is smooth. We know that $\left.(h \circ \pi)\right|_{X(n-1)}$ is smooth. Thus, $\pi$ is smooth.

Now suppose that $h: X \rightarrow \mathbb{R}$ is a function such that $h \circ \pi$ is smooth on $\mathrm{D}^{n} \sqcup X^{(n-1)}$. Then,

(1) $\left.(h \circ \pi)\right|_{X^{(n-1)}}=\left.h\right|_{X^{(n-1)}}$ is smooth.

(2) $\left.(h \circ \pi)\right|_{D_{\alpha}^{n}}=\left.h\right|_{\Phi_{\alpha}^{n}\left(D_{\alpha}^{n}\right)} \circ \Phi_{\alpha}^{n}$ is smooth on $D_{\alpha}^{n}$ for each $\alpha \in \Lambda$.

The first condition implies that $\left.h\right|_{\bar{e}}$ is smooth on $\bar{e}$, whenever $\operatorname{dim} e<n$. Since for each $c \in \Lambda, \Phi_{\alpha}^{n}$ is a quotient, the second condition implies that $\left.h\right|_{\bar{e}}$ is smooth on $\bar{e}$, where $e$ is an $n$-cell. Thus $h$ is smooth on $X$, so $\pi$ is a quotient map, as desired.

Conversely, we have 
Lemma 2.1.7 Let $Y$ be a DW complex of dimension $n-1$, let $\mathrm{D}^{n}=\cup_{\alpha \in \Lambda} D_{\alpha}^{n}$ be a finite coproduct of closed $n$-balls $D_{\alpha}^{n}$, let $\mathbf{S}^{n-1}=\sqcup_{\alpha \in \Lambda} S_{\alpha}^{n-1}$ be the coproduct of boundaries of the $n$-balls $D_{\alpha}^{n}$, and let $f: \mathbf{S}^{n-1} \rightarrow Y$ be a smooth map. Then the adjunction space $X:=\mathbf{D}^{n} \sqcup_{f} Y$ formed from $Y$ and $\mathbf{D}^{n}$ by means of $f$ is a $D W$ complex, and $Y$ is its n-1-skeleton.

Proof. Let $\nu: \mathbb{D}^{n} \sqcup Y \rightarrow X$ be the hypothesized quotient map. Now $\mathbf{D}^{n}$ is a handy Hausdorff differential space and $Y$ is Hausdorff by hypothesis; by Lemma 2.1.5 it follows that $X$ is Hausdorff.

Consider $Y$ to be a subspace of $X$; then $\nu$ equals inclusion on $Y$, and equals $f$ on $\mathbf{S}^{n-1}$. Define the open cells of $X$ to be

$$
E(X)=\{\nu(e): e \in E(Y)\} \cup\left\{\nu\left(\breve{D}_{\alpha}^{n}\right): \alpha \in \Lambda\right\}
$$

and the characteristic maps to be

$$
\Phi(X)=\left\{\nu \Phi_{e}: e \in E(Y)\right\} \cup\left\{\left.\nu\right|_{D_{\alpha}^{n}}: \alpha \in \Lambda\right\},
$$

where $E(Y)$ is the collection of cells of $Y$.

Let us prove that $(E(X), \Phi(X))$ defines a DW structure on $X$.

(1d) Clearly $X$ is a disjoint union of its cells.

(2d) It is also clear that every $\nu \Phi_{e}$ is a quotient and every $\left.\nu\right|_{D_{\alpha}^{n}}$ is a quotient. Since $Y$ is diffeomorphic to $\nu(Y)$, it follows that $\left.\nu \Phi_{e}\right|_{\check{D}^{k}}$ maps $\breve{D}^{k}$ diffeomorphically onto $\nu \Phi_{e}\left(\breve{D}^{k}\right), k<n$. Likewise, since $\mathbf{D}^{n}-\mathbf{S}^{n-1}$ is diffeomorphic to $\nu\left(\mathbf{D}^{n}-\mathbf{S}^{n-1}\right)$, it follows that $\left.\nu\right|_{\check{D}_{\alpha}^{n}}$ maps diffeomorphically $\breve{D}_{\alpha}^{n}$ onto $\nu\left(\breve{D}_{\alpha}^{n}\right)$. 
(3d) We have already noted that the map $\left.\nu\right|_{D_{\alpha}^{n}}$ maps $\breve{D}_{\alpha}^{n}$ diffeomorphically onto the set $\nu\left(\breve{D}_{\alpha}^{n}\right)$. By construction, $\left.\nu\right|_{D_{\alpha}^{n}}$ maps $S_{\alpha}^{n-1}$ into $Y$. Since $\nu\left(S_{\alpha}^{n-1}\right)$ is compact in $Y$, there is a finite DW subcomplex $Y^{\prime}$ in $Y$ containing $\nu\left(S_{\alpha}^{n-1}\right)$. But $\left.\nu\right|_{Y}$ is an identity, therefore $\nu\left(D_{\alpha}^{n}\right)$ meets only finitely many cells. Now consider a $k$-cell $e$ in $Y$. It is trivial that $\overline{\nu(e)}=\nu \Phi_{e}\left(D^{k}\right)=\nu(\bar{e})$ is contained in a finite union of cells of $\nu(Y)$. Thus condition (3d) is satisfied.

(4d) follows readily. Suppose $f: X \rightarrow \mathbb{R}$ is a smooth structure on $X$. It follows that $f \circ \nu: \mathbf{D}^{n} \sqcup Y \rightarrow \mathbb{R}$ is smooth. But $\mathbf{D}^{n} \sqcup Y$ being a disjoint union, so one has $f \circ \nu$ is smooth if and only if simultaneously $\left.(f \circ \nu)\right|_{D^{n}}$ is smooth and $\left.(f \circ \nu)\right|_{Y}$ is smooth. Note as well that $\left.(f \circ \nu)\right|_{D^{n}}$ is smooth if and only if $\left.(f \circ \nu)\right|_{D_{\alpha}^{n}}$ is smooth for each $\alpha$. But $\left.\nu\right|_{D_{\alpha}^{n}}$ is a quotient, therefore $\left.f\right|_{\nu\left(D_{\alpha}^{n}\right)}$ is smooth. Since $\nu$ is the identity on $Y,\left.f\right|_{\overline{\nu(e)}}$ is smooth for every cell $\nu(e)$.

Now let $f: X \rightarrow \mathbb{R}$ be such that $\left.f\right|_{\overline{\nu(e)}}$ is smooth for every $\nu(e)$ in $\nu(Y)$ and $\left.f\right|_{\nu\left(D_{a}^{n}\right)}$ is smooth for every $\alpha \in \Lambda$. It is clear that $f \circ \nu$ is smooth on $\mathbf{D}^{n} \sqcup Y$. Since $\nu$ is a quotient, it follows that $f$ is smooth, as desired.

Theorem 2.1.4 A differential space $X$ is a $D W$ complex with finitely many cells if and only if there is a sequence of subspaces $X_{0} \subset X_{1} \subset \ldots \subset X_{m}=X$ such that $X=\left\llcorner_{n=0}^{m} X_{n}\right.$ and the following properties hold.

(i) $X_{0}$ is a discrete space.

(ii) For each $n>0$, there is an indexing set $\Lambda_{n}$ and a family of smooth maps $\left\{\Phi_{\alpha}^{n}: S_{\alpha}^{n-1} \rightarrow X_{n-1} \mid \alpha \in \Lambda_{n}\right\}$ so that

$$
X_{n}=\mathrm{D}^{n} \sqcup_{f} X_{n-1},
$$


where $\mathrm{D}^{n}=\sqcup\left\{D_{\alpha}^{n}: \alpha \in \Lambda_{n}\right\}$ and $f=\sqcup \Phi_{\alpha}^{n}$.

(ii) If $\mathcal{F}$ is the differential structure of $X$ and $\mathcal{F}_{n}$ is the structure induced on $X_{n}$ by the inclusion $X_{n} \rightarrow X$, then a function $\sigma: X \rightarrow \mathbb{R}$ is in $\mathcal{F}$ if and only if $\left.\sigma\right|_{X_{n}} \in \mathcal{F}_{n}$ for each $n \geq 0$.

Proof. Suppose that $X$ is a DW complex with finitely many cells and $X_{n}=X^{(n)}$, the $n$-skeleton. The $n$-skeletons form an ascending sequence of subspaces $X^{(0)} \subset X^{(1)} \subset \ldots \subset X^{(m)}$ such that $X^{(m)}=X$. Since $X^{(0)}$ is a DW complex on its own and any function $\sigma: X^{(0)} \rightarrow \mathbb{R}$ belongs to $\mathcal{F}_{0}$, it follows that each subset of $X^{(0)}$ is open in $X^{(0)}$. Thus $X^{(0)}$ is discrete, and hence (i) holds. For (ii), suppose that the collection of $n$-cells of $X$ is given by $\left\{e_{\alpha} \in E: \quad \alpha \in \Lambda_{n}\right\}$; if $\Phi_{e_{\alpha}}^{n}:=\Phi_{\alpha}^{n}: D^{n} \rightarrow X$ is the characteristic map of the cell $e_{\alpha}$ then $\Phi_{\alpha}^{n}$ maps $S^{n-1}$ into $X^{(n-1)}$. A function $\sigma: X^{(n)} \rightarrow \mathbb{R}$ is smooth on $X^{(n)}$ if and only if $\left.\sigma\right|_{\bar{e}}$ is smooth on $\bar{e}$ for each cell $e$ in $X^{(n-1)}$ and $\left.\sigma\right|_{\bar{e}}$ is smooth on $\bar{e}$ for each $n$-cell $e$. If we let $\nu^{n}: \mathrm{D}^{n} \sqcup X^{(n-1)} \rightarrow X^{(n)}$ equal inclusion on $X^{(n-1)}$ and the map

$$
D_{\alpha}^{n} \stackrel{\Phi_{\alpha}^{n}}{\rightarrow} X
$$

on $D_{\alpha}^{n}$, then one can show that $X^{(n)}$ is diffeomorphic to the adjunction space formed from $X^{(n-1)}$ and $\mathbf{D}^{n}$, by means of a smooth map $f=\sqcup \Phi_{\alpha}^{n}$. Indeed, one first notes that $\nu^{n}(x)=\nu^{n}\left(\Phi_{\alpha}^{n}(x)\right)$ for all $x \in S_{\alpha}^{n-1}$. Now to assert that $X^{(n)}$ is an adjunction space, it suffices to show that $\nu^{n}$ is a quotient map. Obviously $\nu^{n}$ is smooth and surjective. Suppose that $f: X^{(n)} \rightarrow \mathbb{R}$ is smooth. Then: 
(1) $\left.f\right|_{\bar{e}}$ is smooth for all closed cell $\bar{e}$ of dimension $\leq n-1$.

(2) $f \circ \Phi_{\alpha}^{n}: D_{\alpha}^{n} \rightarrow \mathbb{R}$ is smooth for each $\alpha \in \Lambda$.

Conditions (1) and (2) imply that if $f: X^{(n)} \rightarrow \mathbb{R}$ is smooth then, its pullback $f \circ \nu^{n}: \mathbf{D}^{n} \sqcup X^{(n-1)} \rightarrow \mathbb{R}$ is smooth.

Now we need to show that every function $f: X^{(n)} \rightarrow \mathbb{R}$ such that $f \circ \nu^{n}$ : $\mathrm{D}^{n} \sqcup X^{(n-1)} \rightarrow \mathbb{R}$ is smooth is smooth on $X^{(n)}$. Indeed, $f \circ \nu^{n}$ is smooth on $\mathrm{D}^{n} \sqcup X^{(n-1)}$ if and only if $\left.f\right|_{X^{(n-1)}}$ is smooth on $X^{(n-1)}$ and $\left.f\right|_{\mathrm{D}}$ is smooth on $\mathbf{D}^{n}$. In terms of cells, we will have that $\left.f\right|_{\bar{e}}$ is smooth for every closed cell of dimension $\leq n-1$ and $f \circ \Phi_{\alpha}^{n}$ is smooth for each $\alpha \in \Lambda$. But $f \circ \Phi_{\alpha}^{n}$ is smooth if and only if $\left.f\right|_{\bar{e}}$ is smooth on $\bar{e}=\Phi_{\alpha}^{n}\left(D^{n}\right)$. So $f$ is smooth on every closed cell of dimension $\leq n$. Thus $f$ is smooth on $X^{(n)}$, and hence $X^{(n)}$ is an adjunction space.

Finally, a function $\sigma \in \mathbb{R}^{X}$ is a smooth map if and only if $\left.\sigma\right|_{\bar{e}}$ is smooth for each $e \in E$; that is, if and only if $\left.\sigma\right|_{X^{(n)}}$ is smooth for each $n \geq 0$. Thus (iii) is also satisfied. Conversely, suppose that $X$ is a differential space satisfying conditions (i), (ii) and (iii). Since $X_{0}$ is discrete, it is therefore a 0 -dimensional DW complex; thus by means of Lemma 2.1.6, each $X_{n}$ is an $n$-dimensional DW complex. If $\nu^{n}: \mathbf{D}^{n} \sqcup X_{n-1} \rightarrow \mathrm{D}^{n} \sqcup_{f} X_{n-1}=X_{n}$ is the quotient map defining the adjunction $X_{n}$, and if $\iota_{n}: X_{n} \rightarrow X$ is an inclusion map carrying $X_{n}$ into $X$, we let

$$
\left.\iota_{n} \circ \nu^{n}\right|_{D_{\alpha}^{n}}: D_{\alpha}^{n} \rightarrow \mathbf{D}^{n} \rightarrow \mathbf{D}^{n} \sqcup X_{n-1} \rightarrow \mathbf{D}^{n} \sqcup_{f} X_{n-1}=X_{n} \rightarrow X
$$

to be the characteristic map of an $n$-cell $e_{\alpha}$. Now suppose that $E_{n}$ is the 
family of cells of the DW complex $X_{n}, n \geq 0$. Define

$$
E=\cup\left\{E_{n}: n \geq 0\right\}
$$

and

$$
\Phi=\left\{\text { constant maps to } X_{0}\right\} \cup \bigcup_{n \geq 1}\left\{\left.\iota_{n} \circ \nu^{n}\right|_{D_{\alpha}^{n}}: \alpha \in \Lambda_{n}\right\}
$$

The pair $(E, \Phi)$ defines a DW structure on $X$. Indeed, it is easy to see that the required axioms for a DW complex are fulfilled:

(1d) $X=\cup\left\{E_{n}: n \geq 0\right\}$, which is a disjoint union.

(2d) Since $X=\cup_{n=0}^{m} X_{n}$ and for each $n \geq 0$, and $X_{n}$ is a DW complex, it follows that if $X^{(n)}$ denotes the collection of all cells in $E$ of dimension $\leq n$, then $X^{(n)}=X_{n}$. Thus, if $e$ is an $n$-cell in $E$ and if given $\Phi_{e}=\left.\iota_{n} \circ \nu^{n-1}\right|_{D_{a}^{n}}$, then $\Phi_{e}:\left(D^{n}, S^{n-1}\right) \rightarrow\left(e \cup X^{(n-1)}, X^{(n-1)}\right)$ is a relative diffeomorphism.

(3d) For each cell $e \in E$, there is an $n \geq 0$ such that $e \in E_{n}$. Since $X_{n}$ is a DW complex and is contained in $X$, it follows that $\bar{e}$ is contained in a finite union of cells of $X$; thus (3d) holds.

(4d) is trivial.

Theorem 2.1.4 serves as a stepping stone to proving the following theorem

Theorem 2.1.5 The underlying space of a finite $D W$ complex is a $C W$ complex.

Proof. Let $X$ be a finite DW complex. Its skeletons $X^{(k)}, k=0,1, \ldots, n$ are all closed subspaces of $X$ such that $X=\cup_{k=0}^{n} X^{(k)}$ and satisfy conditions 
(i), (ii) and (iii) of Theorem 2.1.4. Under the notation of Theorem 2.1.4, we have

$$
X=X^{(n)}=\mathrm{D}^{n} \sqcup_{f^{n}} \mathrm{D}^{n-1} \sqcup_{f^{n-1}} \mathbf{D}^{n-2} \sqcup_{f^{n-2}} \ldots \sqcup_{f^{1}} X^{(0)},
$$

where $f^{k}=\sqcup_{\alpha \in \Lambda_{k}} \Phi_{\alpha}^{k}, k=1, \ldots, n$. By Theorem 2.1.3, since every $\mathbf{D}^{k}$ is a handy differential space and $X^{(0)}$ is a Hausdorff differential space, it follows that $X$ has the quotient topology determined by

$$
\nu: \mathbf{D}^{n} \sqcup\left(\mathbf{D}^{n-1} \sqcup_{f^{n-1}} \mathbf{D}^{n-2} \sqcup_{f^{n-2}} \ldots \sqcup_{f^{1}} X^{(0)}\right) \rightarrow X .
$$

Thus the underlying space of $X$ is a CW complex. 


\section{$2.2 \quad$ FW complexes}

For easy reference, we define Frölicher spaces. Let $X$ be any set, let $\mathcal{F}$ be a collection of real-valued functions on $X$, let $\mathcal{C}$ be a collection of maps $\mathbb{R} \rightarrow X$, and let $\mathcal{M}$ be the set of smooth maps $\mathbb{R} \rightarrow \mathbb{R}$.

Definition The triple $(X, \mathcal{F}, \mathcal{C})$ is a Frölicher space provided:

(i) $f \circ c \in \mathcal{M}$ for all $f \in \mathcal{F}$ and $c \in \mathcal{C}$

(ii) $\bar{\Phi} \mathcal{C}=\{f: X \rightarrow \mathbb{R} \mid f \circ c \in \mathcal{M}$ for all $c \in \mathcal{C}\}=\mathcal{F}$

(iii) $\bar{\Gamma} \mathcal{F}=\{c: \mathbb{R} \rightarrow X \mid f \circ c \in \mathcal{M}$ for all $f \in \mathcal{F}\}=\mathcal{C}$.

See [7]. The pair $(\mathcal{F}, \mathcal{C})$ is said to be a Frölicher structure. As one may have noticed, the functions determine the curves and vice versa the curves determine the functions. The topology corresponding to the Frölicher structure $(\mathcal{F}, \mathcal{C})$ is the initial topology defined by the functions of $\mathcal{F}$. Suppose that $\left(\mathcal{F}_{1}, \mathcal{C}_{1}\right)$ and $\left(\mathcal{F}_{2}, \mathcal{C}_{2}\right)$ are two Frölicher structures on the set $X$ such that $\mathcal{C}_{1} \subset \mathcal{C}_{2}$. It follows that $\mathcal{F}_{1}=\bar{\Phi} \mathcal{C}_{1} \supset \bar{\Phi} \mathcal{C}_{2}=\mathcal{F}_{2}$. However if $\left(\mathcal{F}_{1}, \mathcal{C}_{1}\right)$ and $\left(\mathcal{F}_{2}, \mathcal{C}_{2}\right)$ were such that $\mathcal{F}_{1} \subset \mathcal{F}_{2}$, then one would have $\mathcal{C}_{1}=\bar{\Gamma} \mathcal{F}_{1} \supset \bar{\Gamma} \mathcal{F}_{2}=\mathcal{C}_{2}$. In other words, the operator $\bar{\Phi}$ (resp. $\bar{\Gamma}$ ) reverses the inclusion relation between two sets of curves (resp. functions).

In [3], it is shown that if $(X, \mathcal{F}, \mathcal{C})$ is a Frölicher space, then $(X, \mathcal{F})$ is a differential space. Thus $(X, \mathcal{F}, \mathcal{C})$ is Hausdorff if and only if the underlying differential space $(X, \mathcal{F})$ is Hausdorff. Let $(X, \mathcal{F}(X), \mathcal{C}(X))$ and $(Y, \mathcal{F}(Y), \mathcal{C}(Y))$ 
be two Frölicher spaces. A map $f:(X, \mathcal{F}(X), \mathcal{C}(X)) \rightarrow(Y, \mathcal{F}(Y), \mathcal{C}(Y))$ is a map of Frölicher spaces and is said to be (Frölicher) smooth provided $f:(X, \mathcal{F}(X)) \rightarrow(Y, \mathcal{F}(Y))$ is a map of differential spaces. Frölicher spaces as objects with smooth maps as morphisms form a category. As in [3], we will adopt the expressions $\mathcal{F} \mathcal{R} \mathcal{L}$ for the category of Frölicher spaces. Furthermore, in order to avoid ambiguity between maps in $\mathcal{F} \mathcal{R L}$ and maps in $\mathcal{D I} \mathcal{F} \mathcal{F}$, we will use prefixes " $F$ " and " $d$ " to indicate that one is dealing with Frölicher smooth maps and differential smooth maps respectively.

It is proved in [7] that the category $\mathcal{F} \mathcal{R} \mathcal{L}$ is cartesian closed. For cartesian closedness, see [12].

Definition A surjective map $q:(X, \mathcal{F}(X), \mathcal{C}(X)) \rightarrow(Y, \mathcal{F}(Y), \mathcal{C}(Y))$ of Frölicher spaces is called a quotient map provided $f \in \mathcal{F}(Y)$ if and only if $f \circ q \in \mathcal{F}(X)$. The $F$-structure $(\mathcal{F}(Y), \mathcal{C}(Y))$ is called the quotient structure on $Y$, induced by $q$.

It is easy to show that if $q:(X, \mathcal{F}(X), \mathcal{C}(X)) \rightarrow(Y, \mathcal{F}(Y), \mathcal{C}(Y))$ is a quotient map of Frölicher spaces, then, given the forgetfull functor $\mathcal{U}: \mathcal{F} \mathcal{R} \mathcal{L} \rightarrow$ $\mathcal{D I} \mathcal{F} \mathcal{F}$, clearly $q=\mathcal{U}(q):(X, \mathcal{F}(X)) \rightarrow(Y, \mathcal{F}(Y))$ is a quotient map of the associated differential spaces. Therefore quotients in $\mathcal{F} \mathcal{R} \mathcal{L}$ are the same as quotients in $\mathcal{D} \mathcal{I} \mathcal{F} \mathcal{F}$.

Lemma 2.2.1 Let $X$ be any set, and let $\mathcal{F}_{0}$ be a family of real-valued functions on $X$. Then the pair $(\mathcal{F}, \mathcal{C})$, where

$$
\mathcal{C}=\left\{c: \mathbb{R} \rightarrow X \mid f \circ c \in \mathcal{M} \text { for all } f \in \mathcal{F}_{0}\right\}=\bar{\Gamma} \mathcal{F}_{0}
$$




$$
\mathcal{F}=\{f: X \rightarrow \mathbb{R} \mid f \circ c \in \mathcal{M} \text { for all } c \in \mathcal{C}\}=\bar{\Phi} \bar{\Gamma} \mathcal{F}_{0},
$$

defines a Frölicher structure on $X$, called the Frölicher structure generated by $\mathcal{F}_{0}$ and denoted $\operatorname{gen}\left(\mathcal{F}_{0}\right)$.

Proof. See [7].

It is clear that if $(\mathcal{F}, \mathcal{C})=\operatorname{gen}\left(\mathcal{F}_{0}\right)$, then $\mathcal{F}_{0} \subset \mathcal{F}$.

Lemma 2.2.2 Let $(X, \mathcal{F}, \mathcal{C})$ be a Frölicher space and let $\mathcal{F}_{0}$ be a set of realvalued functions on $X$ such that $\mathcal{F}_{0} \subset \mathcal{F}$. Then if $\left(\mathcal{F}^{\prime}, \mathcal{C}^{\prime}\right)$ is the Frölicher structure generaied by $\mathcal{F}_{0}$, it follows that

(i) $\mathcal{C} \subset \mathcal{C}^{\prime}$

(ii) $\mathcal{F}^{\prime} \subset \mathcal{F}$.

Proof. Easy verification.

Lemma 2.2.3 Let $X$ be any set, and let $\mathcal{C}_{0}$ be a family of curves into $X$. Then the pair $(\mathcal{F}, \mathcal{C})$ where

$$
\begin{gathered}
\mathcal{F}=\left\{f: X \rightarrow \mathbb{R} \mid f \circ c \in \mathcal{M} \text { for all } c \in \mathcal{C}_{0}\right\}=\bar{\Phi} \mathcal{C}_{0} \\
\mathcal{C}=\{c: \mathbb{R} \rightarrow X \mid f \circ c \in \mathcal{M} \text { for all } f \in \mathcal{F}\}=\bar{\Gamma} \bar{\Phi} \mathcal{C}_{0},
\end{gathered}
$$

defines a Frölicher structure on $X$, called the Frölicher structure generated by $\mathcal{C}_{0}$ and denoted gen $\left(\mathcal{C}_{0}\right)$. 
Proof. See [7].

Definition Let $(X, \mathcal{F}, \mathcal{C})$ be a Frölicher space, and let $A$ be a subset of $X$. Then the Frölicher space $(A, \mathcal{F}(A), \mathcal{C}(A))$ where $(\mathcal{F}(A), \mathcal{C}(A))=\operatorname{gen}\left(\left.\mathcal{F}\right|_{A}\right)$, is said to be a Frolicher subspace of $(X, \mathcal{F}, \mathcal{C})$.

Definition Let $\left\{f_{i}:\left(X_{i}, \mathcal{F}_{i}, \mathcal{C}_{i}\right) \rightarrow X, i \in I\right\}$ be a family of set maps, where triples $\left(X_{i}, \mathcal{F}_{i}, \mathcal{C}_{i}\right)$ are Frölicher spaces, and let

$$
\mathcal{C}^{*}=\left\{f_{i} \circ c_{i}: \mathbb{R} \rightarrow X \mid c_{i} \in \mathcal{C}_{i}, i \in I\right\}
$$

The Frölicher structure generated on $X$ by $\mathcal{C}^{*}$ is called the final structure corresponding to maps $\left\{f_{i}:\left(X_{i}, \mathcal{F}_{i}, \mathcal{C}_{i}\right) \rightarrow X\right\}$ and is denoted

$$
\operatorname{fin}\left(\left\{f_{i} \mid i \in I\right\}\right) \text {. }
$$

It has as structure functions the set

$$
\cap_{i \in I}\left\{f: X \rightarrow \mathbb{R} \mid f \circ f_{i} \in \mathcal{F}_{i}\right\}
$$

See [7] for initial structures.

The FW complexes that we are about to introduce are defined pretty much like DW complexes. The "F" in "FW" stands for Frölicher and the "W" comes from "CW".

In the setting of FW complexes, the triple $\left(D^{k}, \mathcal{F}\left(D^{k}\right), \mathcal{C}\left(D^{k}\right)\right)$ will denote the unit closed $k$-ball, equipped with its Frölicher subspace structure.

Definition A $k$-cell $e$ of a Frölicher space $X$ is a diffeomorphic image of the interior of the unit closed ball $D^{k}$. 
Definition A Hausdorff Frölicher space $(X, \mathcal{F}, \mathcal{C})$ is said to be a FW complex or a Frölicher CW complex with respect to a family of cells $E$ provided:

(1f) $X=\cup\{e: e \in E\}$ (disjoint union)

(2f) For each $k$-cell $e \in E$, the smooth map $\Phi_{e}:\left(D^{k}, \mathcal{F}\left(D^{k}\right), \mathcal{C}\left(D^{k}\right)\right) \rightarrow$ $(\hat{e}, \mathcal{F}(\hat{e}), \mathcal{C}(\hat{e}))$ is a quotient and maps the subspace $\left(\breve{D}^{k}, \mathcal{F}\left(\breve{D}^{k}\right), \mathcal{C}\left(\breve{D}^{k}\right)\right)$ diffeomorphically onto the subspace $(e, \mathcal{F}(e), \mathcal{C}(e))$.

(3f) if $e$ is a $k$-cell in $X$, then $\hat{e}:=\Phi_{e}\left(D^{k}\right)$ is contained in a union of finitely many cells of dimension $\leq k$.

(4f) the Frölicher structure on $X$ is the final structure corresponding to the set $\{(\hat{e}, \mathcal{F}(\hat{\imath}), \mathcal{C}(\hat{e})) \rightarrow X: e \in E\}$ of inclusions.

By the $k$-cells of a FW complex $(X, \mathcal{F}, \mathcal{C})$, we mean the elements $e \in E$.

Lemma 2.2.4 Let $\left\{\left(X_{i}, \mathcal{F}\left(X_{i}\right), \mathcal{C}\left(X_{i}\right)\right) ; i \in I\right\}$ be a family of Frölicher spaces and let $\left\{f_{i}: X_{i} \rightarrow X\right\}_{i \in I}$ be a family of set maps. Suppose that $\mathcal{F}^{F}(X)$ is the set of functions for the final Frölicher structure induced by the $f_{i}$, and suppose on the other hand that $\mathcal{F}^{d}(X)$ is the coinduced differential structure corresponding to $\left\{f_{i}:\left(X_{i}, \mathcal{F}\left(X_{i}\right)\right) \rightarrow X\right\}_{i \in I}$. Then,

$$
\mathcal{F}^{F}(X)=\mathcal{F}^{d}(X)
$$


Proof. According to [15],

$$
\mathcal{F}^{d}(X)=\left\{f: X \rightarrow \mathbb{R} \mid f \circ f_{i} \in \mathcal{F}\left(X_{i}\right) \text { for all } i \in I\right\},
$$

which is exactly the set $\mathcal{F}^{F}(X)$ of structure functions for the final Frölicher structure corresponding to the functions $f_{i}$.

It is equally interesting to check whether the partial dual of Lemma 2.2.4 holds.

Lemma 2.2.5 Let $(Y, \mathcal{F}(Y), \mathcal{C}(Y))$ be a Frölicher space. Suppose that $\mathcal{F}^{F}(X)$ is the set of functions for the initial Frölicher structure induced by the set map $\Psi: X \rightarrow Y$ and suppose that $\mathcal{F}^{d}(X)$ is the differential structure induced by $\Psi: X \rightarrow(Y, \mathcal{F}(Y))$. Then

$$
\mathcal{F}^{d}(X) \subset \mathcal{F}^{F}(X)
$$

Proof. Note first that the initial Frölicher structure induced on $X$ by $\Psi$ is given by

$$
\mathcal{C}(X)=\{c: \mathbb{R} \rightarrow X \mid f \circ \Psi \circ c \in \mathcal{M} \text { for all } f \in \mathcal{F}(Y)\}
$$

and

$$
\mathcal{F}^{F}(X)=\{f: X \rightarrow \mathbb{R} \mid f \circ c \in \mathcal{M} \text { for all } c \in \mathcal{C}(X)\}
$$

Note as well that if

$$
\mathcal{F}^{*}=\{f \circ \Psi: f \in \mathcal{F}(Y)\}
$$


then

$$
\mathcal{F}^{d}(X)=\operatorname{gen}\left(\left\{\mathcal{F}^{*}\right\}\right)
$$

Let $f \in \mathcal{F}^{d}(X)$ and let $p \in X$ be any point. Then there exist $f^{1}, \ldots, f^{n} \in$ $\mathcal{F}(Y)$ such that

$$
\left.f\right|_{U_{p}}=\left.\omega \circ\left(f^{1} \circ \Psi, \ldots, f^{n} \circ \Psi\right)\right|_{U_{p}}
$$

where $U_{p}$ is a neighbourhood of $p$ for the underlying topology of $\mathcal{F}^{*}$, and where $\omega \in C^{\infty}\left(\mathbb{R}^{n}\right)$. Thus

$$
\left.f \circ c\right|_{c^{-1}\left(U_{p}\right)}=\left.\omega \circ\left(f^{1} \circ \Psi \circ c, \ldots, f^{n} \circ \Psi \circ c\right)\right|_{c^{-1}\left(U_{p}\right)}
$$

for all $c \in \mathcal{C}(X)$. It is easy to see that $\omega \circ\left(f^{1} \circ \Psi \circ c, \ldots, f^{n} \circ \Psi \circ c\right): \mathbb{R} \rightarrow \mathbb{R}$ is smooth. Since $\left\{c^{-1}\left(U_{p}\right): p \in X, c \in \mathcal{C}(X)\right\}$ is an open covering of $\mathbb{R}$, it follows from (2.3) that $f \circ c: \mathbb{R} \rightarrow \mathbb{R}$ is smooth for all $c \in \mathcal{C}(X)$. Hence $f \in \mathcal{F}^{F}(X)$, and so $\mathcal{F}^{d}(X) \subset \mathcal{F}^{F}(X)$.

The reverse inclusion $\mathcal{F}^{F}(X) \subset \mathcal{F}^{d}(X)$ does not always hold. As a matter of fact, consider the set $\mathbb{Q}$ of rationals. If $\iota_{\mathbb{Q}}: \mathbb{Q} \rightarrow \mathbb{R}$ is an inclusion map and $\mathbb{R}:=(\mathbb{R}, \mathcal{F}(\mathbb{R}), \mathcal{C}(\mathbb{R}))$ is the usual real Frölicher space, then the initial Frölicher structure corresponding to $\iota_{\mathbb{Q}}$ is defined by

$$
\mathcal{C}(\mathbb{Q})=\mathbb{Q} \quad \text { and } \quad \mathcal{F}^{F}(\mathbb{Q})=\mathbb{R}^{\mathbb{Q}}
$$

Whereas

$$
\mathcal{F}^{d}(\mathbb{Q})=\operatorname{gen}\left(\left\{\left.\mathcal{F}(\mathbb{R})\right|_{\mathbb{Q}}\right\}\right)
$$

It is clear that $\mathcal{F}^{d}(\mathbb{Q})$ is strictly contained in $\mathcal{F}^{F}(\mathbb{Q})$. 
We let $\mathbb{R}_{+, F}$ denote the set $\mathbb{R}_{+}=\{r \in \mathbb{R} \mid r \geq 0\}$ endowed with its Frölicher subspace structure as a subset of $\mathbb{R}$. Similarly $\mathbb{R}_{+, d}$ will denote the set $\mathbb{R}_{+}$, equipped with its differential structure as a subset of $\mathbb{R}$. It is shown in [2] that $\mathcal{U}\left(\mathbb{R}_{+, F}\right)=\mathbb{R}_{+, d}$, where $\mathcal{U}: \mathcal{F} \mathcal{R} \mathcal{L} \rightarrow \mathcal{D} \mathcal{I F} \mathcal{F}$ is the forgetful functor. This means that $\mathbb{R}_{+, F}$, as a Frölicher space, has the same functions that it has as a differential space. We also let $\mathbb{R}_{F}$ denote the real line, viewed as a Frölicher space, and $\mathbb{R}_{d}$ denote the real line, viewed as a differential space. However instead of using the too cumbersome notation $\mathbb{R}_{F} \times_{F} \mathbb{R}_{+, F}$ to denote the product of $\mathbb{R}_{F}$ with $\mathbb{R}_{+, F}$ in $\mathcal{F} \mathcal{R} \mathcal{L}$, we will use the notation $\mathbb{R} \times_{F} \mathbb{R}_{+}$rather. Similarly $\mathbb{R} \times_{d} \mathbb{R}_{+}$will denote the product of $\mathbb{R}_{d}$ with $\mathbb{R}_{+, d}$ in $\mathcal{D} \mathcal{I} \mathcal{F} \mathcal{F}$.

Let $J$ be an infinite indexing set. Consider a topology on $\mathbb{R}^{J}$ having for basis the family $\mathcal{B}$ of open sets of the form $U=x_{i \in J} U_{j}$, where, for finitely many $j, U_{j}=\left(a_{j}-\varepsilon_{j}, a_{j}+\varepsilon_{j}\right)$ with $a_{j}, \varepsilon_{j} \in \mathbb{R}$ and $\varepsilon_{j}>0$, and $U_{j}=\mathbb{R}$ otherwise.

Definition [4] A function $f: \mathbb{R}^{J} \rightarrow \mathbb{R}$ depends locally at $p \in \mathbb{R}^{J}$ on finitely many variables if there is a basic open set $U \in \mathcal{B}$ containing $p$ and a finite number of indices $j_{1}, \ldots, j_{m} \in J$ such that

$$
\left.f\right|_{U}\left(\left(x_{j}\right)_{j \in J}\right)=g\left(x_{j_{1}}, \ldots, x_{j_{m}}\right)
$$

for some smooth function

$$
g:\left\{\left(x_{j_{1}}, \ldots, x_{j_{m}}\right) \mid\left(x_{j}\right)_{j \in J} \in U\right\} \rightarrow \mathbb{R}
$$

In addition to the above definition, we will need the two following lemmas on infinite products in categories $\mathcal{F} \mathcal{R} \mathcal{L}$ and $\mathcal{D} \mathcal{I F} \mathcal{F}$, which have been lucidly proved in [4]. 
Lemma 2.2.6 Let $J$ be an infinite set. Every $F$-smooth function $f: \mathbb{R}^{J} \rightarrow$ $\mathbb{R}$ is $d$-smooth if and only if, given any point $p \in \mathbb{R}^{J}, f$ depends locally at $p$ on finitely many variables. (The topology on $\mathbb{R}^{J}$ is the topology generated by the basis $\mathcal{B}$.)

Lemma 2.2.7 Let $J$ be an infinite set. The countable product $\mathbb{R}^{J}$ has, as a differential space, the same functions that it has as a Frölicher space.

Now, let us show

Lemma 2.2.8 Let $J$ be a countable set. The product $\mathbb{R}^{J} \times \mathbb{R}_{+}$has, as a differential space, the same structure functions that it has as a Frölicher space.

Proof. Lemma 2.2.7 says that the structure functions for the product Frölicher structure on $\mathbb{R}^{J+1}:=\mathbb{R}_{F}^{J+1}$ are the same as the structure functions for the product differential structure defined on $\mathbb{R}^{J+1}:=\mathbb{R}_{d}^{J+1}$. So the differential space $\mathbb{R}^{J} \times_{d} \mathbb{R}$ is diffeomorphic to the underlying differential space of the Frölicher space $\mathbb{R}^{J} \times_{F} \mathbb{R}$. ( We use notations $\mathbb{R}^{J} \times_{d} \mathbb{R}$ and $\mathbb{R}^{J} \times_{F} \mathbb{R}$ in lieu of $\mathbb{R}_{d}^{J+1}$ and $\mathbb{R}_{F}^{J+1}$ respectively, because soon we will compare structure functions on $\mathbb{R}^{J} \times_{d} \mathbb{R}_{+}$to those on $\mathbb{R}^{J} \times_{F} \mathbb{R}_{+}$.) Let $\eta: \mathbb{R}_{F} \rightarrow \mathbb{R}_{+, F}$ be a function defined by setting $\eta(x)=x^{2}$. It is shown in [2] that $\eta$ is a quotient map in $\mathcal{F} \mathcal{R} \mathcal{L}$. Since in cartesian closed categories such as $\mathcal{F} \mathcal{R} \mathcal{L}$ the product of an identity map with a quotient is again a quotient, it follows that the 
map

$$
1_{\mathbb{R}^{J}} \times \eta: \mathbb{R}^{J} \times_{F} \mathbb{R} \rightarrow \mathbb{R}^{J} \times_{F} \mathbb{R}_{+}
$$

is a quotient in $\mathcal{F} \mathcal{R} \mathcal{L}$. Since the diagram

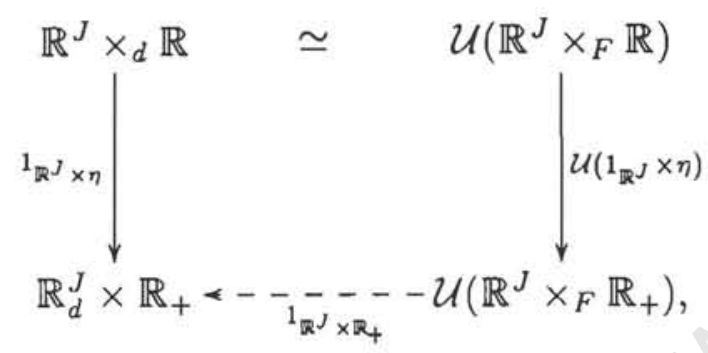

where $\mathcal{U}: \mathcal{F} \mathcal{R} \mathcal{L} \rightarrow \mathcal{D I} \mathcal{F} \mathcal{F}$ is the forgetful functor, commutes, it follows that $\mathcal{U}\left(\mathbb{R}^{J} \times_{F} \mathbb{R}_{+}\right)$has a finer structure than $\mathbb{R}^{J} \times_{d} \mathbb{R}_{+}$.

Let $f: \mathbb{R}^{J} \times_{F} \mathbb{R}_{+} \rightarrow \mathbb{R}$ be $F$-smooth map and $p=\left(\left(p_{j}\right)_{j \in J}, a\right) \in \mathbb{R}^{J} \times_{F} \mathbb{R}_{+}$. Let $a=0$. Since the $F$-smooth map $f \circ(1 \times \eta): \mathbb{R}^{J} \times_{F} \mathbb{R} \rightarrow \mathbb{R}$ is, by Lemma 2.2.7, $d$-smooth on $\mathbb{R}^{J} \times_{d} \mathbb{R}$, Lemma 2.2.6 says there is an open set $V \in \mathcal{B}$ of $p$ in $\mathbb{R}^{J} \times \mathbb{R}$ such that $f \circ(1 \times \eta)$ depends on only finitely many coordinates on $V$. The open set may be of the form

$$
V=\times_{j \in J} U_{j} \times(-\sqrt{b}, \sqrt{b}),
$$

where for finitely many $j, U_{j}$ is an arbitrarily small neighbourhood of $p_{j}$ and where $b>0$ is taken arbitrarily small. Now, let $W=(1 \times \eta)(V)$. It is clear that $W=\times_{j \in J} U_{j} \times[0, b)$, and $f$ depends on only finitely many coordinates on $W$.

Let $a>0$ and $W^{\prime}=\times_{j \in J} U_{j} \times(b, c)$ with $0<b<a<c$. Then

$$
V^{\prime}=(1 \times \eta)^{-1}\left(W^{\prime}\right)=\times_{j \in J} U_{j} \times((-\sqrt{c},-\sqrt{b}) \cup(\sqrt{b}, \sqrt{c})) .
$$


As above, $f \circ(1 \times \eta)$ depends on only finitely many coordinates on an open neighbourhood $\times_{j \in J} U_{j} \times(-\sqrt{c},-\sqrt{b})$ of $\left(\left(p_{j}\right)_{j \in J},-\sqrt{a}\right)$ (resp. on an open neighbourhood $\times_{j \in J} U_{j} \times(\sqrt{b}, \sqrt{c})$ of $\left(\left(p_{j}\right)_{j \in J}, \sqrt{a}\right)$. It follows then that $f$ depends on only finitely many coordinates on an open neighbourhood $\times_{j \in J} U_{j} \times(b, c)$ of each $p$. Thus, by Lemma 2.2.6, $f$ is $d$-smooth on a neighbourhood of $p$. Hence, the proof is complete.

Let $J$ be finite. The set $H=\mathbb{R}^{J} \times \mathbb{R}_{+}$is a half plane in $\mathbb{R}^{J+1}$. Let $X$ be a smooth manifold with boundary. The question of smoothness at boundary points for a map $f: X \rightarrow \mathbb{R}$ is decided in terms of the notion of smoothness for maps $g: H \rightarrow \mathbb{R}$. Since the notion of smooth functions for $\mathbb{R}^{J} \times_{d} \mathbb{R}_{+} \rightarrow \mathbb{R}$ and $\mathbb{R}^{J} \times_{F} \mathbb{R}_{+} \rightarrow \mathbb{R}$ are the same, the notion of smooth map $f: X \rightarrow \mathbb{R}$ from the standpoint of Frölicher spaces is the same as that from the standpoint of differential spaces. We thus have:

Corollary 2.2.1 The unit ball $D^{k}, k \in \mathbb{N}$, as a submanifold of $\mathbb{R}^{k}$ from the differential space or Frölicher space point of view has the same structure functions when viewed as a differential or Frölicher subspace of $\mathbb{R}^{k}$.

Lemma 2.2.9 Let $(X, \mathcal{F}(X), \mathcal{C}(X))$ be a $F W$ complex, and let $Y$ be a Frölicher subspace of $X$ made up of cells $e$ of $X$ such that if $e$ is a cell in $Y$ then $\hat{e}$ is also in $Y$. Assume that $\Phi_{e}^{X}$ (resp. $\left.\Phi_{e}^{Y}\right)$ is the characteristic map associated to the cell e of $X$ (resp. Y) and assume furthermore that, for each cell e of $X$ contained in $Y$, one has $\Phi_{e}^{X}=\Phi_{e}^{Y}$. Then, $Y$ is a FW complex on its own right. 
Proof. That (1f), (2f) and (3f) are verified is easy to check. Denote by $E(Y)$ the collection of cells of $Y$. It remains to show that $Y$ has the Frölicher structure corresponding to the inclusions $\hat{e} \rightarrow Y, e \in E(Y)$.

Let $(\mathcal{F}(Y), \mathcal{C}(Y))$ be the Frölicher structure induced by the inclusion $\iota_{Y}$ : $Y \rightarrow(X, \mathcal{F}(X), \mathcal{C}(X))$. Let

$$
\mathcal{F}^{*}=\left\{\left.f\right|_{Y}: f \in \mathcal{F}(X)\right\}
$$

Then

$$
\mathcal{C}(Y)=\left\{c: \mathbb{R} \rightarrow Y \mid \iota_{Y} \circ c \in \mathcal{C}(X)\right\}=\bar{\Gamma} \mathcal{F}^{*}
$$

Clearly, if $g \in \mathcal{F}^{*}$, then every $\left.g\right|_{\hat{e}}$ is smooth. Therefore, if

$$
\mathcal{G}=\left\{f: Y \rightarrow \mathbb{R}|f|_{\hat{e}} \in \mathcal{F}(\hat{e}) \text { for all } e \in E(Y)\right\}
$$

then $\mathcal{F}^{*} \subset \mathcal{G}$. Since $\mathcal{G}$ is the set of functions for the Frölicher structure induced by the inclusions $(\hat{e}, \mathcal{F}(\hat{e}), \mathcal{C}(\hat{e})) \rightarrow Y$, it follows, by Lemma 2.2.2, that $\mathcal{F}(Y) \subset \mathcal{G}$.

Using the fact that the unit closed ball $D^{k}$, as a differential subspace of $\left(\mathbb{R}^{k}, \mathcal{F}\left(\mathbb{R}^{k}\right)\right)$, has the same functions that it has as a Frölicher subspace of $\left(\mathbb{R}^{k}, \mathcal{F}\left(\mathbb{R}^{k}\right), \mathcal{C}\left(\mathbb{R}^{k}\right)\right)$ and since quotients in $\mathcal{F} \mathcal{R} \mathcal{L}$ are the same as quotients in $\mathcal{D I F F}$, one can show, by the same techniques used in in the proof of Theorem 1.2 .1 , that every map $f \in \mathcal{G}$ can be extended smoothly to the entire space $X$. Thus, one writes $f=\left.\hat{f}\right|_{Y}$ for some Frölicher smooth $\hat{f} \in \mathcal{F}(X)$, and hence $\left.\mathcal{G} \subset \mathcal{F}(X)\right|_{Y} \subset \mathcal{F}(Y)$. 
Definition A FW subcomplex $(Y, E(Y))$ of a FW complex $(X, E(X))$ is a FW complex such that $Y$ is a Frölicher subspace of $X, E(Y) \subset E(X)$ and $\Phi_{e}^{Y}=\Phi_{e}^{X}$ for every cell $e$ in $Y$.

Every $k$-skeleton of a FW complex is a FW subcomplex.

The following lemmas can be proved exactly as in case of DW complexes.

Lemma 2.2.10 If $X$ is a FW complex, and $e$ is any cell in $X$, then $\hat{e}$ is contained in a finite $F W$ subcomplex.

Lemma 2.2.11 If $X$ is a FW complex and if $Y$ and $Z$ are $F W$ subcomplexes, then $Y \cap Z$ and $Y \cup Z$ are $F W$ subcomplexes of $X$.

Since for each $k \in \mathbb{N}, d$-functions on $D^{k}$ are the same as $F$-functions, and since cells for either DW complexes or FW complexes are diffeomorphic images of open balls, one might wonder whether every DW complex is an underlying space of some FW complex and vice versa every FW complex has a DW complex as an underlying topological space. If the latter is so, then the underlying topological space of a FW complex is a CW complex.

Theorem 2.2.1 If $(X, \mathcal{F})$ is a $D W$ complex, with cells $\{e: e \in E\}$ and characteristic maps $\left\{\Phi_{e}: e \in E\right\}$, and is the image of some Frölicher space $(X, \mathcal{F}, \mathcal{C})$ under the forgetful functor $\mathcal{U}: \mathcal{F} \mathcal{R} \mathcal{L} \rightarrow \mathcal{D} \mathcal{I} \mathcal{F} \mathcal{F}$, then the Frölicher space $(X, \mathcal{F}, \mathcal{C})$, under the same cell decomposition and with the same characteristic maps, is a FW complex. 
Proof. Let $e$ be a cell in $X$, let $\Phi_{e}^{d}=\Phi_{e}$ be the characteristic map of $e$, when $e$ is viewed as a cell of the differential space $(X, \mathcal{F})$, and let $\Phi_{e}^{F}$ be the characteristic map of the cell $e$, when $e$ is viewed as a cell of the Frölicher space $(X, \mathcal{F}, \mathcal{C})$. By hypothesis, $\Phi_{e}^{F}=\Phi_{e}^{d}=" \Phi_{e}$. Suppose that $\operatorname{dim} e=k$. Since $(X, \mathcal{F})$ and $(X, \mathcal{F}, \mathcal{C})$ have the same topology, it follows that $\Phi_{e}^{F}\left(D^{k}\right)=\bar{e}=\Phi_{e}^{d}\left(D^{k}\right)$. But $(X, \mathcal{F})$ is a DW complex, therefore $\bar{e}$ is contained in a finite union of cells of dimension $\leq k$. Thus, (3f) holds.

Let $\mathcal{F}(\bar{e})$ be the differential structure of the closed cell $\bar{e}$. Then $\mathcal{F}(\bar{e})$ is the family of functions $f: \bar{e} \rightarrow \mathbb{R}$ such that $f \circ \Phi_{e}$ is a structure function for the usual differential structure $\mathcal{F}^{d}\left(D^{k(e)}\right)$ of $D^{k(e)}$, where $k(e)$ is the dimension of $e$. Since Corollary 2.2.1 says that for every $D^{n}$ the d-smooth maps and the F-smooth maps coincide, and since quotients in $\mathcal{F} \mathcal{R} \mathcal{L}$ are the same as quotients in $\mathcal{D} \mathcal{I F F}$, it follows that the Frollicher structure induced by $\sqcup_{e \in E} \Phi_{e}: \sqcup_{e \in E}\left(D^{k(e)}, \mathcal{F}\left(D^{k(e)}\right), \mathcal{C}\left(D^{k(e)}\right)\right) \rightarrow X$ on $X$ is the pair $(\mathcal{F}, \bar{\Gamma} \mathcal{F})$, where $\bar{\Gamma} F=C$. Thus, axiom (4f) is satisfied.

Now, let us examine axiom (2f). Since by Boman's theorem [10], d-smooth maps and F-smooth maps are the same on smooth manifolds without boundary, it follows that $\operatorname{int} D^{k}, k \in \mathbb{N}$, as a submanifold of $\mathbb{R}^{k}$, has, as a differential space, the same structure functions that it has as a Frölicher space. For the purpose of making our notation simpler and clearer, we put

$$
\operatorname{int} D^{k}=\breve{D}^{k}
$$

So if $\left(\breve{D}^{k}, \mathcal{F}^{d}\left(\breve{D}^{k}\right)\right)$ is the differential subspace induced by the inclusion $\breve{D}^{k} \rightarrow$ $\left(\mathbb{R}^{k}, \mathcal{F}\left(\mathbb{R}^{k}\right)\right)$ and $\left(\breve{D}^{k}, \mathcal{F}^{F}\left(\breve{D}^{k}, \mathcal{C}\left(\breve{D}^{k}\right)\right)\right.$ is the Frölicher subspace induced by 
the inclusion $\breve{D}^{k} \rightarrow\left(\mathbb{R}^{k}, \mathcal{F}\left(\mathbb{R}^{k}\right), \mathcal{C}\left(\mathbb{R}^{k}\right)\right)$, then $\mathcal{F}^{d}\left(\breve{D}^{k}\right)=\mathcal{F}^{F}\left(\breve{D}^{k}\right)$. Because of this equality, we will use the notation $\mathcal{F}\left(\breve{D}^{k}\right)$ to denote both $\mathcal{F}^{d}\left(\breve{D}^{k}\right)$ and $\mathcal{F}^{F}\left(\breve{D}^{k}\right)$. Denote by $\mathcal{F}(e)$ the differential structure induced on $e$ by the inclusion $e \rightarrow(\bar{e}, \mathcal{F}(\bar{e}))$. Since $\left.\Phi_{e}\right|_{\breve{D}^{k}}:\left(\breve{D}^{k}, \mathcal{F}\left(\breve{D}^{k}\right)\right) \rightarrow(e, \mathcal{F}(e))$ is a diffeomorphism, it follows that $\left.\Phi_{e}\right|_{\breve{D}^{k}}:\left(\breve{D}^{k}, \mathcal{F}\left(\breve{D}^{k}\right), \mathcal{C}\left(\breve{D}^{k}\right)\right) \rightarrow(e, \mathcal{F}(e), \bar{\Gamma} \mathcal{F}(e))$ is a diffeomorphism of Frölicher spaces. But the Frölicher structure induced on $e$ by the inclusion $e \rightarrow(\bar{e}, \mathcal{F}(\bar{e}), \bar{\Gamma} \mathcal{F}(\bar{e}))$ is the structure generated by the set $\left.\mathcal{F}(\bar{e})\right|_{e}$. By Lemma $2.2 .5,\left.\mathcal{F}(\bar{e})\right|_{e} \subset \mathcal{F}(e) \subset \mathcal{F}^{F}(e)$, where $\left(\mathcal{F}^{F}(e), \mathcal{C}^{F}(e)\right)$ is the F-structure induced on $e$ by the inclusion $e \rightarrow(\bar{e}, \mathcal{F}(\bar{e}), \bar{\Gamma} \mathcal{F}(\bar{e}))$. But $(\mathcal{F}(e), \bar{\Gamma} \mathcal{F}(e))$ is a Frölicher structure whose $\mathcal{F}(e)$ is the smallest set of functions containing $\left.\mathcal{F}(\bar{e})\right|_{e}$. Therefore,

$$
\mathcal{F}(e)=\mathcal{F}^{F}(e) \quad \text { and } \quad \bar{\Gamma} \mathcal{F}(e)=\mathcal{C}^{F}(e) .
$$

Hence, the Frölicher structure on $\breve{D}^{k}$ induced by the inclusion

$$
\breve{D}^{k} \rightarrow\left(D^{k}, \mathcal{F}\left(D^{k}\right), \mathcal{C}\left(D^{k}\right)\right)
$$

is diffeomorphic to the Frölicher structure on $e$, induced by the inclusion $e \rightarrow(\bar{e}, \mathcal{F}(\bar{e}), \bar{\Gamma} \mathcal{F}(\bar{e}))$. Therefore, (2f) holds, and the proof is thence complete.

It is known that, given a Frölicher space $(X, \mathcal{F}, \mathcal{C})$, the pair $(X, \mathcal{F})$, that one gets under the forgetful functor $\mathcal{U}: \mathcal{F} \mathcal{R} \mathcal{L} \rightarrow \mathcal{D I F} \mathcal{F}$, is a differential space. Now, suppose that the Frölicher space $(X, \mathcal{F}, \mathcal{C})$ is a FW complex. It would be interesting to show whether the differential space $(X, \mathcal{F})$ is a $\mathrm{DW}$ complex. In fact, it is so. We prove this result in the next theorem.

Theorem 2.2.2 Let $(X, \mathcal{F}, \mathcal{C})$ be a $F W$ complex, with $E$ as the collection 
of cells and $\Phi=\left\{\Phi_{e}: e \in E\right\}$ the collection of characteristic maps. Then the differential space $(X, \mathcal{F})$ is a $D W$ complex under the same cell decomposition $E$ and with the same characteristic maps.

Proof. It is obvious that (1d) is satisfied. Let $k \in \mathbb{N}$. Let $\mathcal{F}^{F}\left(D^{k}\right)$ be the set of functions for the Frölicher structure induced by the inclusion $D^{k} \rightarrow\left(\mathbb{R}^{k}, \mathcal{F}\left(\mathbb{R}^{k}\right), \mathcal{C}\left(\mathbb{R}^{k}\right)\right)$, and let $\mathcal{F}^{d}\left(D^{k}\right)$ be the differential structure induced by the inclusion $D^{k} \rightarrow\left(\mathbb{R}^{k}, \mathcal{F}\left(\mathbb{R}^{k}\right)\right)$. By Corollary 2.2.1, we know that $\mathcal{F}^{F}\left(D^{k}\right)=\mathcal{F}^{d}\left(D^{k}\right)$. Let $k(e)$ denote the dimension of the cell $e \in E$. Since $\sqcup_{e \in E} D^{k(e)}$ has, as a coproduct in $\mathcal{F} \mathcal{R} \mathcal{L}$, the same functions that it has as a coproduct in $\mathcal{D} \mathcal{I F} \mathcal{F}$, and since quotients in $\mathcal{F} \mathcal{R} \mathcal{L}$ are the same as quotients in $\mathcal{D I F F}$, it follows that

$$
\sqcup_{e \in E} \Phi_{e}: \sqcup_{e \in E} D^{k(e)} \rightarrow X
$$

defines the same quotient structure in $\mathcal{F R \mathcal { L }}$ as in $\mathcal{D I F F}$. Thus, axiom (4d) is satisfied. Axiom (3d) is also satisfied since (3f) holds.

Now, let us show that (2d) holds. Let $e$ be a $k$-cell of the FW complex $(X, \mathcal{F}, \mathcal{C})$ and $\Phi_{e}$ be its characteristic map. We need to show that the interior $\breve{D}^{k}$ of $D^{k}$ has as a differential space the same functions that it has as a Frölicher space. This property is met simply because of the fact that $\breve{D}^{k}$ is a smooth manifold without boundary. Now call $\mathcal{F}^{d}(e)$ the differential structure induced on $e$ by the inclusion $e \rightarrow(\hat{e}, \mathcal{F}(\hat{e}))$ and call $\left(\mathcal{F}^{F}(e), \mathcal{C}(e)\right)$ the Frölicher structure induced on $e$ by the inclusion $e \rightarrow\left(\hat{e}, \mathcal{F}^{F}(\hat{e}), \mathcal{C}(\hat{e})\right)$. By Lemma 2.2.5, $\mathcal{F}^{d}(e) \subset \mathcal{F}^{F}(e)$. Since $(X, \mathcal{F}, \mathcal{C})$ is a FW complex

$$
\left.\Phi_{e}\right|_{\breve{D}^{k}}:\left(\breve{D}^{k}, \mathcal{F}\left(\breve{D}^{k}\right), \mathcal{C}\left(\breve{D}^{k}\right)\right) \rightarrow\left(e, \mathcal{F}^{F}(e), \mathcal{C}(e)\right)
$$


is a diffeomorphism of Frölicher spaces. It follows that

$$
\left.\Phi_{e}\right|_{\breve{D}^{k}}:\left(\breve{D}^{k}, \mathcal{F}\left(\breve{D}^{k}\right)\right) \rightarrow\left(e, \mathcal{F}^{F}(e)\right)
$$

is a diffeomorphism of differential spaces. On another hand, as $\mathcal{F}^{d}\left(\breve{D}^{k}\right)=$ $\mathcal{F}^{F}\left(\breve{D}^{k}\right)$, it follows from Theorem 2.2.1 that

$$
\left.\Phi_{e}\right|_{\breve{D}^{k}}:\left(\breve{D}^{k}, \mathcal{F}\left(\breve{D}^{k}\right)\right) \rightarrow\left(e, \mathcal{F}^{d}(e)\right)
$$

is a diffeomorphism. Thus, $\mathcal{F}^{d}(e)=\mathcal{F}^{F}(e)$, and hence (2d) is met.

From Theorems 2.2 .1 and 2.2.2, we have

Theorem 2.2.3 The Frölicher space $(X, \mathcal{F}, \mathcal{C})$ has the structure of a $F W$ complex if and only if the differential space $(X, \mathcal{F})$ has the structure of a $D W$ complex.

Since every FW complex is a DW complex, one has

Corollary 2.2.2 If $X$ is a FW complex and $e$ is a $k$-cell in $X$, then

$$
\hat{e}=\bar{e}
$$

where $\hat{e}=\Phi_{e}\left(D^{k}\right)$ and $\bar{e}$ denotes the closure of $e$ with respect to the topology induced by the structure functions.

It is also possible to view a FW complex as a space built up from a collection of closed balls, equipped with their Frölicher structures as subspaces of real 
euclidean Frölicher spaces, by forming, as for CW complexes and DW complexes, appropriate quotient spaces. The fact that quotients in $\mathcal{F} \mathcal{R} \mathcal{L}$ are the same as quotients in $\mathcal{D} \mathcal{I F \mathcal { F }}$ gives us (almost) for free, so to speak, many nice properties pertaining to DW complexes. But first, we need to adapt the machinery used in building up a DW complex from its cells to the present situation.

Definition Let $\mathcal{U}=\left\{U_{i}: i \in I\right\}$ be an open covering of a Frölicher space $X$. Then a Frölicher smooth partition or simply a smooth partition of unity subordinate to $\mathcal{U}$ is a collection $\left\{f_{j}\right\}_{j \in J}$ of $F$-smooth maps $f_{j}: X \rightarrow[0,1]$ such that:

(i) There is a locally finite open refinement $\left\{V_{j}: j \in J\right\}$ of $\mathcal{U}$ such that $\operatorname{supp}\left(f_{j}\right) \subset V_{j}$ for all $j \in J$,

(ii) $\sum_{j} f_{j}(x)=1$ for each $x \in X$.

Now, let $A$ be a subspace of a Frölicher space $X$. Then, $A$ is said to have the global extension property if every $F$-smooth map $f: A \rightarrow \mathbb{R}$ can be extended to some $F$-smooth map $\bar{f}: X \rightarrow \mathbb{R}$. Unlike closed subspaces of a differential space with smooth partitions of unity, no proof has been yet found to ascertain whether closed subspaces of a Frölicher space have the global extension property. Since the global extension property is so essential in adjoining cells, we define a handy Frölicher space to be a Frölicher space $X$ satisfying the following points:

(1) every open covering of $X$ has a smooth partition of unity. 
(2) Every closed subspace of $X$ has the global extension property.

Definition Let $X$ be a handy Frölicher space, and let $f: A \rightarrow Y$ be a smooth map carrying a non-empty closed subspace $A$ of $X$ into a Frölicher space $Y$. The adjunction space $X \sqcup_{f} Y$ of $X$ and $Y$ determined by $f$ is the quotient space obtained from the coproduct $X \sqcup Y$ by identifying each set

$$
\{y\} \cup f^{-1}(y)
$$

for all $y \in Y$, to a point.

By Boman's theorem [10], we know that, given a sphere $S^{k-1}$, it has, as a differential subspace of $\mathbb{R}^{k}$ the same structure functions it has as a Frölicher subspace of $\mathbb{R}^{k}$. Because the closed ball $D^{k}$ has the same structure functions for both differential subspace structure and Frölicher subspace structure inherited as a subspace of $\mathbb{R}^{k}$, and because quotients in $\mathcal{F} \mathcal{R} \mathcal{L}$ and $\mathcal{D} \mathcal{I} \mathcal{F} \mathcal{F}$ are the same, and finally because every real-valued smooth function on $S^{k}$ can be extended to some real-valued smooth function on $D^{k}$, one has

Theorem 2.2.4 Let $Y$ be a Hausdorff Frölicher space and let $f: S^{k-1} \rightarrow Y$ be a smooth map. If $\mathcal{C}$ is the structure on the adjunction $D^{k} \cup_{f, F} Y$ determined by the quotient map $\nu: D^{k} \sqcup Y \rightarrow D^{k} \sqcup_{f, F} Y$, then every subset $U$ of $D^{k} \sqcup_{f, F} Y$ such that $\nu^{-1}\left(C^{r}\right)$ is open in $D^{k} \cup Y$ is open in the topology of $\mathcal{C}$. Thus the topology on $D^{k} \cup_{f, F} Y$ is the quotient topology determined by $\nu$.

Proof. This is a particular case of Theorem 2.1.3. 
One more thing that we would like to say on this alternative way of describing FW complexes is that proofs of Lemma 2.1.6, Lemma 2.1.7 and Theorem 2.1.4 can be carried over word for word, except that one will have to replace "differential space" by "Frölicher space" wherever it appears.

After showing that the underlying topological space of a DW complex is a CW complex, one wonders whether cellular homology of DW complexes can be developed in parallel with that of CW complexes. Unfortunately we don't have time to expound on this topic.

Finally a few words can be said about singular homology theory of differential spaces. An exposition of homology theory of topological spaces can be found in Rotman [18], or in almost any other standard textbook on introduction to algebraic topology.

Definition The differential standard $n$-simplex in $\mathbb{R}^{n+1}$ is the differential subspace

$$
\Delta^{n}=\left\{\left(x_{1}, x_{2}, \ldots, x_{n+1}\right) \in \mathbb{R}^{n+1}: \text { each } x_{i} \geq 0 \text { and } \sum x_{i}=1\right\} ;
$$

we shall denote ts structure by $\varepsilon^{n}\left(\Delta^{n}\right)$ or $\varepsilon\left(\Delta^{n}\right)$.

If $\left\{e_{0}, \ldots, e_{n}\right\}$ is the set of vertices of $\Delta^{n}$, then the $i$-th face is the differential subspace

$$
\begin{aligned}
F_{i}\left(\Delta^{n}\right)= & \left\{p \in \Delta^{n}: p=t_{0} e_{0}+\ldots+t_{i-1} e_{i-1}+t_{i+1} e_{i+1}+\ldots+t_{n} e_{n}\right. \\
& \text { with } \left.t_{0}+\ldots+t_{i-1}+t_{i+1}+\ldots+t_{n}=1 \text { and each } t_{i} \geq 0\right\} .
\end{aligned}
$$


Definition Let $X$ be a differential space. A differential singular $n$-simplex, or a differential $n$-simplex, or simply an $n$-simplex, is a smooth map $\sigma$ : $\Delta^{n} \rightarrow X$, where $\Delta^{n}$ is the differential standard $n$-simplex.

Thus a differential 0 -simplex is just a point in $X$, and a differential 1-simplex is a curve in $X$, called a path in $X$.

Definition Let $X$ be a differential space. The free abelian group generated by the differential $n$-simplices of $X$ is denoted $S_{n}(X)$ and called the differential singular chain group of $X$. The elements of $S_{n}(X)$ are called $n$-chains of $X$.

Definition Let $X$ be a differential space. The boundary homomorphism of order $n$ is the map $\partial_{n}: S_{n}(X) \rightarrow S_{n-1}(X)$ such that

$$
\partial_{n} \sigma=\sum_{i=0}^{n}(-i)^{i} \sigma \varepsilon_{i}^{n}, \quad \text { for all } \sigma \in S_{n}(X)
$$

where $\varepsilon_{i}^{n}: \Delta^{n-1} \rightarrow \Delta^{n}$ is an affine map taking $\Delta^{n-1}$ onto $F_{i}\left(\Delta^{n}\right)$, the $i$-th face of $\Delta^{n}$.

The map $\varepsilon_{i}^{n}$ is called the $i$-th face map and sends vertices $\left\{e_{0}, \ldots, e_{n-1}\right\}$ of $\Delta^{n-1}$ to vertices $\left\{e_{0}, \ldots, \hat{e}_{i}, \ldots, e_{n}\right\}$ of $\Delta^{n}$ such that to every point $p=$ $\left(t_{0}, \ldots, t_{n-1}\right)$, one assigns

$$
\begin{aligned}
\varepsilon_{i}^{n}:\left(t_{0}, \ldots, t_{n-1}\right) & \mapsto\left(t_{0}, \ldots, t_{i-1}, 0, t_{i}, \ldots, t_{n}\right) \quad \text { if } i \geq 1 \\
\varepsilon_{0}^{n}:\left(t_{0}, \ldots, t_{n-1}\right) & \mapsto\left(0, t_{0}, \ldots, t_{n-1}\right) .
\end{aligned}
$$

( The hat "means "omitted".) Since affine maps are smooth, it follows that $\partial_{n} \sigma$ is indeed an $(n-1)$-chain; we call $\partial_{n} \sigma$ the boundary of $\sigma$. 
The collection of chain groups $S_{n}(X)$, of a differential space $X$, and boundary homomorphisms $\partial_{n}$ can be organized into the sequence

$$
\ldots \rightarrow S_{n}(X) \stackrel{\partial_{n}}{\rightarrow} S_{n-1}(X) \rightarrow \ldots \rightarrow S_{1}(X) \stackrel{\partial_{9}}{\rightarrow} S_{0}(X) \stackrel{\partial_{9}}{\rightarrow} 0
$$

and one shows as for topological spaces that $\partial_{n} \partial_{n+1}=0$, that is boundaries have no boundaries. So, for each $n \geq 0, \operatorname{Ker} \partial_{n} \supset \operatorname{Im} \partial_{n+1}$. The sequence (2.4) is called a chain complex of $X$; we will denote it by $S_{*}(X)$. If $f: X \rightarrow Y$ is a map of differential spaces and $\sigma$ is a singular $n$-simplex in $X$, then the composite $f_{\#}(\sigma)=f \circ \sigma$ is an $n$-simplex in $Y$. This correspondence in turn defines a homomorphism

$$
f_{\#}: S_{n}(X) \rightarrow S_{n}(Y) \quad \text { for each } n \text {. }
$$

Lemma 2.2.12 If $f: X \rightarrow Y$ is a smooth map, then one has an infinite commutative diagram

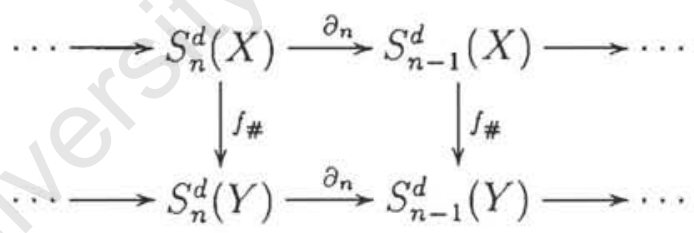

Corollary 2.2.3 Let $A$ be a subspace of a differential space $X$. Then the inclusion map $i: A \rightarrow X$ induces a monomorphism $i_{\#}: S_{n}^{d}(A) \rightarrow S_{n}^{d}(X)$ for each $n \geq 0$.

Proof. Define $i_{\#}: S_{n}^{d}(A) \rightarrow S_{n}^{d}(X)$ by setting $i_{\#}(\sigma)=i \circ \sigma$ and extending by linearity to the whole of $S_{n}^{d}(A)$. Clearly if $\sigma, \tau$ are $n$-simplices in $A$, then 
$i_{\#}(\sigma)=i_{\#}(\tau)$ implies that $\sigma=\tau$. Thus $i_{\#}$ is a monomorphism on simplices and, as $S_{n}^{d}(A)$ is the free group generated by simplices, on $S_{n}^{d}(A)$.

Definition For each $n \geq 0$, the quotient group $\operatorname{Ker} \partial_{n} / \operatorname{Im} \partial_{n+1}$ is called the $n$-dimensional homology group of $X$ and is denoted $H_{n}(X)$. We will write $Z_{n}(X)$ and $B_{n}(X)$ for $\operatorname{Ker} \partial_{n}$ and $\operatorname{Im} \partial_{n}$ respectively; the elements of $Z_{n}(X)$ are called $n$-cycles, and those of $B_{n}(X) n$-boundaries.

We will now check whether the dimension and homotopy axioms hold in the category of differential spaces. First, let $X$ be a differential space. For $x, y \in X$, we say $x \mathcal{R} y$ if there is a smooth map $\sigma:[0,1] \rightarrow X$ such that $\sigma(0)=x$ and $\sigma(l)=y$. It is routine to check that $\mathcal{R}$ is indeed an equivalence relation. The smooth map $\sigma$ is called a path in $X$ from $x$ to $y$; the equivalence classes of this relation are called path components of $X$. Finally, the space $X$ is said to be path connected if for every $x, y \in X$, there is a path in $X$ from $x$ to $y$.

Theorem 2.2.5 (Dimension axiom). If $X$ is a one-point differential space, then $H_{n}(X)=0$ for all $n>0$.

Proof. The proof is the same as for a one-point topological space.

We list the following results without proof, since they can be derived as in the context of topological spaces: 
(i) If $X$ is a space and $\left\{X_{\lambda}: \lambda \in \Lambda\right\}$ are the path components of $X$, then for every $n$

$$
H_{n}(X) \cong \sum_{\lambda} H_{n}\left(X_{\lambda}\right)
$$

(Note that if $\left(g_{\lambda}\right) \in \sum_{\lambda} H_{n}\left(X_{\lambda}\right)$ then $g_{\lambda}=0$ for all but finitely many $\lambda$.)

(ii) If $X$ is a nonempty path connected space, then $H_{0}(X)=\mathbb{Z}$. Moreover, if $x_{0}, x_{1} \in X$, then $\overline{x_{0}}=\overline{x_{1}}\left(\overline{x_{0}}\right.$ : equivalence class of $\left.x_{0}\right)$ is a generator of $H_{0}(X)$.

Our next aim has been to show that the homotopy axiom in homology theory of topological spaces holds in this setting as well. The technique in Rotman [18] can almost be applied verbatim to prove that if $f, g: X \rightarrow Y$ are differentially homotopic then $H_{n}(f)=H_{n}(g)$, for all $n \geq 0$. But, first let us define what we mean by differentially homotopic maps.

Definition Let $I=[0,1]$ have the differential subspace structure inherited as a subspace of $\mathbb{R}$. Two smooth maps $f, g: X \rightarrow Y$ of differential spaces are said to be differentially homotopic if there is a smooth map $F: X \times I \rightarrow Y$ such that

$$
F(x, 0)=f(x) \text { and } F(x, 1)=g(x)
$$

for all $x \in X$. Such a map $F$ is called a homotopy.

For a pair of maps $f, g: X \rightarrow Y$ the notation $f \simeq g$ will signify that maps $f$ and $g$ are differentially homotopic. Intuitively speaking, $f \simeq g$ if and only if $f$ can be "smoothly transformed" into $g$. The relation $\simeq$ is an equivalence 
relation on $\operatorname{Hom}_{d}(X, Y)$, where $\operatorname{Hom}_{d}(X, Y)$ is the collection of all smooth maps from $X$ into $Y$.

Theorem 2.2.6 If $X$ is a convex differential subspace of euclidean differential space, then

$$
H_{n}(X)=0 \quad \text { for } n \geq 1 \text {. }
$$

Proof. Let $\sigma: \Delta^{n} \rightarrow X$ be an $n$-simplex and $b$ be an arbitrary point in $X$. Define an $(n+1)$-simplex $(b . \sigma): \Delta^{n+1} \rightarrow X$ such that $(b . \sigma)\left(t_{0}, t_{1}, \ldots, i_{n+1}\right)=\left\{\begin{array}{ll}b & \text { for } t_{0}=1 \\ \alpha\left(t_{0}\right) b+\left(1-\alpha\left(t_{0}\right)\right) \sigma\left(\frac{t_{1}}{1-t_{0}}, \ldots, \frac{t_{n+1}}{1-t_{0}}\right) & \text { for } t \neq 1\end{array}\right.$, where $\alpha:[0,1] \rightarrow[0,1]$ is a smooth map, in the sense of differential spaces, defined by

$$
\alpha(t)=\left\{\begin{array}{l}
1 \text { for } 1-\epsilon \leq t \leq 1 \text { with } \epsilon>0 \\
0 \text { for } t=0
\end{array} .\right.
$$

One readily sees, with $\epsilon>0$ suitably small, that $b . \sigma$ is well defined and smooth, thus an $(n+1)$-simplex in $X$.

As in [18], define $c_{n}(\sigma): S_{n}^{d}(X) \rightarrow S_{n+1}^{d}(X)$ by setting $c_{n}(\sigma)=b . \sigma$ and extending by linearity. To show that

$$
\partial_{n+1} c_{n}(\sigma)=\sigma-c_{n-1} \partial_{n}(\sigma)
$$

the technique in [18] applies word for word, and leads to the conclusion that $Z_{n}(X)=B_{n}(X)$, hence $H_{n}(X)=0$ for all $n>0$.

Our version of the homotopy axiom reads: 
Theorem 2.2.7 (Homotopy axiom) If $f, g: X \rightarrow Y$ are differentially homotopic, then

$$
H_{n}(f)=H_{n}(g) \quad \text { for all } n \geq 0 \text {. }
$$

Let $A$ be a subspace of a differential space $X$, and let $i: A \rightarrow X$ denote the inclusion map. Since $i_{\#}: S_{n}^{d}(A) \rightarrow S_{n}^{d}(X)$ is a monomorphism, one can consider $S_{n}^{d}(A)$ to be a subgroup of $S_{n}^{d}(X)$. The quotient group $S_{n}^{d}(X) / S_{n}^{d}(A)$, denoted by $S_{n}^{d}(X, A)$, is called the group of relative differential $n$-chains ( $\bmod$ $A$ ) or group of differential $n$-chains of the pair $(X, A)$. The homomrphism $\bar{\partial}_{n}: S_{n}^{d}(X, A) \rightarrow S_{n-1}^{d}(X, A)$, given by

$$
\bar{\partial}_{n}\left(\sigma+S_{n}^{d}(A)\right)=\partial_{n} \sigma+S_{n-1}^{d}(A)
$$

where $\partial_{n}$ is the toundary operator $S_{n}^{d}(X) \rightarrow S_{n-1}^{d}(X)$, is called the boundary operator for relative homology.

Definition The group of relative differential $n$-cycles $\bmod A$ is

$$
Z_{n}^{d}(X, A)=\left\{\sigma \in S_{n}^{d}(X, A) \mid \bar{\partial}_{n} \sigma=0\right\}
$$

The group of relative differential $n$-boundaries $\bmod A$ is

$$
B_{n}^{d}(X, A)=\left\{\sigma \in S_{n}^{d}(X, A) \mid \bar{\partial}_{n+1} \gamma=\sigma \text { for some } \gamma \in S_{n+1}^{d}(X, A)\right\}
$$

There are some difficulties with the developement here, but we had no time to consider them. 


\section{Bibliography}

[1] G.E. BREDON, TOPOLOGY AND GEOMETRY, Springer Verlag, Berlin, 1993.

[2] P. CHERENACK, Applications of Frölicher Spaces to Cosmology, Annales Univ. Sci. Budapest, 41 (1998), 63-91.

[3] P. CHERENACK, Frölicher versus Differential Spaces: A Prelude to Cosmology, Papers in Honour of B. Banaschewski, pp 391-413, Kluwer Academic Publishers. Netherlands 2000.

[4] P. CHERENACK, P. MULTARZYNSKI, Smooth Exponential Objects and Smooth Distributions, to appear.

[5] G.E. COOKE, R.L. FINNEY, HOMOTOPY OF CELL COMPLEXES, Princeton University Press, Princeton, 1967.

[6] A. FOMENKO, DIFFERENTIAL GEOMETRY AND TOPOLOGY, (Translated from Russian by D. Leites) Consultants Bureau. New York and London, 1987 
[7] A. FRÖLICHER, A. KRIEGL, LINEAR SPACES AND DIFFERENTIATION THEORY, Wiley- Interscience, New York, 1971

[8] M. HELLER, P. MLLTARZYNSKI, W.SASIN, The Algebraic Approach to Space-Tmie Geometry, Acta Cosmologica Fasciculus XVI (1989).

[9] M.W. HIRSCH, DIFFERENTIAL TOPOLOGY, Springer-Verlag, 1976.

[10] A. KRIEGL, P. MICHOR, THE SETTING OF GLOBAL ANALYSIS, American Mathematical Society, Providence, R.I., 1997.

[11] A. LUNDELL, S. WEINGRAM, THE TOPOLOGY OF CW COMPLEXES, Van Nostrand Reinhold Company, New York, 1969.

[12] S. MAC LANE, CATEGORIES FOR THE WORKING MATHEMATICIAN, Springer Verlag, Berlin, 1971.

[13] W. MASSEY, SINGULAR HOMOLOGY THEORY, Springer Verlag, Berlin, 1980.

[14] C.R.F. MAUNDER, ALGEBRAIC TOPOLOGY, Van Nostrand Reinhold Company, London, 1970.

[15] P. MULTARZYNSKI, P.CHERENACK, P. NTUMBA, On Differential Structures for Cartesian Products, Far East J. Math. Sci. (FJMS), Special Volume (1999), Part III, (Geometry and Topology), 373-396.

[16] P. MULTARZYNSKI, Z. PASTERNAK-WINIARSKI, Differential Groups and Their Lie Algebras, Demonstartio Mathematica, Vol. XXIV n0 3-4, 1991. 
[17] J. MUNKRES, ELEMENTS OF ALGEBRAIC TOPOLOGY, The Benjamin/ Cummings Publishing Company, Inc. California, 1984.

[18] J.J. ROTMAN, AN INTRODUCTION TO ALGEBRAIC TOPOLOGY, Springer, Berlin, 1988.

[19] W. SASIN, THE WEDGE SUM OF DIFFERENTIAL SPACES, Proceedings of the Winter School on Geometry and Physics, SRNI, 6-13 January, 1990.

[20] W. SASIN, GEOMETRICAL PROPERTIES OF GLUING OF DIFFERENTIAL SPACES, Demonstratio Mathematica, Vol. XXIV n0 3-4 1991.

[21] W. SASIN, K. SPALLEK, GLUING OF DIFFERENTIABLE SPACES AND APPLICATIONS, Math. Ann. 292, 85-102 (1992).

[22] W. SASIN, Gluing of Differential Spaces, Demonstratio Mathematica, Vol XXV N0 1-2, 1992.

[23] R. SIKORSKI, Differential Modules, Colloq. Math., 24 (1971), 45-79.

[24] J.H.C. WHITEHEAD, Combinatorial Homotopy I, Bull. Amer. Math. Soc. 55 (1949), 213-245. 